\title{
COMPACTIFICATIONS OF MODULI OF POINTS AND LINES IN THE PROJECTIVE PLANE
}

\author{
LUCA SCHAFFLER AND JENIA TEVELEV
}

\begin{abstract}
Projective duality identifies the moduli spaces $\mathbf{B}_{n}$ and $\mathbf{X}(3, n)$ parametrizing linearly general configurations of $n$ points in $\mathbb{P}^{2}$ and $n$ lines in the dual $\mathbb{P}^{2}$, respectively. The space $\mathbf{X}(3, n)$ admits Kapranov's Chow quotient compactification $\overline{\mathbf{X}}(3, n)$, studied also by Lafforgue, Hacking, Keel, Tevelev, and Alexeev, which gives an example of a KSBA moduli space of stable surfaces: it carries a family of certain reducible degenerations of $\mathbb{P}^{2}$ with $n$ "broken lines". Gerritzen and Piwek proposed a dual perspective, a compact moduli space parametrizing certain reducible degenerations of $\mathbb{P}^{2}$ with $n$ smooth points. We investigate the relation between these approaches, answering a question of Kapranov from 2003.
\end{abstract}

\section{INTRODUCTION}

Projective duality associates to a configuration of $n$ points in $\mathbb{P}^{2}$ a configuration of $n$ lines in the dual $\mathbb{P}^{2}$, and conversely. Our motivating question is: how does projective duality between points and lines in the plane behave in families and under degenerations? More precisely, denote by $\mathbf{B}_{n}$ and $\mathbf{X}(3, n)$ the moduli spaces of $n$ general points in $\mathbb{P}^{2}$ and $n$ general lines in the dual $\left(\mathbb{P}^{2}\right)^{\vee}$ respectively. Projective duality gives an identification

$$
\mathbf{B}_{n}=\mathbf{X}(3, n),
$$

but these moduli spaces carry distinct $\mathbb{P}^{2}$-bundles, one with $n$ points (sections) and another with $n$ lines (divisors). The problem is to find their modular compactifications, i.e. to extend the $\mathbb{P}^{2}$-bundles to universal families over the compactifications. For $\mathbf{X}(3, n)$, a modular compactification is given by Kapranov's Chow quotient $\overline{\mathbf{X}}(3, n)$ Kap93a, which carries a family of reducible degenerations of $\mathbb{P}^{2}$ with $n$ "broken lines". This is an example of the moduli space of stable pairs (varieties with divisors) of Kollár, Shepherd-Barron, and Alexeev [HKT06, Ale15]. There are no comparable moduli spaces for varieties with marked points.

We start by studying the Gerritzen-Piwek compactification $\overline{\mathbf{B}}_{n}$. Although defined differently in [GP91], $\overline{\mathbf{B}}_{n}$ is isomorphic to the Zariski closure of the image of the map

$$
\mathbf{B}_{n} \hookrightarrow \prod_{\mathcal{Q}_{n}} \mathbb{P}^{1},
$$

where $\mathcal{Q}_{n}$ is the set of ordered quintuples of distinct labels $v_{1}, \ldots, v_{5} \in\{1, \ldots, n\}$. Every map $\mathbf{B}_{n} \rightarrow \mathbb{P}^{1}$ sends $\left(p_{1}, \ldots, p_{n}\right)$ to the cross-ratio of four points obtained by projecting $p_{v_{1}}, \ldots, p_{v_{4}}$ from $p_{v_{5}} \in \mathbb{P}^{2}$. The analogous construction for points in $\mathbb{P}^{1}$ instead of $\mathbb{P}^{2}$ yields

2020 Mathematics Subject Classification. 14J10, 14D06, 52C35, 52B40, 51E24, 14 T90.

Key words and phrases. Moduli space, line arrangement, compactification, matroid, Mustafin join. 
$\overline{\mathrm{M}}_{0, n} \cong \overline{\mathrm{X}}(2, n)$ [HKT09, Theorem 9.18]. It follows from Luxton's [Lux08, Proposition 3.2.8] (see the proof of Theorem 1.2) that a similar result holds for lines in $\mathbb{P}^{2}$ up to normalization:

Theorem 1.1. There exists a finite birational morphism $\overline{\mathbf{X}}(3, n) \rightarrow \overline{\mathbf{B}}_{n}$. In particular, we have $\overline{\mathbf{X}}(3, n)^{\nu} \cong \overline{\mathbf{B}}_{n}^{\nu}$. (For a reduced scheme $X$, we denote by $X^{\nu}$ its normalization.)

For $n=6$, Luxton proves that in fact $\overline{\mathbf{X}}(3,6) \cong \overline{\mathbf{B}}_{6}$, [Lux08, Theorem 4.1.15]. We prove a very general result in combinatorial matroid theory (see \$2) and combine it with the theory of Lafforgue's varieties $\bar{\Omega}^{Q}$ ([Laf03], see $\$ 3$ ), which generalize Kapranov's construction of $\overline{\mathbf{X}}(3, n)$ to arbitrary matroids, to prove perhaps the most general result in this direction.

Theorem 1.2. Let $\bar{\Omega}^{Q}$ be the Lafforgue variety corresponding to a matroid polytope $Q$ of rank $r$ and dimension $n-1$ such that $r \geq 3$, or $r=2$ and $n \geq 5$. The product of face maps

$$
\bar{\Omega}^{Q} \rightarrow \prod_{F \in \mathcal{F}} \bar{\Omega}^{F}=\prod_{F \in \mathcal{F}} \mathbb{P}^{1}
$$

is a finite morphism and its restriction to the main stratum of $\bar{\Omega}^{Q}$ is birational onto its image if the matroid is framed. Here $\mathcal{F}$ is the collection of faces of $Q$ in the boundary of the hypersimplex $\Delta(r, n)$ that are equivalent to $\Delta(2,4)$.

Next we investigate the claim from GP91 that $\overline{\mathbf{B}}_{n}$ is a modular compactification which parametrizes certain degenerations of $\mathbb{P}^{2}$ with configurations of $n$ points. As common in moduli theory, to construct the family over a compactification, the first step is to identify potential central fibers in one-parameter degenerations. Formally, if $\mathbb{k}$ is an algebraically closed field, $R=\mathbb{k}[[t]]$, and $K=\mathbb{k}((t))$ is the field of fractions of $R$, then given $\operatorname{Spec}(K) \rightarrow$ $\mathbf{B}_{n}$, we need to construct a family over $\operatorname{Spec}(R)$. We recall the definition of Mustafin join Mus78.

Definition 1.3. For a free $R$-submodule $L \subseteq K^{3}$ of rank 3, let $\mathbb{P}(L)=\operatorname{Proj}\left(\operatorname{Sym}\left(L^{\vee}\right)\right)$. The submodule $L$ is called a lattice. Given a finite set of lattices $\Sigma=\left\{L_{1}, \ldots, L_{m}\right\}$, the corresponding Mustafin join $\mathbb{P}(\Sigma)$ is the Zariski closure of the diagonal embedding

$$
\mathbb{P}_{K}^{2} \hookrightarrow \mathbb{P}\left(L_{1}\right) \times_{R} \ldots \times_{R} \mathbb{P}\left(L_{m}\right) .
$$

We denote by $\mathbb{P}(\Sigma)_{\mathbb{k}}$ the central fiber of $\mathbb{P}(\Sigma)$, a degeneration of $\mathbb{P}_{K}^{2}$. The Bruhat-Tits building $\mathfrak{B}_{3}^{0}$ is the set of equivalence classes of lattices modulo rescaling by $\lambda \in K^{*}$. The Mustafin join $\mathbb{P}(\Sigma)$ only depends on the equivalence classes of lattices $\left[L_{1}\right], \ldots,\left[L_{m}\right] \in \mathfrak{B}_{3}^{0}$.

Mustafin joins were used to determine special fibers of various families of compactified moduli spaces. One of the first examples is [GP91, where Gerritzen and Piwek constructed a Mustafin join associated to a one-parameter degeneration of $n$ points in $\mathbb{P}^{2}$ (see below). Kapranov showed in [Kap93b] that an analogous construction for $n$ points in $\mathbb{P}^{1}$ gives all fibers of the universal family over $\overline{\mathrm{M}}_{0, n}$. The interpretation of the family of visible contours over $\overline{\mathbf{X}}(3, n)$ in terms of Mustafin joins was obtained in [KT06]. This work was based on [Fal01, Laf03], where Mustafin joins were also studied. In [CS10, CHSW11], it was shown that the central fibers of arbitrary Mustafin joins are reduced and Cohen-Macaulay. 
Definition $1.4\left(\right.$ GP91]). Let $a_{1}, \ldots, a_{n} \in \mathbb{P}^{2}(K)$ be points in general linear position. We call $\mathbf{a}=\left(a_{1}, \ldots, a_{n}\right)$ an arc. Given a lattice $L$, the valuative criterion of properness gives unique extensions $\bar{a}_{1}^{L}, \ldots, \bar{a}_{n}^{L} \in \mathbb{P}(L)(R)$, which are sections of the $\mathbb{P}^{2}$-bundle $\mathbb{P}(L) \rightarrow$ Spec $(R)$. We say that $L$ is a stable lattice with respect to an arc $\mathbf{a}$ if at least four of the limits $\bar{a}_{1}^{L}(0), \ldots, \bar{a}_{n}^{L}(0)$ in the central fiber $\mathbb{P}(L)_{\mathbb{k}} \subseteq \mathbb{P}(L)$ are in general linear position. Let

$$
\Sigma_{\mathbf{a}} \subseteq \mathfrak{B}_{3}^{0}
$$

be the set of stable lattice classes with respect to a. This is a finite set [KT06, Lemma 5.19]. We call $\mathbb{P}\left(\Sigma_{\mathbf{a}}\right)$ the Mustafin join for an arc to distinguish it from arbitrary Mustafin joins. The valuative criterion of properness gives $n$ sections $\bar{a}_{1}, \ldots, \bar{a}_{n}$ of $\mathbb{P}\left(\Sigma_{\mathbf{a}}\right) \rightarrow \operatorname{Spec}(R)$.

By Theorem 6.9, the Mustafin join $\mathbb{P}\left(\Sigma_{\mathbf{a}}\right) \rightarrow \operatorname{Spec}(R)$ for an arc is smooth along $n$ disjoint sections $\bar{a}_{1}, \ldots, \bar{a}_{n}$. The central fiber $\mathbb{P}\left(\Sigma_{\mathbf{a}}\right)_{\mathbb{k}}$ is reduced and Cohen-Macaulay by [CS10].

Remark 1.5. In GP91, the authors claimed that $\overline{\mathbf{B}}_{n}$ is the moduli space for Mustafin joins for arcs, i.e. there exists a family $\overline{\mathbf{F}}_{n} \rightarrow \overline{\mathbf{B}}_{n}$ with the following universal property. Let $\mathbf{a}: \operatorname{Spec}(K) \rightarrow \mathbf{B}_{n}$ be an arc and let $\overline{\mathbf{a}}: \operatorname{Spec}(R) \rightarrow \overline{\mathbf{B}}_{n}$ be its unique extension. Then the pullback of $\overline{\mathbf{F}}_{n}$ via $\overline{\mathbf{a}}$ is isomorphic to $\mathbb{P}\left(\Sigma_{\mathbf{a}}\right)$. However, we found a mistake in the argument and the statement is also wrong, as there exist $\operatorname{arcs} \mathbf{a}, \mathbf{b}: \operatorname{Spec}(K) \rightarrow \mathbf{B}_{n}$ with $\overline{\mathbf{a}}(0)=\overline{\mathbf{b}}(0) \in$ $\overline{\mathbf{B}}_{n}$ such that the central fibers of $\mathbb{P}\left(\Sigma_{\mathbf{a}}\right)$ and $\mathbb{P}\left(\Sigma_{\mathbf{b}}\right)$ are not isomorphic (see Example 7.1).

In \$7, we construct the correct modular compactification of $\mathbf{B}_{n}$ and the universal Mustafin join of point configurations over it using multigraded Hilbert schemes [HS04].

Theorem 1.6. There exists a compactification $\mathbf{B}_{n} \subseteq \overline{\mathbf{X}}_{\mathrm{GP}}(3, n)$ with a proper flat family $\overline{\mathcal{M}} \rightarrow \overline{\mathbf{X}}_{\mathrm{GP}}(3, n)$ smooth along $n$ disjoint sections satisfying the following universal property. If $\mathbf{a}: \operatorname{Spec}(K) \rightarrow \mathbf{B}_{n}$ and $\overline{\mathbf{a}}: \operatorname{Spec}(R) \rightarrow \overline{\mathbf{X}}_{\mathrm{GP}}(3, n)$ is the unique extension, then $\overline{\mathbf{a}}^{*} \overline{\mathcal{M}}$ is isomorphic to the Mustafin join $\mathbb{P}\left(\Sigma_{\mathbf{a}}\right)$ with $n$ sections.

In Proposition 7.10, we construct forgetful morphisms $\overline{\mathbf{X}}_{\mathrm{GP}}(3, n) \rightarrow \overline{\mathbf{X}}_{\mathrm{GP}}(3, n-1)$, the analogues of forgetful morphisms $\overline{\mathrm{M}}_{0, n} \rightarrow \overline{\mathrm{M}}_{0, n-1}$. We further show that there exists a birational morphism $\overline{\mathbf{X}}_{\mathrm{GP}}(3, n) \rightarrow \overline{\mathbf{B}}_{n}$. In Theorem 7.9 , we show that

$$
\overline{\mathrm{X}}_{\mathrm{GP}}(3,5) \cong \overline{\mathrm{X}}(3,5) \cong \overline{\mathbf{B}}_{5} \cong \overline{\mathrm{M}}_{0,5}
$$

However, $\overline{\mathbf{X}}_{\mathrm{GP}}(3,6)$ is different from Kapranov's Chow quotient compactification $\overline{\mathbf{X}}(3,6)$. In Lux08, Luxton showed that $\overline{\mathbf{X}}(3,6)$ is a tropical compactification of $\mathbf{X}(3,6)$ inside the toric variety whose fan $\Sigma(3,6)$ is the tropical Grassmannian of Speyer and Sturmfels [SS04]. The 15 singular points of $\overline{\mathbf{X}}(3,6)$ correspond to non simplicial bipyramid cones in $\Sigma(3,6)$.

Theorem 1.7. $\overline{\mathbf{X}}_{\mathrm{GP}}(3,6)^{\nu}$ is the blow up of $\overline{\mathbf{X}}(3,6)$ given by the minimal refinement of $\Sigma(3,6)$ induced by splitting each bipyramid cone into 12 subcones as illustrated in Table 10. The 6-pointed degenerations of $\mathbb{P}^{2}$ parametrized by $\overline{\mathbf{X}}_{\mathrm{GP}}(3,6)^{\nu}$ are listed in Tables $13-16$.

The main difficulty in dealing with $\overline{\mathbf{X}}(3, n)$ is that its singularities are hard to control. In Theorem 11.2, we show that $\overline{\mathbf{X}}(3, n)^{\nu}$ has a natural open locus $\mathbf{U}(3, n)$ with toroidal singularities. Moreover, its preimage $\mathbf{U}_{\mathrm{GP}}(3, n)$ in $\overline{\mathbf{X}}_{\mathrm{GP}}(3, n)^{\nu}$ is also toroidal. We call 
$\mathbf{U}(3, n)$ and $\mathbf{U}_{\mathrm{GP}}(3, n)$ planar loci because they correspond to limits of arcs with all stable lattices in one apartment of the Bruhat-Tits building. Planar loci are covered by charts which are isomorphic to (non-toric) open subsets in toric varieties with fans that we denote by $\mathcal{Q}_{m}$ and $\widetilde{\mathcal{Q}}_{m}$ (here $n=m+3$ ). While $\mathcal{Q}_{m}$ is nothing but the quotient fan of KapranovSturmfels-Zelevinsky [KSZ91], see $\$ 8$, the fan $\widetilde{\mathcal{Q}}_{m}$ is new, see $\$ 9$.

For $n=6, \mathbf{U}(3,6)$ nearly describes all of $\overline{\mathbf{X}}(3,6)$ (including its singular locus), which allows us to immediately describe $\mathbf{U}_{\mathrm{GP}}(3,6)$ by toric geometry. This leaves a few closed subsets, which we deal with an ad hoc analysis.

Acknowledgements. We would like to thank Valery Alexeev, Dustin Cartwright, Patricio Gallardo, and Sean Keel for helpful conversations related to this work. In particular, we would like to thank Valery Alexeev for allowing us to reproduce in the current paper his pictures [Ale15, Figures 5.11, 5.12, and 5.13]. Finally, we thank the anonymous referee for the valuable comments and feedback. At KTH, the first author was supported by the KTH grant Verg Foundation. The second author was supported by the NSF grant DMS1701704, Simons Fellowship, and by the HSE University Basic Research Program and Russian Academic Excellence Project 5-100.

\section{Contents}

1. Introduction 1

2. A result in matroid theory 4

3. Cross-ratios on Lafforgue's varieties 8

4. Gerritzen and Piwek's cross-ratio variety $\overline{\mathbf{B}}_{n}$

5. Mustafin joins: general theory 13

6. Stable lattices of arcs and their Mustafin joins 17

7. Universal Mustafin join for arcs 21

8. Analogue of the Losev-Manin space for moduli of points in $\mathbb{P}^{2} \quad 30$

9. Toric analogue of $\overline{\mathbf{X}}_{\mathrm{GP}}(3, n) \quad 35$

10. Projective duality and the quotient fans $\mathcal{Q}_{m}$ and $\mathcal{Q}_{m}^{\vee}$

11. Planar locus in $\overline{\mathbf{X}}(3, n)$ and $\overline{\mathbf{X}}_{\mathrm{GP}}(3, n) \quad 46$

12. Study of $\overline{\mathbf{X}}_{\mathrm{GP}}(3,6) \quad 49$

References

\section{A Result in MATROID THEORY}

This section is elementary and we only use standard terminology and facts in matroid theory that can be found in Oxl11]. We use the notation $[n]=\{1, \ldots, n\}$ throughout the paper. Recall that an example of a matroid on $[n]$ is a configuration of $n$ hyperplanes in $\mathbb{P}^{r-1}$. Matroids of this form are called realizable.

Definition 2.1. Let $M$ be a matroid on $[n]$ and let $i_{0} \in[n]$. 
(1) The contraction $M / i_{0}$ is the matroid on $[n] \backslash\left\{i_{0}\right\}$ such that $I \subseteq[n] \backslash\left\{i_{0}\right\}$ is independent provided $I \cup\left\{i_{0}\right\}$ is independent for $M$;

(2) The deletion $M \backslash i_{0}$ is the matroid on $[n] \backslash\left\{i_{0}\right\}$ such that $I \subseteq[n] \backslash\left\{i_{0}\right\}$ is independent provided $I$ is independent for $M$.

(3) $M$ is called disconnected if there exists a partition $[n]=E_{1} \amalg E_{2}$ and matroids $M_{1}, M_{2}$ on $E_{1}, E_{2}$ respectively such that $I$ is an independent set for $M$ if and only if $I=I_{1} \amalg I_{2}$, where $I_{i}$ is an independent set for $M_{i}, i=1,2$. A matroid is connected provided it is not disconnected.

For realizable matroids, deletion corresponds to removing a hyperplane from the arrangement and contraction corresponds to intersecting the arrangement with one of its hyperplanes.

Definition 2.2 ([Tev07]). Let $M$ and $M^{\prime}$ be two matroids on $[n]$. Then we say that $M$ is more constrained than $M^{\prime}$ provided every independent set for $M$ is independent for $M^{\prime}$, but there exists an independent set for $M^{\prime}$ that is dependent for $M$.

The goal of this section is to prove the following:

Theorem 2.3. Let $M$ and $M^{\prime}$ be two matroids on $[n]$ of the same rank $r$. Suppose that $r \geq 3$ or $r=2$ and $n \geq 5$. Then the following statement $(C D)$ holds:

(CD) If $M$ is connected and more constrained than $M^{\prime}$ then there exists $i_{0} \in[n]$ such that either the contraction $M / i_{0}$ is connected and more constrained than $M^{\prime} / i_{0}$, or the deletion $M \backslash i_{0}$ is connected and more constrained than $M^{\prime} \backslash i_{0}$.

Before we plunge into the proof, we recast the theorem in the language of matroid polytopes. Let $\left\{e_{1}, \ldots, e_{n}\right\}$ be the basis of $\mathbb{R}^{n}$. The hypersimplex $\Delta(r, n)$ is the lattice polytope

$$
\Delta(r, n)=\text { ConvHull }\left(\sum_{i \in I} e_{i} \mid I \subseteq[n] \text { and }|I|=r\right) \subseteq \mathbb{R}^{n} .
$$

A matroid polytope $P$ in $\mathbb{R}^{n}$ is a lattice polytope whose vertices are $\sum_{i \in B} e_{i}$, where $B$ varies among the bases of a matroid on $[n]$. If the matroid has rank $r$, then the vertices of $P$ are also vertices of the hypersimplex $\Delta(r, n)$. The hypersimplex is the matroid polytope of the uniform matroid. Recall that $M$ is connected if and only if $P$ has dimension $n-1$, see [Laf03, Theorem 1.11] or [FS05, Proposition 2.4]. If a matroid $M$ is more constrained than a matroid $M^{\prime}$, then we have $P \subsetneq P^{\prime}$ for the corresponding matroid polytopes. The converse holds if we assume $M$ and $M^{\prime}$ have the same rank. So we have

Corollary 2.4. Let $P, P^{\prime} \subseteq \Delta(r, n)$ be matroid polytopes of rank $r$ and dimension $n-1$ such that $r \geq 3$, or $r=2$ and $n \geq 5$. Assume $P \subsetneq P^{\prime}$. Then there exists a facet $F \subseteq \Delta(r, n)$ such that $\left.P\right|_{F}$ and $\left.P^{\prime}\right|_{F}$ are facets of $P$ and $P^{\prime}$ respectively, and $\left.\left.P\right|_{F} \subsetneq P^{\prime}\right|_{F}$.

Proof. Let $M, M^{\prime}$ be the matroids corresponding to $P, P^{\prime}$. By Theorem 2.3 , there exists $i_{0} \in[n]$ such that either $M / i_{0}$ is connected and more constrained than $M^{\prime} / i_{0}$, or $M \backslash i_{0}$ is connected and more constrained than $M^{\prime} \backslash i_{0}$. In the former case, let $F$ be the facet of $\Delta(r, n)$ given by the hyperplane $x_{i_{0}}=1$. Then $\left.P\right|_{F}$ and $\left.P^{\prime}\right|_{F}$ are the matroid polytopes corresponding to $M / i_{0}$ and $M^{\prime} / i_{0}$ respectively. Therefore, $\left.\left.P\right|_{F} \subsetneq P^{\prime}\right|_{F}$. In the latter case we consider the facet of $\Delta(r, n)$ given by the hyperplane $x_{i_{0}}=0$. 
Next we consider matroid polytope subdivisions of a matroid polytope.

Corollary 2.5. Let $Q \subseteq \Delta(r, n)$ be a matroid polytope of rank $r$ and dimension $n-1$ such that $r \geq 3$, or $r=2$ and $n \geq 5$. Let $\mathscr{P}, \mathscr{P}^{\prime}$ be two subdivisions of $Q$ into matroid polytopes such that $\mathscr{P}^{\prime}$ is coarser than $\mathscr{P}$. Then there exists a facet $F \subseteq \Delta(r, n)$ such that $E=\left.Q\right|_{F}$ is a facet of $Q$ and $\left.\mathscr{P}^{\prime}\right|_{E}$ is coarser than $\left.\mathscr{P}\right|_{E}$.

Proof. Since $\mathscr{P}^{\prime}$ is coarser than $\mathscr{P}$, we can find maximal dimensional polytopes $P \in \mathscr{P}$ and $P^{\prime} \in \mathscr{P}^{\prime}$ such that $P \subsetneq P^{\prime}$. By Corollary 2.4 there exists a facet $F \subseteq \Delta(r, n)$ such that $\left.P\right|_{F}$ and $\left.P^{\prime}\right|_{F}$ are facets of $P$ and $P^{\prime}$ respectively, and $\left.\left.P\right|_{F} \subsetneq P^{\prime}\right|_{F}$. If we set $E=\left.Q\right|_{F}$, it follows that the subdivision $\left.\mathscr{P}^{\prime}\right|_{E}$ is coarser than $\left.\mathscr{P}\right|_{E}$.

The rest of this section is dedicated to the proof of Theorem 2.3 .

Lemma 2.6. Let $M$ and $M^{\prime}$ be two matroids on $[n]$. Assume that $M$ is more constrained than $M^{\prime}$ and let $I^{\prime}$ be an independent set for $M^{\prime}$ which is dependent for $M$. Then

(a) If $i_{0} \in I^{\prime}$, then $M / i_{0}$ is more constrained than $M^{\prime} / i_{0}$;

(b) If $j_{0} \in[n] \backslash I^{\prime}$, then $M \backslash j_{0}$ is more constrained than $M^{\prime} \backslash j_{0}$.

Proof. If $I$ is independent for $M / i_{0}$, then $I \cup\left\{i_{0}\right\}$ is independent for $M$ by definition. Hence, $I \cup\left\{i_{0}\right\}$ is independent for $M^{\prime}$, implying that $I$ is independent for $M^{\prime} / i_{0}$. Moreover, $I^{\prime} \backslash\left\{i_{0}\right\}$ is independent for $M^{\prime} / i_{0}$, but dependent for $M / i_{0}$. If $I$ is independent for $M \backslash j_{0}$, then $I$ is independent for $M$. Hence, $I$ is independent for $M^{\prime}$, implying that $I$ is independent for $M^{\prime} \backslash j_{0}$ because $j_{0} \notin I$. Moreover, $I^{\prime}$ is independent for $M^{\prime} \backslash j_{0}$, but dependent for $M \backslash j_{0}$.

Proposition 2.7. Let $M$ and $M^{\prime}$ be two matroids on $[n]$ of the same rank. Assume that at least two distinct independent sets $I_{1}^{\prime}, I_{2}^{\prime}$ for $M^{\prime}$ are dependent for $M$. Then $(C D)$ holds.

Proof. Up to switching $I_{1}^{\prime}$ with $I_{2}^{\prime}$, let $e \in I_{1}^{\prime} \backslash I_{2}^{\prime}$. If $M / e$ is connected, then we are done by Lemma 2.6 (a). Otherwise, by [Oxl11, Theorem 4.3.1] we have that $M \backslash e$ is connected, and by Lemma 2.6 (b) we have that $M \backslash e$ is more constrained than $M^{\prime} \backslash e$.

Proposition 2.8. Let $M$ and $M^{\prime}$ be two matroids on $[n]$ of the same rank. Assume that $M$ has no size two cocircuit. Then (CD) holds.

Proof. By Proposition 2.7 we can assume there is a unique independent set $I^{\prime}$ for $M^{\prime}$ that is dependent for $M$. Observe that $I^{\prime}$ must be a basis of $M^{\prime}$, hence $\left|I^{\prime}\right|=r$, where $r$ is the common rank of $M$ and $M^{\prime}$. From the connectedness of $M$ we have that $M$ has no coloops: $M$ connected implies that the dual matroid $M^{*}$ is also connected by [Oxl11, Corollary 4.2.5], and a connected matroid has no loops, so $M^{*}$ has no loops, implying that $M$ has no coloops. Moreover, by hypothesis $M$ has no size two cocircuits, hence $M$ is a cosimple matroid (by definition, a matroid is cosimple provided it has no coloops and no size two cocircuits). This implies that the dual matroid $M^{*}$ is simple and connected. Hence there are at least $r+1$ elements $e$ such that $M^{*} / e$ is connected by [Oxl11, $\S 4.3$, Exercise 10 (e)]. In particular, we can find one of such $e$ in the complement of $I^{\prime}$. Since $M^{*} / e$ is connected, also $\left(M^{*} / e\right)^{*}=M \backslash e$ is connected (here we used [Oxl11, §3.1, Exercise $1(\mathrm{~b})]$ and $\left(M^{*}\right)^{*}=M$ ), and by Lemma 2.6 (b) we have that $M \backslash e$ is more constrained than $M^{\prime} \backslash e$. 
Lemma 2.9. Let $M$ and $M^{\prime}$ be two matroids on $[n]$ of the same rank. Assume that $M$ is more constrained than $M^{\prime}$. Then the dual matroid $M^{*}$ is more constrained than $\left(M^{\prime}\right)^{*}$.

Proof. Let $I$ be an independent set for $M^{*}$. Then there exists a basis $B$ for $M$ disjoint from $I$. But $B$ is also a basis for $M^{\prime}$ because $M$ is more constrained than $M^{\prime}$ and they have the same rank. So $I$ is also independent for $M^{\prime}$. Let $I^{\prime}$ be an independent set for $M^{\prime}$ that is dependent for $M$. Let $B^{\prime}$ be a basis for $M^{\prime}$ containing $I^{\prime}$. Then $B^{\prime}$ is also dependent for $M$. It follows that $\left(B^{\prime}\right)^{c}$ is a basis for $\left(M^{\prime}\right)^{*}$ and $\left(B^{\prime}\right)^{c}$ is dependent for $M^{*}$, proving that $M^{*}$ is more constrained than $\left(M^{\prime}\right)^{*}$.

Proof of Theorem 2.3. Suppose first that $r \geq 3$. By Lemma 2.9, we have that the dual matroid $M^{*}$ is connected and more constrained than $\left(M^{\prime}\right)^{*}$. If there exists $i_{0} \in[n]$ such that $M^{*} / i_{0}$ is connected and more constrained than $\left(M^{\prime}\right)^{*} / i_{0}$, or $M^{*} \backslash i_{0}$ is connected and more constrained than $\left(M^{\prime}\right)^{*} \backslash i_{0}$, then $M \backslash i_{0}$ is connected and more constrained than $M^{\prime} \backslash i_{0}$, or $M / i_{0}$ is connected and more constrained than $M^{\prime} / i_{0}$ respectively. Therefore, by Proposition 2.8 applied to $M^{*}$ and $\left(M^{\prime}\right)^{*}$, we can assume that $M^{*}$ has a size two cocircuit $C$, which is a size two circuit of $M$.

By Proposition 2.7 we can assume that there exists a unique independent set for $M^{\prime}$ that is dependent for $M$. Recall that $I^{\prime}$ must be a basis of $M^{\prime}$, hence $\left|I^{\prime}\right|=r$ and in particular $I^{\prime} \neq C$ (here is where we used the assumption that $r \geq 3$ ). Therefore, $C$ must be dependent for $M^{\prime}$, otherwise we would contradict the uniqueness of $I^{\prime}$. In particular, $C \nsubseteq I^{\prime}$, hence we can find an element $c \in C \backslash I^{\prime}$. By Lemma 2.6 (b), we only need to show that $M \backslash c$ is connected. But this follows from Lemma 2.10 below.

Finally, suppose $r=2$ and $n \geq 5$. First of all observe that $M$ (and similarly $M^{\prime}$ ) is realized by a point arrangement in $\mathbb{P}^{1}$ (over the complex numbers). Let $\mathcal{H}=\left\{p_{1}, \ldots, p_{n}\right\}$, and declare $p_{i}=p_{j}$ for $i \neq i$ provided $\{i, j\}$ is a circuit of $M$. Then the matroid associated to $\mathcal{H}$ is isomorphic to $M$. Similarly, define $\mathcal{H}^{\prime}=\left\{p_{1}^{\prime}, \ldots, p_{n}^{\prime}\right\}$.

For point configurations on $\mathbb{P}^{1}$, saying that $\mathcal{H}$ is more constrained than $\mathcal{H}^{\prime}$ means that if some points coincide in $\mathcal{H}^{\prime}$, then the corresponding points in $\mathcal{H}$ coincide as well, but not vice versa. In what follows we analyze an exhaustive list of possibilities where in each one we determine an appropriate index $i_{0}$.

If there exist $i, j \in[n]$ with $i \neq j$ such that $p_{i}^{\prime}=p_{j}^{\prime}$, then one can take $i_{0}=i$ and the claim follows. So let us assume all points in $\mathcal{H}^{\prime}$ are distinct. Under this assumption, what we need to show is that we can always find $i_{0} \in[n]$ such that $\mathcal{H} \backslash i_{0}$ is automorphism-free and it contains points appearing multiple times.

If $p_{i} \in \mathcal{H}$ appears at least three times, then we can set $i_{0}=i$. Otherwise, assume each point in $\mathcal{H}$ appears at most two times. If $p_{i} \in \mathcal{H}$ is the only point appearing twice, then let $i_{0} \in[n] \backslash\{i\}$ (in this case the hypothesis $n>4$ guarantees that $\mathcal{H} \backslash i_{0}$ is automorphism-free). If there exist two distinct points $p_{i}, p_{j} \in \mathcal{H}$ appearing twice, then we can set $i_{0}=i$. Since we considered all the possibilities, this concludes the proof.

Lemma 2.10. Let $M$ be a connected matroid and let $C$ be a size two circuit of $M$. Then for all $c \in C$ we have that $M \backslash c$ is connected. 
Proof. Let $C=\left\{c_{1}, c_{2}\right\}$. Let us show $M \backslash c_{1}$ is connected (connectedness of $M \backslash c_{2}$ is proved analogously). We show that for each $x \neq c_{1}, c_{2}$ in the ground set, there exists a circuit of $M \backslash c_{1}$ containing both $x$ and $c_{2}$, so that $x$ and $c_{2}$ belong to the same connected component. Since $M$ is connected, there exists a circuit $D$ of $M$ containing $x$ and $c_{2}$. Observe that $c_{1} \notin D$ because $C \nsubseteq D$ by the minimality of $D$. Hence, $D$ is also a circuit of $M \backslash c_{1}$.

Remark 2.11. The assumption $n \geq 5$ if $r=2$ can not be removed. Let $M^{\prime}=U_{2,4}$ be the uniform matroid of rank 2 with ground set [4]. Let $M=\widetilde{U}_{2,4}$ be the matroid with the same ground set and with bases

$$
\{1,2\},\{1,3\},\{1,4\},\{2,3\},\{2,4\} .
$$

It is easy to check that (CD) does not hold.

\section{Cross-Ratios on LAfForgue's varieties}

We recall several definitions and facts from Kap93a, Laf03, KT06]. Let $G(r, n)$ denote the Grassmannian of $r$-dimensional linear subspaces in $\mathbb{k}^{n}$ embedded in

$$
\mathbb{P}:=\mathbb{P}\left(\bigwedge^{r} \mathbb{k}^{n}\right)
$$

via the Plücker embedding. Let $G^{0}(r, n) \subseteq G(r, n)$ be the subset of points with nonzero Plücker coordinates. The torus $H=\mathbb{G}_{m}^{n} / \operatorname{diag}\left(\mathbb{G}_{m}\right)$ acts on $G(r, n)$ via the action of $\mathbb{G}_{m}^{n}$ on $\mathbb{k}^{n}$ and $G^{0}(r, n)$ is an $H$-invariant open subset. Denote the quotient by $\mathbf{X}(r, n)=G^{0}(r, n) / H$ ( $H$ acts freely). By the Gelfand-MacPherson correspondence [GM82, $\mathbf{X}(r, n)$ is also the moduli space of $n$ hyperplanes in general linear position in $\mathbb{P}^{r-1}$. Namely, a point of $G(r, n)$ can be represented as the row space of an $r \times n$ matrix, and columns of this matrix give $n$ hyperplanes in $\mathbb{P}^{r-1}$. Kapranov's compactification $\overline{\mathbf{X}}(r, n)$ of $\mathbf{X}(r, n)$ is the Chow quotient

$$
\overline{\mathbf{X}}(r, n)=G(r, n) / / H .
$$

By $\operatorname{Kap93a}, \overline{\mathbf{X}}(r, n)$ is also isomorphic to the Chow quotient of $\left(\mathbb{P}^{r-1}\right)^{n}$ by $\mathrm{PGL}_{r}$. For example, $\overline{\mathbf{X}}(2, n)$ is isomorphic to $\overline{\mathrm{M}}_{0, n}$, the moduli space of stable genus zero $n$-pointed curves. In particular, $\overline{\mathbf{X}}(2,4)=\mathbb{P}^{1}$ via the cross-ratio of four points.

For $x \in \mathbb{P}$, let $\operatorname{Supp}(x)$ be the convex hull of the vertices of $\Delta(r, n)$ corresponding to nonzero coordinates of $x$. Given a matroid polytope $P \subseteq \Delta(r, n)$, we define the algebraic tori

$$
\mathbb{P}^{P, 0}=\{x \in \mathbb{P} \mid \operatorname{Supp}(x)=P\} \quad \text { and } \quad \mathcal{A}_{0}^{P}:=\mathbb{P}^{P, 0} / H .
$$

For instance, $\mathbb{P}^{\Delta(r, n), 0} \subseteq \mathbb{P}$ is the maximal torus. We refer to GKZ08 for the theory of regular subdivisions and secondary polytopes. We define $\mathcal{F}_{\text {sec }}(P)$ to be the normal fan to the secondary polytope of $P$. The union of cones in $\mathcal{F}_{\text {sec }}(P)$ corresponding to regular subdivisions of $P$ by matroid polytopes is a subfan denoted by $\mathcal{F}_{\text {Laf }}(P)$. The Lafforgue toric variety $\mathcal{A}^{P}$ is the toric variety of $\mathcal{A}_{0}^{P}$ associated to the fan $\mathcal{F}_{\text {Laf }}(P)$. We define $\Omega^{P}$ to be the quotient

$$
\Omega^{P}=\left(G(r, n) \cap \mathbb{P}^{P, 0}\right) / H \subseteq \mathcal{A}_{0}^{P} .
$$


The Lafforgue variety $\bar{\Omega}^{P}$ is a certain projective subscheme of the toric variety $\mathcal{A}^{P}$, the precise definition of which we will not need. $\bar{\Omega}^{P}$ contains $\Omega^{P}$ as an open subset, but in general $\bar{\Omega}^{P}$ is reducible [KT06, Proposition 3.10]. The closure of $\Omega^{P}$ in $\bar{\Omega}^{P}$ is called the main stratum. If $P=\Delta(r, n)$, then $G(r, n) \cap \mathbb{P}^{P, 0}=G^{0}(r, n)$. Therefore, $\boldsymbol{\Omega}^{P}=\mathbf{X}(r, n)$. By [KT06, 2.9], $\overline{\mathbf{X}}(r, n)$ and the main stratum in $\overline{\mathbf{\Omega}}^{P}$ have the same normalization $\overline{\mathbf{X}}(r, n)^{\nu}$.

Remark 3.1. We note that $P \subseteq \Delta(r, n)$ is the matroid polytope of a non-realizable matroid if and only if $\Omega^{P}=\emptyset$ (see [KT06, 2.6]). The main stratum is then of course also empty but we imagine that $\bar{\Omega}^{P}$ can still be non-empty, although we do not know an example.

Definition 3.2. Given a matroid polytope $P$ and a face $F \subseteq P$, we have an induced morphism of Lafforgue's toric varieties $\mathcal{A}^{P} \rightarrow \mathcal{A}^{F}$ by restricting piece-wise affine functions. It is called the face map and restricts to $\bar{\Omega}^{Q}$ giving a morphism

$$
\bar{\Omega}^{Q} \rightarrow \bar{\Omega}^{F}
$$

which we also call a face map. Note that $\Delta(r, n)$ has $2 n$ facets given by hyperplanes $x_{i}=0$ and $x_{i}=1$. These facets are equivalent to $\Delta(r, n-1)$ and $\Delta(r-1, n-1)$, respectively.

Lemma 3.3. Let $Q \subseteq \Delta(r, n)$ be a matroid polytope of rank $r$ and dimension $n-1$ such that either $r \geq 3$ or $r=2$ and $n \geq 5$. Let $\mathcal{F}$ be the collection of facets of $Q$ that are contained in the boundary of $\Delta(r, n)$. Then the fibers of the product of face maps

$$
f: \mathcal{A}^{Q} \rightarrow \prod_{F \in \mathcal{F}} \mathcal{A}^{F}
$$

do not contain any complete subvarieties.

Proof. Let $C \subseteq \mathcal{A}^{Q}$ be a complete curve. Let $S^{\prime} \subseteq \mathcal{A}^{Q}$ be the minimal closed toric stratum containing $C$. More explicitly, we can write $S^{\prime}=\overline{T^{\prime}}$ where $\overline{T^{\prime}} \subseteq \mathcal{A}^{Q}$ is the closure of a torus $T^{\prime}$ of the appropriate dimension. Since the torus $T^{\prime}$ is affine, $C$ is projective, and $C \cap T^{\prime} \neq \emptyset$ by the minimality of $S^{\prime}$, we cannot have that $C \subseteq T^{\prime}$, hence $C$ intersects the closure of another torus orbit $\bar{T} \subsetneq \overline{T^{\prime}}$. Then $S=\bar{T}$ is a toric stratum properly contained in $S^{\prime}$ which intersects $C$ nontrivially. Let $p \in S \cap C$ and $p^{\prime} \in\left(S^{\prime} \backslash S\right) \cap C$.

The stratum $S^{\prime}$ (resp. $S$ ) corresponds to a matroid polytope subdivision $\mathscr{P}^{\prime}$ (resp. $\mathscr{P}$ ) of $Q$. Since $S \subsetneq S^{\prime}$, the subdivision $\mathscr{P}^{\prime}$ is coarser than $\mathscr{P}$. Then we can apply Corollary 2.5 and find a facet $E \in \mathcal{F}$ such that $\left.\mathscr{P}^{\prime}\right|_{E}$ is coarser than $\left.\mathscr{P}\right|_{E}$. Then $\left.\mathscr{P}^{\prime}\right|_{E}$ and $\left.\mathscr{P}\right|_{E}$ correspond to two toric strata $\Sigma \subsetneq \Sigma^{\prime}$ of $\mathcal{A}^{E}$. Let $f_{E}$ be the face map $\mathcal{A}^{Q} \rightarrow \mathcal{A}^{E}$. Then $f_{E}\left(p^{\prime}\right) \in \Sigma^{\prime} \backslash \Sigma$ and $f_{E}(p) \in \Sigma$, implying that $f(p) \neq f\left(p^{\prime}\right)$. Therefore $C$ is not contracted by the morphism $f$. It follows that $f$ does not contract any complete curve.

Definition 3.4. Let $Q \subseteq \Delta(r, n)$ be a matroid polytope of rank $r$. We say that $Q$ is framed provided it is realized by a hyperplane arrangement $\mathcal{H}$ in $\mathbb{P}^{r-1}$ containing $r+1$ hyperplanes in general linear position. Note that such a matroid is automatically connected.

Proof of Theorem 1.2. We apply Lemma 3.3 to $\mathcal{A}^{Q}$ recursively until we map to a product

$$
f^{\prime}: \mathcal{A}^{Q} \rightarrow \prod_{F \in \mathcal{F}^{\prime}} \mathcal{A}^{F}
$$


where $\mathcal{F}^{\prime}$ consists of the faces of $Q$ of rank 2 that are contained in the boundary of $\Delta(r, n)$. We know that the above morphism does not contract any complete curve.

Let $M$ be the matroid associated to $F \in \mathcal{F}^{\prime}$. There are the following two possibilities:

(1) $M$ is isomorphic to $U_{2,4}$, hence $F$ is equivalent to $\Delta(2,4)$;

(2) $M$ is isomorphic to $\widetilde{U}_{2,4}$ (see Remark 2.11).

Therefore, $\mathcal{F}$ is the subset of $\mathcal{F}^{\prime}$ of facets that are not of type (2). Consider the composition

$$
f: \mathcal{A}^{Q} \stackrel{f^{\prime}}{\rightarrow} \prod_{F \in \mathcal{F}^{\prime}} \mathcal{A}^{F} \stackrel{\pi}{\rightarrow} \prod_{F \in \mathcal{F}} \mathcal{A}^{F} .
$$

Then $f$ does not contract any complete curve either. Assume by contradiction $C \subseteq \mathcal{A}^{Q}$ is a complete curve contracted by $f$. We know $f^{\prime}$ does not contract $C$, so $\pi$ contracts $f^{\prime}(C)$. Since $\pi$ contracts all the factors $\mathcal{A}^{F}$ with $F$ of type (2), then $C$ has to map onto some $\mathcal{A}^{F}$ with $F$ of type (2). But $\mathcal{A}^{F} \cong \mathbb{G}_{m}$, which is a contradiction because $C$ is complete and $\mathcal{A}^{F}$ is affine. Note that $\mathcal{A}^{F} \cong \mathbb{G}_{m}$ because $F$ does not have any proper subdivision into matroid subpolytopes. Restricting $f$ to the projective variety $\bar{\Omega}^{Q}$, we obtain the claimed finite morphism.

Finally, suppose that $Q$ is framed. We claim that the product of face maps $(1)$ is a locally closed embedding and thus its restriction to the main stratum of $\bar{\Omega}^{Q}$ is a finite birational morphism. It suffices to show $\Omega^{Q} \rightarrow \prod_{F \in \mathcal{F}} \bar{\Omega}^{F}$ is injective on $R$-points, where $(R, \mathfrak{m})$ is any local $\mathbb{k}$-algebra with $R / \mathfrak{m} \cong \mathbb{k}$. Let $p \in \Omega^{Q}(R)$ and let $\left(\mathbb{P}_{R}^{r-1}, \sum_{i=1}^{n} H_{i}\right)$ be the hyperplane arrangement parametrized by it (the projective bundle is trivial because the frame gives a section of the associated $\mathrm{PGL}_{r}$-torsor). The images of $p$ under the face maps (in other words, the cross-ratios of the hyperplane arrangement) are described as follows. Let $a_{i}$ be the vector of coefficients of the hyperplane $H_{i}$. Fix distinct indices $i_{1}, \ldots, i_{r-2}, i, j, k, \ell \in[n]$ (these determine a face $F \in \mathcal{F}$ ). Then the corresponding cross-ratio is given by the point

$$
\left[\left|a_{i_{1}}, \ldots, a_{i_{r-2}}, a_{i}, a_{j}\right|\left|a_{i_{1}}, \ldots, a_{i_{r-2}}, a_{k}, a_{\ell}\right|:\left|a_{i_{1}}, \ldots, a_{i_{r-2}}, a_{i}, a_{k}\right|\left|a_{i_{1}}, \ldots, a_{i_{r-2}}, a_{j}, a_{\ell}\right|\right] \in \mathbb{P}^{1} .
$$

We show that the hyperplane arrangement $\left(\mathbb{P}_{R}^{r-1}, \sum_{i=1}^{n} H_{i}\right)$ can be uniquely reconstructed by the data of these cross-ratios. Since $R$ is local, the determinant of the matrix of coefficients of any $r$ hyperplanes among $H_{1}, \ldots, H_{r+1}$ is invertible. In particular, up to $\mathrm{PGL}_{r}(R)$-action, we can assume $H_{1}, \ldots, H_{r+1}$ are the standard hyperplanes. In other words, we can assume $\left(a_{1}, \ldots, a_{r+1}\right)=\left(\operatorname{Id}_{r}, \mathbf{1}\right)$, where $\operatorname{Id}_{r}$ is the $r \times r$ identity matrix and $\mathbf{1}=(1, \ldots, 1)$. We show that any $b \in\left\{a_{r+2}, \ldots, a_{n}\right\}$ is uniquely determined by the cross-ratios. Consider the cross-ratio corresponding to the choice $\left(i_{1}, \ldots, i_{r-2}\right)=(1, \ldots, r-2), i=r+1, a_{j}=b, k=$ $r-1, \ell=r$, which is given by $\left[b_{r-1}-b_{r}: b_{r-1}\right]$. This fixes $\frac{b_{r}}{b_{r-1}} \in \mathbb{P}^{1}$. In general, by letting $\left(i_{1}, \ldots, i_{r-2}\right)=(1, \ldots, \widehat{s}, \ldots, \widehat{t}, \ldots, r), i=r+1, a_{j}=b, k=s, \ell=s+1$, we can fix the ratio $\frac{b_{t}}{b_{s}}$, hence also $\frac{b_{s}}{b_{t}}$. Therefore, to determine $b$, let $b_{s}$ be one of the nonzero coordinates of $b$ (there exists at least one because $b$ represents the coefficients of a hyperplane). Then all the ratios $\frac{b_{1}}{b_{s}}, \ldots, \frac{b_{r}}{b_{s}}$ are fixed, hence the coefficients $b_{1}, \ldots, b_{r}$ are uniquely determined up to a common scalar, proving what we wanted. 
Corollary 3.5. Let $r \geq 2$ and $n \geq r+2$. The product of forgetful morphisms

$$
\overline{\mathbf{X}}(r, n) \rightarrow \prod_{I \in\left(\begin{array}{c}
{[n]} \\
r+2
\end{array}\right)} \overline{\mathbf{X}}(r, I)
$$

and the rational map $\overline{\mathbf{X}}(r, n) \rightarrow \prod_{v_{1}, \ldots, v_{r+2} \in\{1, \ldots, n\}} \mathbb{P}^{1}$ are finite birational morphisms onto their image. For $r \geq 3$, each map $\overline{\mathbf{X}}(r, n) \rightarrow \mathbb{P}^{1}$ is the cross-ratio of 4 points obtained by intersecting hyperplanes $H_{v_{1}}, H_{v_{2}}, H_{v_{3}}, H_{v_{4}}$ with the line $H_{v_{5}} \cap \ldots \cap H_{v_{r+2}}$.

Proof. Consider the product $\prod_{F \in \mathcal{F}} \bar{\Omega}^{F}$ in Theorem 1.2 for $Q=\Delta(r, n)$. Each $\bar{\Omega}^{F} \cong \overline{\mathrm{M}}_{0,4}$ and the face $F$ can be described as follows. Choose distinct indices $i, j, k, \ell, i_{1}, \ldots, i_{r-2} \in[n]$. Then $F$ is the convex hull of the set $\left\{e_{a}+e_{b}+e_{i_{1}}+\ldots+e_{i_{r-2}} \mid a, b \in\{i, j, k, \ell\}\right\}$. This gives the following identification:

$$
\prod_{I \in\left(\begin{array}{c}
n n] \\
r+2
\end{array}\right)} \prod_{J \in\left(\begin{array}{c}
I \\
r-2
\end{array}\right)} \overline{\mathrm{M}}_{0, I \backslash J} \cong \prod_{F \in \mathcal{F}} \bar{\Omega}^{F} .
$$

For each $I \in\left(\begin{array}{c}{[n]} \\ r+2\end{array}\right)$, we have isomorphisms $\overline{\mathbf{X}}(r, I) \cong \overline{\mathbf{X}}(2, I) \cong \overline{\mathrm{M}}_{0, I}$. We claim that $f: \overline{\mathbf{X}}(r, n) \rightarrow \prod_{I \in\left(\begin{array}{l}{[n]} \\ r+2\end{array}\right)} \overline{\mathrm{M}}_{0, I}$ is finite and birational onto its image. Given $I \in\left(\begin{array}{c}{[n]} \\ r+2\end{array}\right)$, the product of forgetful morphisms $\overline{\mathrm{M}}_{0, I} \rightarrow \prod_{J \in\left(\begin{array}{c}I \\ r-2\end{array}\right)} \overline{\mathrm{M}}_{0, I \backslash J}$ is a closed embedding [HKT09, Theorem 9.18]. Now consider the following commutative diagram:

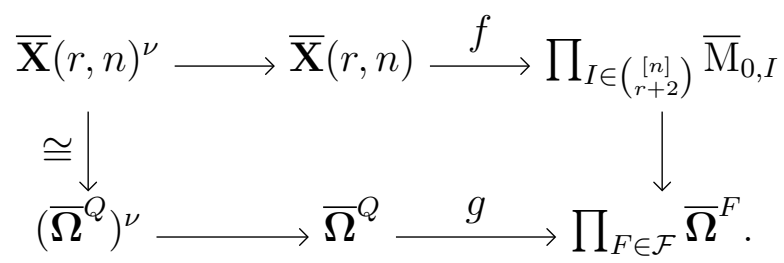

Since $g$ is finite and birational onto its image by Theorem 1.2 , the morphism $f$ also is.

\section{Gerritzen and PiWeK's Cross-Ratio VAriety $\overline{\mathbf{B}}_{n}$}

We start by recalling the definition of the Gerritzen-Piwek's compactification $\overline{\mathbf{B}}_{n}$ GP91]. Let $\mathbf{U}_{n} \subseteq\left(\mathbb{P}^{2}\right)^{n}$ be an open subset parametrizing configurations $\left(x_{1}, \ldots, x_{n}\right)$ of $n$ distinct points in $\mathbb{P}^{2}$ in general linear position and let $\mathbf{B}_{n}$ be the moduli space, the quotient of $\mathbf{U}_{n}$ by the free action of $\mathrm{PGL}_{3}$. We have the quotient morphism $\mathbf{U}_{n} \rightarrow \mathbf{B}_{n}$. If we normalize the points $x_{1}, x_{2}, x_{3}, x_{4}$ to be $e_{1}=[1: 0: 0], e_{2}=[0: 1: 0], e_{3}=[0: 0: 1], e_{4}=[1: 1: 1]$ applying a unique projective transformation, we obtain a section

$$
s: \mathbf{B}_{n} \rightarrow \mathbf{U}_{n}
$$

To "symmetrize" this map, let $\mathcal{Q}_{n}$ be the set of ordered 5-tuples of points and let $v \in \mathcal{Q}_{n}$. Define the map $q_{v}: \mathbf{B}_{n} \rightarrow \mathbb{P}^{2}$ as the image of the point $x_{v_{5}}$ under a unique projective 
transformation $\psi_{x_{v_{1}}, \ldots, x_{v_{4}}} \in \mathrm{PGL}_{3}$ that sends $x_{v_{1}}, x_{v_{2}}, x_{v_{3}}, x_{v_{4}}$ to $e_{1}, e_{2}, e_{3}, e_{4}$, respectively. The product morphism

$$
\prod_{v \in \mathcal{Q}_{n}} q_{v}: \mathbf{B}_{n} \rightarrow \prod_{\mathcal{Q}_{n}} \mathbb{P}^{2}
$$

is an open immersion onto a closed subscheme of $\prod_{\mathcal{Q}_{n}} \mathbb{P}^{2}$. The Gerritzen-Piwek compactification $\overline{\mathbf{B}}_{n}$ is the closure of $\mathbf{B}_{n}$ in $\prod_{\mathcal{Q}_{n}} \mathbb{P}^{2}$ under the above immersion. (Note that in GP91, the compact moduli space is denoted by $\mathbf{B}_{n}$ and its interior is denoted by $\mathbf{B}_{n}^{*}$.) A slightly more economical embedding can be obtained as follows.

Lemma 4.1. Let $\mathcal{Q}_{n}^{\prime}$ denote the set of ordered 5-tuples in $[n]$ where the first four elements are in increasing order. $\overline{\mathbf{B}}_{n}$ is isomorphic to the closure of the image of $\mathbf{B}_{n}$ in $\prod_{\mathcal{Q}_{n}^{\prime}} \mathbb{P}^{2}$ under the product of forgetful maps.

Proof. There is a map $\mu: \mathcal{Q}_{n} \rightarrow \mathcal{Q}_{n}^{\prime}$ sending $v=\left(v_{1}, \ldots, v_{5}\right)$ to $\left(v_{\tau(v)(1)}, \ldots, v_{\tau(v)(4)}, v_{5}\right)$, where $\tau: \mathcal{Q}_{n} \rightarrow S_{4}$ is the map that associates to $v \in \mathcal{Q}_{n}$ the unique permutation $\tau(v) \in S_{4}$ such that $v_{\tau(v)(1)}<\ldots<v_{\tau(v)(4)}$. Given $\sigma \in S_{4}$, let $\varphi_{\sigma}$ be the unique projective linear transformation of $\mathbb{P}^{2}$ such that $\varphi_{\sigma}\left(e_{i}\right)=e_{\sigma(i)}$ for all $i=1, \ldots, 4$. Let $f: \prod_{\mathcal{Q}_{n}^{\prime}} \mathbb{P}^{2} \rightarrow \prod_{\mathcal{Q}_{n}} \mathbb{P}^{2}$ be the map $\left(p_{v}\right)_{v \in \mathcal{Q}_{n}^{\prime}} \mapsto\left(\varphi_{\tau(v)}\left(p_{\mu(v)}\right)\right)_{v \in \mathcal{Q}_{n}}$. Let $\pi: \prod_{\mathcal{Q}_{n}} \mathbb{P}^{2} \rightarrow \prod_{\mathcal{Q}_{n}^{\prime}} \mathbb{P}^{2}$ be the natural product of projection maps. Then $f$ is a section of $\pi$ and we have a commutative diagram

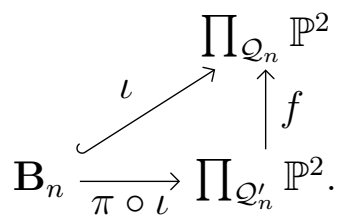

The result follows.

Next we prove Theorem 1.1 there exists a finite birational morphism $\overline{\mathbf{X}}(3, n) \rightarrow \overline{\mathbf{B}}_{n}$. Proof of Theorem 1.1. Consider the following commutative diagram:

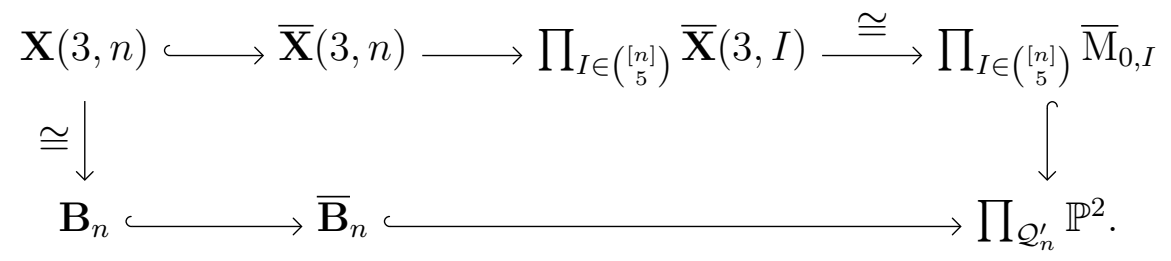

Recall that the morphism $\overline{\mathbf{X}}(3, n) \rightarrow \prod_{I \in\left(\begin{array}{c}{[n]} \\ 5\end{array}\right)} \overline{\mathbf{X}}(3, I)$ is finite and birational onto its image by Corollary 3.5. The embedding $\prod_{I \in\left(\begin{array}{c}{[n]} \\ 5\end{array}\right)} \overline{\mathrm{M}}_{0, I} \hookrightarrow \prod_{\mathcal{Q}_{n}^{\prime}} \mathbb{P}^{2}$ is obtained by applying Lemma 4.2 to each copy of $\overline{\mathrm{M}}_{0, I}$. This gives a finite birational morphism $\overline{\mathrm{X}}(3, n) \rightarrow \overline{\mathbf{B}}_{n}$.

For the next lemma, recall that the $i$-th Kapranov's map [Kap93b is a birational morphism $\overline{\mathrm{M}}_{0, n} \rightarrow \mathbb{P}^{n-3}$ given by the linear system $\left|\psi_{i}\right|$. 
Lemma 4.2. The product of Kapranov's maps $\overline{\mathrm{M}}_{0,5} \rightarrow \prod_{i=1}^{5} \mathbb{P}^{2}$ is a closed embedding.

Proof. The boundary of $\overline{\mathrm{M}}_{0,5}$ consists of 10 irreducible curves isomorphic to $\mathbb{P}^{1}$. These are denoted by $D_{I}$, where $I \subseteq[5]$ is a subset of size 2. Moreover, two distinct boundary divisors $D_{I}, D_{J}$ intersect if and only if $I \subseteq J^{c}$. For $i \in[5]$, the Kapranov's map $\sigma_{i}$ contracts the boundary divisors $D_{I}$ such that $i \in I$, and away from these divisors $\sigma_{i}$ is an isomorphism. Given this description, it is clear that for all $x \in \overline{\mathrm{M}}_{0,5}$, there exists an open subset $U \subseteq \overline{\mathrm{M}}_{0,5}$ containing $x$ and $i \in[5]$ such that $\left.\sigma_{i}\right|_{U}$ is an isomorphism onto its image. This implies that the product of Kapranov's maps $\overline{\mathrm{M}}_{0,5} \rightarrow \prod_{i=1}^{5} \mathbb{P}^{2}$ is a closed embedding.

Remark 4.3. The product of Kapranov's maps $\overline{\mathrm{M}}_{0,8} \rightarrow \prod_{i=1}^{8} \mathbb{P}^{5}$ is not injective. Indeed, the curve $C=D_{12} \cap D_{34} \cap D_{56} \cap D_{78} \subseteq \overline{\mathrm{M}}_{0,8}$ is contracted by $\sigma_{1}$ because $\sigma_{1}\left(D_{12}\right)$ is a point. Analogously, $\sigma_{2}, \ldots, \sigma_{8}$ contract $C$. Hence, the product of Kapranov's maps contracts $C$.

Remark 4.4. We have a commutative diagram of closed embeddings

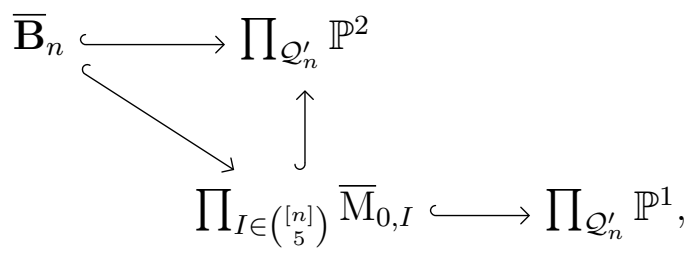

and we can embed $\prod_{\mathcal{Q}_{n}^{\prime}} \mathbb{P}^{1} \hookrightarrow \prod_{\mathcal{Q}_{n}} \mathbb{P}^{1}$ using a strategy analogous to the proof of Lemma 4.1 . In conclusion, we can view $\overline{\mathbf{B}}_{n}$ as a closed subvariety of $\prod_{\mathcal{Q}_{n}} \mathbb{P}^{1}$ and the embedding can be interpreted as follows: given an ordered quintuple $v \in \mathcal{Q}_{n}$, define $\mathbf{B}_{n} \rightarrow \mathbb{P}^{1}$ by sending $\left(p_{1}, \ldots, p_{n}\right)$ to the cross-ratio of four points in $\mathbb{P}^{1}$ obtained by projecting $p_{v_{1}}, \ldots, p_{v_{4}}$ from $p_{v_{5}}$. As a consequence of this discussion, $\overline{\mathbf{B}}_{5} \cong \overline{\mathrm{M}}_{0,5} \cong \overline{\mathrm{X}}(3,5)$.

\section{Mustafin Joins: General THEORY}

Mustafin joins were defined in the Introduction. Here we collect some basic facts.

Remark 5.1 ([CHSW11, $\S 2])$. Let $\Sigma=\left\{L_{1}, \ldots, L_{s}\right\} \subseteq \mathfrak{B}_{3}^{0}$. Fix a $K$-basis $e_{1}, e_{2}, e_{3}$ for $K^{3}$. For each $j=1, \ldots, s$, let $L_{j}=f_{1 j} R+f_{2 j} R+f_{3 j} R$ and let $g_{j} \in \mathrm{GL}_{3}(K)$ be the matrix with columns $f_{1 j}, f_{2 j}, f_{3 j}$. Denote by $X$ the matrix $\left(x_{i j}\right)_{1 \leq i \leq 3,1 \leq j \leq s}$, where we interpret the $j$-th column as homogeneous coordinates on the $j$-th copy of $\mathbb{P}^{2}$. Let $g(X)$ be the matrix obtained by applying $g_{j}$ to the $j$-th column of $X$ for all $j$. Then $\mathbb{P}(\Sigma)$ is isomorphic to the subscheme of $\left(\mathbb{P}_{R}^{2}\right)^{s}$ cut out by the multihomogeneous ideal $I_{2}(g(X)) \cap R[X]$, where $I_{2}$ denotes the ideal generated by the $2 \times 2$ minors. For example, let $\Sigma=\left\{\left[L_{1}\right],\left[L_{2}\right]\right\}$, where

$$
L_{1}=e_{1} R+e_{2} R+e_{3} R, L_{2}=t e_{1} R+e_{2} R+e_{3} R \text {. }
$$

The ideal of $\mathbb{P}(\Sigma) \subseteq \mathbb{P}\left(L_{1}\right) \times_{R} \mathbb{P}\left(L_{2}\right)$ is generated by the $2 \times 2$ minors of the matrix

$$
\left(\begin{array}{ll}
x_{11} & t x_{12} \\
x_{21} & x_{22} \\
x_{31} & x_{32}
\end{array}\right) .
$$


The special fiber $\mathbb{P}(\Sigma)_{\mathbb{k}}$ is defined by the following equations:

$$
x_{11} x_{22}=0, x_{11} x_{32}=0, x_{21} x_{32}-x_{31} x_{22}=0 .
$$

Its irreducible components are given by $V\left(x_{22}, x_{32}\right) \cong \mathbb{P}^{2}$ and $V\left(x_{11}, x_{21} x_{32}-x_{31} x_{22}\right) \cong \mathbb{F}_{1}$. These are glued along a line in $\mathbb{P}^{2}$ and the exceptional divisor in $\mathbb{F}_{1}$ as shown in Figure 1 .

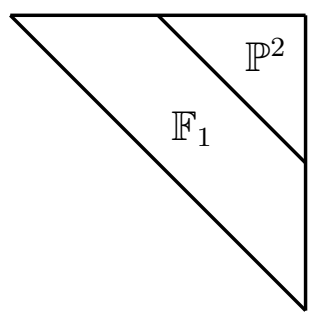

Figure 1. Central fiber of the Mustafin join $\mathbb{P}(\Sigma)$ in Remark 5.1 .

Remark 5.2. For a lattice $L$ we can define $\mathbb{P}^{\vee}(L)$ as $\operatorname{Proj}(\operatorname{Sym} L)$, and given a finite subset $\Sigma \subseteq \mathfrak{B}_{3}^{0}$, we can define $\mathbb{P}^{\vee}(\Sigma)$ accordingly. In general, the Mustafin joins $\mathbb{P}(\Sigma)$ and $\mathbb{P}^{\vee}(\Sigma)$ have non-isomorphic central fibers. For instance, consider $\Sigma=\left\{e_{1} R+e_{2} R+e_{3} R, t e_{1} R+\right.$ $\left.e_{2} R+t^{2} e_{3} R, e_{1} R+t e_{2} R+t^{2} e_{3} R\right\}$. Then $\mathbb{P}(\Sigma)_{\mathrm{k}}$ can be found in CHSW11, Figure 6 (iii), first picture], and $\mathbb{P}^{\vee}(\Sigma)_{\mathbb{k}}$ is in [CHSW11, Figure 6 (i), first picture].

Remark 5.3. An apartment $A \subseteq \mathfrak{B}_{3}^{0}$ corresponding to a basis $e_{1}, e_{2}, e_{3}$ of $K^{3}$ is the subset consisting of all lattice classes of the form

$$
\left[t^{\alpha} e_{1} R+t^{\beta} e_{2} R+t^{\gamma} e_{3} R\right],
$$

for $\alpha, \beta, \gamma \in \mathbb{Z}$. Given a finite subset $\Sigma \subseteq A$, the central fiber of the Mustafin join $\mathbb{P}(\Sigma)$ can be computed as follows [CHSW11, §4]. The apartment $A$ is identified with the tropical torus $\mathbb{R}^{3} / \mathbb{R} \mathbf{1}$, where $\mathbf{1}=(1,1,1)$, under the following bijection:

$$
\left[t^{\alpha} e_{1} R+t^{\beta} e_{2} R+t^{\gamma} e_{3} R\right] \mapsto(-\alpha,-\beta,-\gamma) .
$$

To each point $p \in \mathbb{R}^{3} / \mathbb{R} \mathbf{1}$, one can associate a tropical line (a spider with three legs)

$$
\ell_{p}=\left\{v \in \mathbb{R}^{3} / \mathbb{R} \mathbf{1} \mid \max _{i=1,2,3}\left\{v_{i}-p_{i}\right\} \text { is achieved at least twice }\right\} .
$$

Under the identification $A=\mathbb{R}^{3} / \mathbb{R} \mathbf{1}$, a lattice class $[L] \in \Sigma$ determines a tropical line $\ell_{[L]}$. The union of the bounded regions determined by the tropical lines $\ell_{[L]},[L] \in \Sigma$, gives a tropical polytope $P_{\Sigma}$, which is the min-convex hull of $\Sigma$. Consider the set of points $p \in P_{\Sigma}$ which correspond to a lattice class in $\Sigma$, or $p$ is the intersection of at least two tropical lines $\ell_{[L]},[L] \in \Sigma$. Such a point $p$ determines a projective toric variety whose polytope has edges orthogonal to the rays generating from $p$. Gluing all these polytopes we obtain a regular mixed polyhedral subdivision of $m \Delta_{2}$, where $m=|\Sigma|$ and $\Delta_{2}$ is the standard 2-dimensional simplex, which determines the central fiber of $\mathbb{P}(\Sigma)$. We illustrate this procedure in Figure 2. 

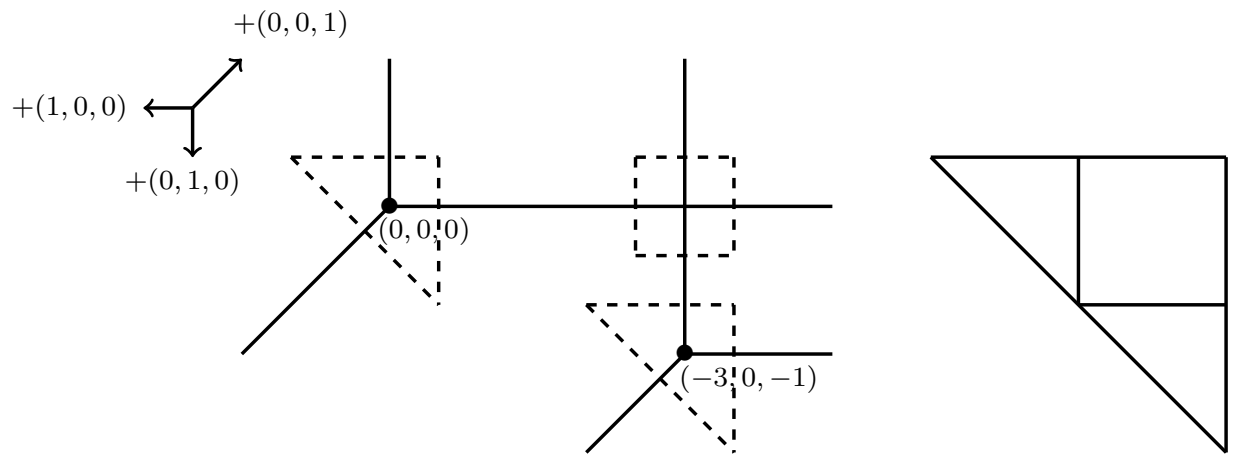

FiguRE 2. Illustration of the procedure described in Remark 5.3 to compute $\mathbb{P}(\Sigma)_{\mathbb{k}}$, where $\Sigma=\left\{\left[e_{1} R+e_{2} R+e_{3} R\right],\left[t^{3} e_{1} R+e_{2} R+t e_{3} R\right]\right\}$.

Definition 5.4. Let $\left[L_{0}\right],[L] \in \mathfrak{B}_{3}^{0}$. Let $z \in \mathbb{Z}$ be a unique integer such that $t L_{0} \subseteq t^{z} L$, but $L_{0} \nsubseteq t^{z} L$. Following [CHSW11, §5], we define a linear subspace induced by $[L]$ as

$$
W_{L_{0}}(L)=\mathbb{P}\left(\left(t^{z} L \cap L_{0}\right) / t L_{0}\right) \subseteq \mathbb{P}\left(L_{0}\right)_{\mathbb{k}} .
$$

Remark 5.5. Let $\Sigma=\left\{\left[L_{1}\right],\left[L_{2}\right]\right\}, L_{1} \neq L_{2}$. Consider the diagram

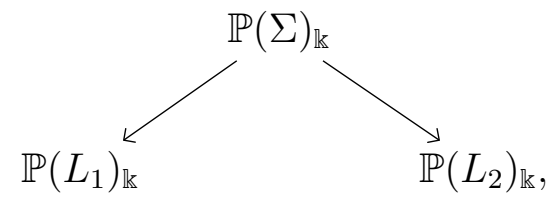

where the morphisms from $\mathbb{P}(\Sigma)_{\mathbb{k}} \subseteq \mathbb{P}\left(L_{1}\right)_{\mathbb{k}} \times \mathbb{P}\left(L_{2}\right)_{\mathbb{k}}$ are induced by the usual projections. There are two options for the central fiber $\mathbb{P}(\Sigma)_{\mathbb{k}}$ :

(1) $\mathbb{P}(\Sigma)_{\mathbb{k}}$ is the gluing of $\mathbb{P}^{2}$ and $\mathbb{F}^{1}$ along a line and the exceptional divisor, respectively;

(2) $\mathbb{P}(\Sigma)_{\mathbb{k}}$ is the union of two copies of $\mathbb{P}^{2}$ and $\mathbb{F}_{0}$. Each $\mathbb{P}^{2}$ is glued along a line to a ruling of $\mathbb{F}_{0}$, these two rulings intersect.

A comprehensive list of possibilities for the induced linear subspaces $W_{L_{1}}\left(L_{2}\right) \subseteq \mathbb{P}\left(L_{1}\right)_{\mathbb{k}}$ and $W_{L_{2}}\left(L_{1}\right) \subseteq \mathbb{P}\left(L_{2}\right)_{\mathbb{k}}$ is shown in Figure 3, where $W_{L_{1}}\left(L_{2}\right)$ and $W_{L_{2}}\left(L_{1}\right)$ are the images of appropriate irreducible components of $\mathbb{P}(\Sigma)_{\mathbb{k}}$ in $\mathbb{P}\left(L_{1}\right)_{\mathbb{k}}$ and $\mathbb{P}\left(L_{1}\right)_{\mathbb{k}}$, respectively. To prove these claims, we can assume that $\left[L_{1}\right],\left[L_{2}\right]$ lie in the same apartment Wer01, Proposition 4.11]

$$
L_{1}=e_{1} R+e_{2} R+e_{3} R, L_{2}=t^{\alpha} e_{1} R+t^{\beta} e_{2} R+t^{\gamma} e_{3} R,
$$

for some $K$-basis $e_{1}, e_{2}, e_{3}$ of $K^{3}$. If two among $\{\alpha, \beta, \gamma\}$ are equal, then without loss of generality $0=\alpha=\beta \neq \gamma$. In this case, $\mathbb{P}(\Sigma)_{\mathbb{k}}$ is as in (1) above. Moreover,

- If $\gamma<0$, then $W_{L_{1}}\left(L_{2}\right)$ is point, and $W_{L_{2}}\left(L_{1}\right)$ is a line;

- If $\gamma>0$, then $W_{L_{1}}\left(L_{2}\right)$ is a line, and $W_{L_{2}}\left(L_{1}\right)$ is a point.

If $\alpha, \beta, \gamma$ are all distinct, then without loss of generality $0=\alpha<\beta<\gamma$. Then $\mathbb{P}(\Sigma)_{\mathbb{k}}$ is as in $(2)$ and the induced linear spaces $W_{L_{1}}\left(L_{2}\right)$ and $W_{L_{2}}\left(L_{1}\right)$ are both lines. 

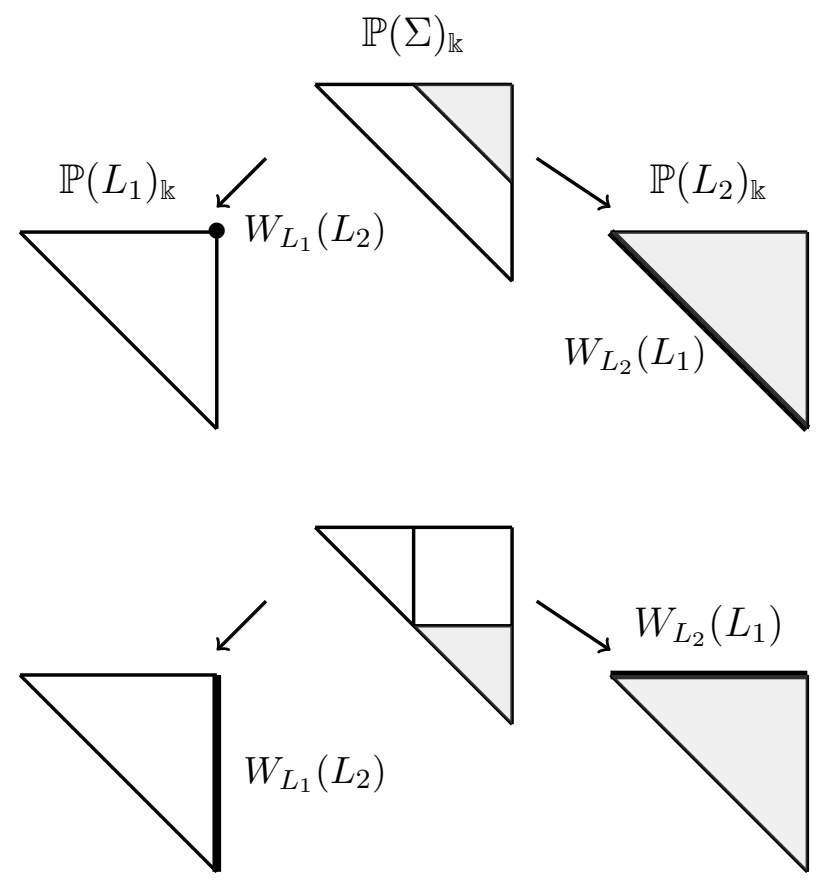

Figure 3 . The linear subspaces $W_{L_{1}}\left(L_{2}\right)$ and $W_{L_{2}}\left(L_{1}\right)$ in relation to $\mathbb{P}(\Sigma)_{\mathbb{k}}$.

Definition 5.6 ([CHSW11]). An irreducible component of the central fiber $\mathbb{P}(\Sigma)_{\mathbb{k}}$ is primary if it projects birationally onto one of the $\mathbb{P}(L)_{\mathbb{k}}, L \in \Sigma$. Other components are secondary.

Lemma 5.7. Let $s=[v] \in \mathbb{P}^{2}(K)$ and let $\Sigma$ be a finite subset of $\mathfrak{B}_{3}^{0}$. We can write

$$
\Sigma=\left\{\left[L_{1}\right], \ldots,\left[L_{m}\right]\right\}
$$

for a unique choice of lattices if we assume that $v \in L_{j} \backslash t L_{j}$ for all $j$. Suppose that one of the lattices $L_{i_{0}} \subseteq L_{1} \cap \ldots \cap L_{m}$. Then $\bar{s}(0) \in \mathbb{P}(\Sigma)_{\mathbb{k}}$ is a smooth point contained in the primary component corresponding to $L_{i_{0}}$. In other words, $\mathbb{P}(\Sigma)$ is smooth along the section s.

Proof. For each $j \neq i_{0}$, fix an integer $z_{j}$ such that $t L_{i_{0}} \subseteq t^{z_{j}} L_{j} \backslash t^{z_{j}+1} L_{j}$. We have $z_{j}>0$. Otherwise, if $z_{j} \leq 0$, then $L_{i_{0}} \subseteq L_{j} \subseteq t^{z_{j}} L_{j}$, which gives a contradiction. By [CHSW11, §5], the primary component corresponding to $L_{i_{0}}$ is obtained by blowing up $\mathbb{P}\left(L_{i_{0}}\right)_{\mathbb{k}}$ along the proper linear subspaces $W_{L_{i_{0}}}\left(L_{j}\right)$ for $j \neq i_{0}$ (in particular, it is smooth). So, we would be done if we can show that the image of $v$ in $L_{i_{0}} / t L_{i_{0}}$ is not contained in $\left(t^{z_{j}} L_{j} \cap L_{i_{0}}\right) / t L_{i_{0}}$ for all $j \neq i_{0}$. But this is clear, because $t^{z_{j}} L_{j} \subseteq t L_{j}$ and $v \notin t L_{j}$ by hypothesis.

Finally, we study how the Mustafin join changes when we add a lattice to the configuration.

Lemma 5.8. Let $\Sigma=\left\{\left[L_{1}\right], \ldots,\left[L_{m}\right]\right\} \subseteq \mathfrak{B}_{3}^{0}$ and $\Sigma^{\prime}=\Sigma \cup\left\{\left[L_{0}\right]\right\}$. Let $\widetilde{C} \subseteq \mathbb{P}\left(\Sigma^{\prime}\right)_{\mathbb{k}}$ be the primary component corresponding to $\left[L_{0}\right]$. Let $\ell \subseteq \mathbb{P}\left(L_{0}\right)_{\mathbb{k}}$ be a line and denote by $\tilde{\ell}$ its strict transform in $\widetilde{C}$. Then $\widetilde{\ell}$ is contracted by the projection $\pi: \mathbb{P}\left(\Sigma^{\prime}\right) \rightarrow \mathbb{P}(\Sigma)$ if and only if $\ell$ intersects every linear subspace $W_{L_{0}}\left(L_{j}\right) \subseteq \mathbb{P}\left(L_{0}\right)_{\mathbb{k}}$. Otherwise, $\left.\pi\right|_{\tilde{\ell}}$ is injective. 
Proof. Suppose $\ell$ intersects every linear subspace $W_{L_{0}}\left(L_{j}\right)$. For every $j$, let $\Lambda=\left\{\left[L_{0}\right],\left[L_{j}\right]\right\}$, and consider the diagram $\mathbb{P}\left(L_{0}\right)_{\mathbb{k}} \leftarrow \mathbb{P}(\Lambda)_{\mathbb{k}} \rightarrow \mathbb{P}\left(L_{j}\right)_{\mathbb{k}}$. After inspecting all the possibilities for $\mathbb{P}(\Lambda)_{\mathbb{k}}$ (see Remark 5.5) and $W_{L_{0}}\left(L_{j}\right)$ (which is a point or a line), we can see that the image of $\tilde{\ell}$ in $\mathbb{P}\left(L_{j}\right)_{\mathbb{k}}$ of $\tilde{\ell}$ is a point. Since this is true for every $j, \pi$ contracts $\tilde{\ell}$.

Conversely, if $\ell$ does not intersect a linear space $W_{L_{0}}\left(L_{j}\right)$ for some $j$ then by considering the same diagram (where now we only have to consider the case where $W_{L_{0}}\left(L_{j}\right)$ is a point), we see that the image of $\tilde{\ell}$ in $\mathbb{P}\left(L_{j}\right)_{\mathbb{k}}$ is not contracted, in fact $\left.\pi\right|_{\tilde{\ell}}$ is injective.

Remark 5.9. An almost identical statement appears in [CHSW11, Lemma 5.10, Proposition 5.11], but with stronger hypotheses: we do not require that $\left.\pi\right|_{\widetilde{C}}$ is birational onto its image.

\section{Stable lattices of ARCS AND their Mustafin Joins}

Here we focus on Mustafin joins for arcs considered in [GP91]. Consider $K$-points

$$
a_{1}, \ldots, a_{n} \in \mathbb{P}^{2}(K)
$$

in general linear position, i.e. an $\operatorname{arc} \mathbf{a}=\left(a_{1}, \ldots, a_{n}\right)$ : $\operatorname{Spec}(K) \rightarrow \mathbf{B}_{n}$. We denote by $\Sigma_{\mathbf{a}}$ the subset of $\mathfrak{B}_{3}^{0}$ of stable lattice classes with respect to a. Recall from the introduction that a lattice $\Lambda$ is stable if at least four of the limits $\bar{a}_{1}^{L}(0), \ldots, \bar{a}_{n}^{L}(0)$ in the central fiber $\mathbb{P}(L)_{\mathbb{k}} \subseteq \mathbb{P}(L)$ are in general linear position. There exists a unique lattice class stabilizing any given quadruple [KT06], although the same lattice can stabilize several quadruples. The Mustafin join $\mathbb{P}\left(\Sigma_{\mathbf{a}}\right)$ has extra structure, namely $n$ sections $\bar{a}_{1}, \ldots, \bar{a}_{n}$ which are defined as follows. Every component $a_{i}$ can be viewed as a section of $\mathbb{P}\left(\Sigma_{\mathbf{a}}\right)_{K} \rightarrow \operatorname{Spec}(K)$ using an isomorphism $\mathbb{P}\left(\Sigma_{\mathbf{a}}\right)_{K} \cong \mathbb{P}_{K}^{2}$. By the valuative criterion of properness, $a_{i}$ admits a unique extension $\bar{a}_{i}: \operatorname{Spec}(R) \rightarrow \mathbb{P}\left(\Sigma_{\mathbf{a}}\right)$, which is the claimed section.

Lemma 6.1. The sections $\bar{a}_{1}, \ldots, \bar{a}_{n}$ of the Mustafin join $\mathbb{P}\left(\Sigma_{\mathbf{a}}\right)$ are pairwise disjoint.

Proof. It suffices to check the claim on the central fiber $\mathbb{P}\left(\Sigma_{\mathbf{a}}\right)_{\mathbb{k}}$. Let $1 \leq i<j \leq n$. Write $\Sigma_{\mathbf{a}}=\left\{L_{1}, \ldots, L_{m}\right\}$. By the definition of stable lattices, there exists $h \in\{1, \ldots, m\}$ such that $\bar{a}_{i}(0), \bar{a}_{j}(0) \in \mathbb{P}\left(L_{h}\right)_{\mathbb{k}}$ are distinct. Then from the commutativity of the following diagram:

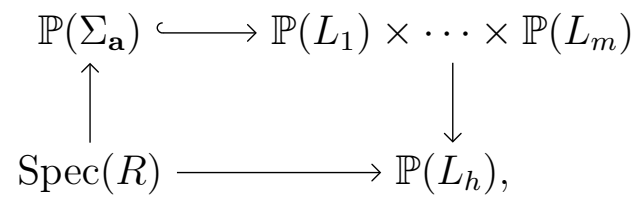

we can conclude that $\bar{a}_{i}(0), \bar{a}_{j}(0) \in \mathbb{P}\left(\Sigma_{\mathbf{a}}\right)_{k}$ are also distinct.

Example 6.2. Let $\left(a_{1}, \ldots, a_{5}\right)=\left([1: 0: 0],[0: 1: 0],[0: 0: 1],[1: 1: 1],\left[t^{2}: 1: t\right]\right)$. Then

$$
\Sigma_{\mathbf{a}}=\left\{\left[e_{1} R+e_{2} R+e_{3} R\right],\left[t^{2} e_{1} R+e_{2} R+t e_{3} R\right],\left[t e_{1} R+e_{2} R+t e_{3} R\right]\right\} .
$$

To prove this, we list all possible quadruples $i_{1}, \ldots, i_{4}$ and the corresponding stable lattice

$$
\{1234\}, \quad\{1345\} \quad e_{1} R+e_{2} R+e_{3} R,
$$


$\{1235\}$,
$\{2345\}$

$$
\begin{array}{r}
t^{2} e_{1} R+e_{2} R+t e_{3} R, \\
t e_{1} R+e_{2} R+t e_{3} R .
\end{array}
$$

Using the procedure of Remark 5.3 , the central fiber $\mathbb{P}\left(\Sigma_{\mathbf{a}}\right)_{\mathbb{k}}$ with sections is shown in Figure 4
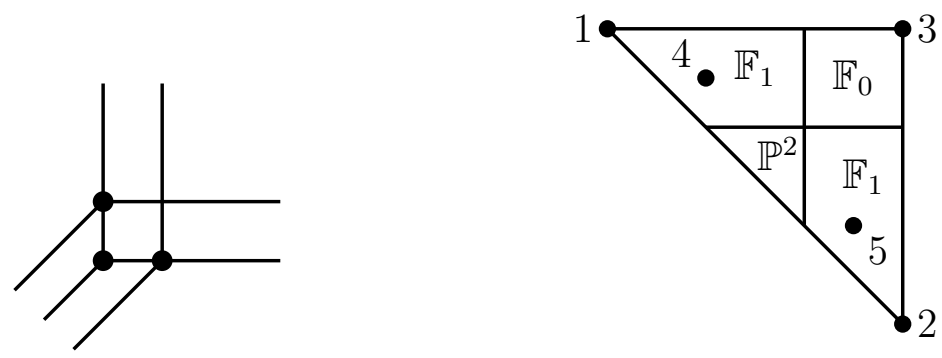

FiguRE 4 . The lattice classes in $\Sigma_{\mathbf{a}}$ (in the apartment corresponding to the basis $\left.e_{1}, e_{2}, e_{3}\right)$ and the central fiber of the Mustafin join $\mathbb{P}\left(\Sigma_{\mathbf{a}}\right)$ of Example 6.2.

In the remainder of $\$ 6$ we will show that the Mustafin join of stable lattices $\mathbb{P}\left(\Sigma_{\mathbf{a}}\right) \rightarrow$ $\operatorname{Spec}(R)$ is smooth along the sections $\bar{a}_{1}, \ldots, \bar{a}_{n}$, i.e. $\bar{a}_{1}(0), \ldots, \bar{a}_{n}(0)$ are smooth points on the central fiber $\mathbb{P}\left(\Sigma_{\mathbf{a}}\right)_{\mathrm{k}}$. This was claimed in [GP91, §4.2], but no proof was given. We fix vectors

$$
v_{1}, \ldots, v_{n} \in K^{3}
$$

such that $a_{i}=\left[v_{i}\right] \in \mathbb{P}^{2}(K)$ are in general linear position. After reordering, it suffices to prove this for the first section $\bar{a}_{1}$. We start by introducing canonical normalizations of vectors $v_{1}, \ldots, v_{n}$ as well as lattices $\Lambda$ for all lattice classes $[\Lambda]$ in the building.

Definition 6.3. We call a lattice $\Lambda$ normalized with respect to $v_{1}$ if $v_{1} \in \Lambda \backslash t \Lambda$.

Lemma-Definition 6.4. For any quadruple $I \subseteq\{1, \ldots, n\}$, write $\sum_{i \in I} k_{i} v_{i}=0, k_{i} \in K \backslash\{0\}$. The lattice $L_{I}:=\sum_{i \in I} k_{i} v_{i} R$ stabilizes the quadruple $\left\{a_{i} \mid i \in I\right\}$. After rescaling the coefficients $k_{i}$, we can assume that all the lattices $L_{I}$ are normalized with respect to $v_{1}$.

Proof. Since the vectors $\left\{v_{i} \mid i \in I\right\}$ are linearly dependent, we can write $\sum_{i \in I} k_{i} v_{i}=0$ for some $k_{i} \in K$ not all equal to 0 . In fact $k_{i} \neq 0$ for all $i$ because any three vectors are linearly independent. In the basis $k_{1} v_{1}, k_{2} v_{2}, k_{3} v_{3}$ of $L_{I}$, the limits $a_{i}(0), i \in I$, are [1:0:0], [0:1:0], $[0: 0: 1],[1: 1: 1]$, respectively. Thus $L_{I}$ stabilizes the quadruple $\left\{a_{i} \mid i \in I\right\}$.

Definition 6.5. Fix an isomorphism $\varphi: \bigwedge^{3} K^{3} \cong K$ and let val: $K \backslash\{0\} \rightarrow \mathbb{Z}$ be the usual valuation. For three linearly independent vectors $u, v, w$ in $K^{3}$, consider the log volume

$$
\|u, v, w\|=\operatorname{val}(\varphi(u \wedge v \wedge w)) .
$$

Lemma-Definition 6.6. We can rescale $v_{2}, \ldots, v_{n}$ uniquely by $t^{p_{2}}, \ldots, t^{p_{n}}$ so that

(1) For any quadruple $1, i, j, k$, we have $\left\|v_{i}, v_{j}, v_{k}\right\| \geq\left\|v_{1}, v_{j}, v_{k}\right\|$; 
(2) For all $i \neq 1$, there exists a quadruple $1, i, j, k$ such that $\left\|v_{i}, v_{j}, v_{k}\right\|=\left\|v_{1}, v_{j}, v_{k}\right\|$. In this case we say that $v_{1}, \ldots, v_{n}$ are normalized with respect to $v_{1}$.

Proof. Let $i \in\{2, \ldots, n\}$ and define $p_{i}$ to be the least integer such that

$$
\left\|t^{p_{i}} v_{i}, v_{j}, v_{k}\right\|=p_{i}+\left\|v_{i}, v_{j}, v_{k}\right\| \geq\left\|v_{1}, v_{j}, v_{k}\right\|
$$

for all distinct $j, k \neq 1, i$. Note that the inequality depends not on $v_{j}, v_{k}$ but only on the corresponding points $a_{j}, a_{k} \in \mathbb{P}^{2}(K)$. By minimality of $p_{i}$, equality is achieved for at least one pair $j, k$. Therefore, $t^{p_{2}} v_{2}, \ldots, t^{p_{n}} v_{n}$ satisfy properties (1) and (2).

Lemma 6.7. Assume $v_{1}, \ldots, v_{n}$ are normalized with respect to $v_{1}$. If $1 \in I \in\left(\begin{array}{c}{[n]} \\ 4\end{array}\right)$, then

$$
\Lambda_{I}=\left\{u \in K^{3} \mid\left\|u, v_{j}, v_{k}\right\| \geq\left\|v_{1}, v_{j}, v_{k}\right\| \text { for all } j, k \in I \backslash\{1\}\right\}
$$

is a lattice normalized with respect to $v_{1}$ and stabilizing the quadruple $a_{i}, i \in I$.

Proof. Assume $I=\{1, \ldots, 4\}$. Write $v_{1}=c_{2} v_{2}+c_{3} v_{3}+c_{4} v_{4}$ for $c_{2}, c_{3}, c_{4} \in K$. By LemmaDefinition 6.4 the lattice $L_{I}=c_{2} v_{2} R+c_{3} v_{3} R+c_{4} v_{4} R$ stabilizes $a_{1}, \ldots, a_{4}$. By Cramer's rule,

$$
c_{2}=\frac{\varphi\left(v_{1} \wedge v_{3} \wedge v_{4}\right)}{\varphi\left(v_{2} \wedge v_{3} \wedge v_{4}\right)}, c_{3}=-\frac{\varphi\left(v_{1} \wedge v_{2} \wedge v_{4}\right)}{\varphi\left(v_{2} \wedge v_{3} \wedge v_{4}\right)}, c_{4}=\frac{\varphi\left(v_{1} \wedge v_{2} \wedge v_{3}\right)}{\varphi\left(v_{2} \wedge v_{3} \wedge v_{4}\right)} .
$$

Let $u \in L_{I}$. Then $u=r_{2} c_{2} v_{2}+r_{3} c_{3} v_{3}+r_{4} c_{4} v_{4}$ for some $r_{2}, r_{3}, r_{4} \in R$. By Cramer's rule,

$$
r_{2} c_{2}=\frac{\varphi\left(u \wedge v_{3} \wedge v_{4}\right)}{\varphi\left(v_{2} \wedge v_{3} \wedge v_{4}\right)}, r_{3} c_{3}=-\frac{\varphi\left(u \wedge v_{2} \wedge v_{4}\right)}{\varphi\left(v_{2} \wedge v_{3} \wedge v_{4}\right)}, r_{4} c_{4}=\frac{\varphi\left(u \wedge v_{2} \wedge v_{3}\right)}{\varphi\left(v_{2} \wedge v_{3} \wedge v_{4}\right)} .
$$

Since $\operatorname{val}\left(r_{i} c_{i}\right) \geq \operatorname{val}\left(c_{i}\right),(2)$ and (3) imply that $\left\|u, v_{j}, v_{k}\right\| \geq\left\|v_{1}, v_{j}, v_{k}\right\|$. Thus $L_{I} \subseteq \Lambda_{I}$. For the other containment, let $u \in \Lambda_{I}$ and write $u=d_{2} v_{2}+d_{3} v_{3}+d_{4} v_{4}$ for some $d_{2}, d_{3}, d_{4} \in K$. Since $\left\|u, v_{j}, v_{k}\right\| \geq\left\|v_{1}, v_{j}, v_{k}\right\|$ for all $j, k \in I \backslash\{1\}$, we have $\operatorname{val}\left(d_{i}\right) \geq \operatorname{val}\left(c_{i}\right)$ for all $i=2,3,4$ by Cramer's rule. Thus $d_{i}=r_{i} c_{i}$ for some $r_{i} \in R$, implying that $u \in L_{I}$.

Corollary 6.8. Assume $v_{1}, \ldots, v_{n}$ are normalized with respect to $v_{1}$. Write $\Sigma_{\mathbf{a}}=\left\{\left[L_{j}\right]\right\}$, where every $L_{j}$ is normalized with respect to $v_{1}$. Then

$$
\Lambda=\bigcap_{\left[L_{j}\right] \in \Sigma_{\mathbf{a}}} L_{j}=\left\{u \in K^{3} \mid\left\|u, v_{j}, v_{k}\right\| \geq\left\|v_{1}, v_{j}, v_{k}\right\| \text { for all } j, k \in[n] \backslash\{1\}\right\} .
$$

Proof. We use the notation of Lemma-Definition 6.4. By Lemma 6.7, it suffices to prove that $\bigcap_{I \in\left(\begin{array}{c}{[n]} \\ 4\end{array}\right)} L_{I}=\bigcap_{1 \in I \in\left(\begin{array}{c}{[n]} \\ 4\end{array}\right)} L_{I}$.

Let $I \in\left(\begin{array}{c}{[n]} \\ 4\end{array}\right), 1 \notin I$. It suffices to show that there exists $J \in\left(\begin{array}{c}{[n]} \\ 4\end{array}\right)$ such that $1 \in J$ and $L_{J} \subseteq L_{I}$. By assumption, $v_{1} \in L_{I}$, hence we can write

$$
v_{1}=\sum_{i \in I} r_{i} k_{i} v_{i}
$$

for some $r_{i} \in R$. Let $j \in I$ be arbitrary and write $k_{j} v_{j}=\sum_{i \in I \backslash\{j\}}\left(-k_{i}\right) v_{i}$. By substituting into the previous expression for $v_{1}$ we obtain

$$
v_{1}=\sum_{i \in I \backslash\{j\}}\left(r_{i}-r_{j}\right) k_{i} v_{i} .
$$


Let $\Lambda=\sum_{i \in I \backslash\{j\}}\left(r_{i}-r_{j}\right) k_{i} v_{i} R$. Since $\Lambda$ is contained in $L_{I}$, it suffices to show that $L_{(I \backslash\{j\}) \cup\{1\}}=\Lambda$. By Lemma-Definition 6.4 and (4), this is true up to rescaling, so we only have to show that $\Lambda$ is normalized with respect to $v_{1}$. By (4) we have that $v_{1} \in \Lambda$, so we just need to show that $t^{-1} v_{1} \notin \Lambda$. But this is true because $t^{-1} v_{1} \notin L_{I}$.

Theorem 6.9. Let $\mathbf{a}=\left(a_{1}, \ldots, a_{n}\right) \in\left(\mathbb{P}^{2}\right)^{n}(K)$ be in general linear position. Then the Mustafin join $\mathbb{P}\left(\Sigma_{\mathbf{a}}\right) \rightarrow \operatorname{Spec}(R)$ is smooth along the $n$ disjoint sections $\bar{a}_{1}, \ldots, \bar{a}_{n}$.

Proof. We need to show that the sections determine smooth points of the central fiber. Up to permuting the indices, it suffices to show that $\bar{a}_{1}(0) \in \mathbb{P}\left(\Sigma_{a}\right)_{\mathbb{k}}$ is a smooth point. Write

$$
a_{1}=\left[v_{1}\right], \ldots, a_{n}=\left[v_{n}\right]
$$

where $v_{1}, \ldots, v_{n}$ are normalized with respect to $v_{1}$ (Lemma-Definition 6.6). Using Lemma 6.7. we can write the set of stable lattices with respect to a as

$$
\Sigma_{\mathbf{a}}=\left\{\left[\Lambda_{I}\right] \mid I \in\left(\begin{array}{c}
{[n]} \\
4
\end{array}\right)\right\},
$$

where $\Lambda_{I}$ is normalized with respect to $v_{1}$ and stabilizes the quadruple $a_{i}, i \in I$. If the set $\Sigma_{\mathbf{a}}$ has a minimal element with respect to inclusion of lattices then we are done by Lemma 5.7 .

Suppose there is no minimal element in $\Sigma_{\mathbf{a}}$ and consider $\Lambda$ of Corollary 6.8, the intersection of all the $\Lambda_{I}$ 's. Then $[\Lambda] \notin \Sigma_{\mathbf{a}}$. Define $\Sigma_{\mathbf{a}}^{\prime}=\Sigma_{\mathbf{a}} \cup\{[\Lambda]\}$. Let $\widetilde{C}$ be the primary component of $\mathbb{P}\left(\Sigma_{\mathbf{a}}^{\prime}\right)_{\mathbb{k}}$ corresponding to $[\Lambda]$. Recall that $\widetilde{C}$ is the blow up of $\mathbb{P}(\Lambda)_{\mathbb{k}}$ at the linear subspaces $W_{\Lambda}\left(\Lambda_{I}\right)$ for $I \in\left(\begin{array}{c}{[n]} \\ 4\end{array}\right)$ (see CHSW11, Proposition 5.6]). By Lemma 5.7, $\bar{a}_{i}(0) \in \mathbb{P}\left(\Sigma_{\mathbf{a}}^{\prime}\right)_{\mathbb{k}}$ is a smooth point, and $\widetilde{C}$ is the primary component containing $\bar{a}_{1}(0) \in \mathbb{P}\left(\Sigma_{\mathbf{a}}^{\prime}\right)_{\mathbb{k}}$. In particular, $\bar{a}_{1}(0) \in \mathbb{P}(\Lambda)_{\mathbb{k}}$ is not contained in any of the linear subspaces $W_{\Lambda}\left(\Lambda_{I}\right), I \in\left(\begin{array}{c}{[n]} \\ 4\end{array}\right)$.

We have a morphism $\pi: \mathbb{P}\left(\Sigma_{\mathbf{a}}^{\prime}\right) \rightarrow \mathbb{P}\left(\Sigma_{\mathbf{a}}\right)$. We will show later on in the proof that $\left.\pi\right|_{\widetilde{C}}$ is birational onto its image. For now, let us assume it. If $\ell \subseteq \mathbb{P}(\Lambda)_{\mathbb{k}}$ is a line, then we denote by $\tilde{\ell} \subseteq \widetilde{C}$ its strict transform. By [CHSW11, Lemma 5.10], $\widetilde{\ell}$ is contracted by $\pi$ if and only if $\ell$ intersects all linear subspaces $W_{\Lambda}\left(\Lambda_{I}\right)$. Moreover, by [CHSW11, Proposition 5.11], the union of all contracted $\widetilde{\ell}^{\prime}$ s is the exceptional locus of $\left.\pi\right|_{\widetilde{C}}$. So it suffices to show that there is no line $\ell \subseteq \mathbb{P}(\Lambda)_{\mathbb{k}}$ such that $\bar{a}_{1}(0) \in \tilde{\ell}$ and $\ell$ intersects all the linear subspaces $W_{\Lambda}\left(\Lambda_{I}\right), I \in\left(\begin{array}{c}{[n]} \\ 4\end{array}\right)$. Arguing by contradiction, suppose $\ell$ exists. Since $\left.\pi\right|_{\widetilde{C}}$ is birational, there exist $\Lambda_{J}, \Lambda_{K}$ inducing two distinct points $W_{\Lambda}\left(\Lambda_{J}\right), W_{\Lambda}\left(\Lambda_{K}\right) \in \mathbb{P}(\Lambda)_{\mathbb{k}}$. These points lie on $\ell$. By Lemma 6.10, $W_{\Lambda}\left(\Lambda_{J}\right)=\bar{a}_{j}(0)$ and $W_{\Lambda}\left(\Lambda_{K}\right)=\bar{a}_{k}(0)$ for some sections $\bar{a}_{j}$ and $\bar{a}_{k}$. Therefore, $\bar{a}_{1}(0), \bar{a}_{j}(0), \bar{a}_{k}(0)$ are three distinct limit points on $\ell$. By Lemma 6.11, $\ell$ is a linear subspace of $\mathbb{P}(\Lambda)_{\mathbb{k}}$ induced by a stable lattice. But this is a contradiction, since we know that $\bar{a}_{1}(0) \in \mathbb{P}(\Lambda)_{\mathbb{k}}$ cannot lie on any of the linear spaces $W_{\Lambda}\left(\Lambda_{I}\right)$.

To conclude the proof, it remains to show that $\left.\pi\right|_{\widetilde{C}}$ is birational onto its image. For any $i \in$ $\{2, \ldots, n\}$, there is a quadruple $I=\{1, i, j, k\}$ such that $\left\|v_{i}, v_{j}, v_{k}\right\|=\left\|v_{1}, v_{j}, v_{k}\right\|$ (LemmaDefinition 6.6). We claim that $\bar{a}_{i}(0) \in \mathbb{P}(\Lambda)_{\mathbb{k}}$ is disjoint from $W_{\Lambda}\left(\Lambda_{I}\right)=\mathbb{P}\left(\left(t^{z_{I}} \Lambda_{I} \cap \Lambda\right) / t \Lambda\right)$, where $z_{I}$ is that unique integer such that $t \Lambda \subseteq t^{z_{I}} \Lambda_{I}$ and $\Lambda \nsubseteq t^{z_{I}} \Lambda_{I}$. Note that $z_{I}>0$. It is enough to show that $v_{i} \notin t^{z_{I}} \Lambda_{I} \subseteq t \Lambda_{I}$. Assume by contradiction that $v_{i} \in t \Lambda_{I}$. So $v_{i}=t u$, 
where $u \in K^{3}$ satisfies $\left\|u, v_{j}, v_{k}\right\| \geq\left\|v_{1}, v_{j}, v_{k}\right\|$ (see Lemma 6.7). Hence

$$
\left\|u, v_{j}, v_{k}\right\| \geq\left\|v_{1}, v_{j}, v_{k}\right\|=\left\|v_{i}, v_{j}, v_{k}\right\|=\left\|t u, v_{j}, v_{k}\right\|=1+\left\|u, v_{j}, v_{k}\right\|,
$$

which is impossible. Next we claim that $W_{\Lambda}\left(\Lambda_{I}\right)$ is a point. If not, then both $\bar{a}_{1}(0), \bar{a}_{i}(0) \in$ $\mathbb{P}(\Lambda)_{\mathbb{k}}$ lie outside the line $W_{\Lambda}\left(\Lambda_{I}\right)$. On the other hand, the limits $\bar{a}_{1}(0), \bar{a}_{i}(0) \in \mathbb{P}\left(\Lambda_{I}\right)_{\mathbb{k}}$ are distinct because $\Lambda_{I}$ stabilizes $a_{1}, a_{i}, a_{j}, a_{k}$. Analyzing both possible diagrams $\mathbb{P}(\Lambda)_{\mathbb{k}} \leftarrow$ $\mathbb{P}\left(\Lambda, \Lambda_{I}\right)_{\mathbb{k}} \rightarrow \mathbb{P}\left(\Lambda_{I}\right)_{\mathbb{k}}$ of Figure 3 , we arrive at a contradiction. Since $W_{\Lambda}\left(\Lambda_{I}\right)$ is a point, by Lemma 6.10 it is equal to $\bar{a}_{h}(0)$ for some $h \in[n] \backslash\{1\}$. Now repeat the same argument above with $i$ replaced by $h$ to find a second induced linear subspace which is a point distinct from $\bar{a}_{1}(0)$ and $W_{\Lambda}\left(\Lambda_{I}\right)$. By Lemma 5.8 , this implies that $\left.\pi\right|_{\widetilde{C}}$ is birational onto its image.

Lemma 6.10. Let $\mathbf{a}=\left(a_{1}, \ldots, a_{n}\right) \in\left(\mathbb{P}^{2}\right)^{n}(K)$ be in general linear position and let $\left[L_{0}\right] \in \mathfrak{B}_{3}^{0}$ be arbitrary. Let $[L] \in \Sigma_{\mathbf{a}}$ be a stable lattice class and assume that the linear subspace $W_{L_{0}}(L) \subseteq \mathbb{P}\left(L_{0}\right)_{\mathbb{k}}$ is a point. Then there exist distinct $i, j \in[n]$ such that

$$
\bar{a}_{i}(0)=W_{L_{0}}(L)=\bar{a}_{j}(0) .
$$

Proof. Up to relabeling the indices, assume that $a_{1}, \ldots, a_{4}$ is the quadruple stabilized by $[L]$. We show that at least two among $\bar{a}_{1}(0), \ldots, \bar{a}_{4}(0) \in \mathbb{P}\left(L_{0}\right)_{\mathbb{k}}$ equal $W_{L_{0}}(L)$. Let $\Sigma=\left\{\left[L_{0}\right],[L]\right\}$ and consider the diagram $\mathbb{P}\left(L_{0}\right)_{\mathbb{k}} \leftarrow \mathbb{P}(\Sigma)_{\mathbb{k}} \rightarrow \mathbb{P}(L)_{\mathbb{k}}$. Since $W_{L_{0}}(L)$ is a point, by Remark 5.5. $\mathbb{P}(\Sigma)_{\mathbb{k}}$ is isomorphic to the gluing of $\mathbb{P}^{2}$ and $\mathbb{F}_{1}$ along a line and the exceptional divisor respectively. The morphism $\mathbb{P}(\Sigma)_{\mathbb{k}} \rightarrow \mathbb{P}\left(L_{0}\right)_{\mathbb{k}}$ is the blow up at the point $W_{L_{0}}(L)$, and the morphism $\mathbb{P}(\Sigma)_{\mathbb{k}} \rightarrow \mathbb{P}(L)_{\mathbb{k}}$ contracts $\mathbb{F}_{1}$ along the ruling. Therefore, if no two points among $\bar{a}_{1}(0), \ldots, \bar{a}_{4}(0) \in \mathbb{P}\left(L_{0}\right)_{\mathbb{k}}$ are equal to $W_{L_{0}}(L)$, then $\bar{a}_{1}(0), \ldots, \bar{a}_{4}(0) \in \mathbb{P}(L)_{\mathbb{k}}$ would not be in general linear position.

Lemma 6.11. Let $\mathbf{a}=\left(a_{1}, \ldots, a_{n}\right) \in\left(\mathbb{P}^{2}\right)^{n}(K)$ be in general linear position and let $\left[L_{0}\right] \in \mathfrak{B}_{3}^{0}$ be arbitrary. If three limit points $\bar{a}_{i}(0), \bar{a}_{j}(0), \bar{a}_{k}(0) \in \mathbb{P}\left(L_{0}\right)_{\mathbb{k}}$ are distinct and contained in $a$ line $\ell$, then any $[L] \in \Sigma_{\mathbf{a}}$ stabilizing $a_{i}, a_{j}, a_{k}$ has the property that $W_{L_{0}}(L)=\ell$.

Proof. Let $[L] \in \Sigma_{\mathbf{a}}$ be any stable lattice class stabilizing a quadruple that includes $a_{i}, a_{j}, a_{k}$. Define $\Sigma=\left\{\left[L_{0}\right],[L]\right\}$ and consider the diagram $\mathbb{P}\left(L_{0}\right)_{\mathbb{k}} \leftarrow \mathbb{P}(\Sigma)_{\mathbb{k}} \rightarrow \mathbb{P}(L)_{\mathbb{k}}$, where in $\mathbb{P}\left(L_{0}\right)_{\mathbb{k}}$ the three limit points $\bar{a}_{i}(0), \bar{a}_{j}(0), \bar{a}_{k}(0)$ are aligned, but the same limits in $\mathbb{P}(L)_{\mathbb{k}}$ are in general linear position. By Remark 5.5, there are two possibilities for $\mathbb{P}(\Sigma)_{\mathbb{k}}$. Considering all the possible ways the three aligned limit points in $\mathbb{P}\left(L_{0}\right)_{\mathbb{k}}$ can be related with respect to $W_{L_{0}}(L)$, and considering that these limits are in general linear position in $\mathbb{P}(L)_{\mathbb{k}}$, we see that $W_{L_{0}}(L)$ is a line and $\bar{a}_{i}(0), \bar{a}_{j}(0), \bar{a}_{k}(0) \in W_{L_{0}}(L)$.

\section{Universal MUSTAFin JOIN FOR ARCS}

Example 7.1. We start with a counter-example to the claim [GP91, §4, Proposition 1] that $\overline{\mathbf{B}}_{n}$ is the moduli space of Mustafin joins for arcs. Define $\operatorname{arcs} \mathbf{a}, \mathbf{b}: \operatorname{Spec}(K) \rightarrow \mathbf{B}_{6}$,

$$
\begin{aligned}
& \mathbf{a}(t)=\left([1: 0: 0],[0: 1: 0],[0: 0: 1],[1: 1: 1],\left[1: t: t^{2}\right],\left[t: 1: t^{2}\right]\right), \\
& \mathbf{b}(t)=\left([1: 0: 0],[0: 1: 0],[0: 0: 1],[1: 1: 1],\left[1: t^{2}: t^{3}\right],\left[t: 1: t^{3}\right]\right) .
\end{aligned}
$$


We claim that the limit points $\overline{\mathbf{a}}(0), \overline{\mathbf{b}}(0) \in \overline{\mathbf{B}}_{6}$ are equal. Recall from $\$ 4$ that $\overline{\mathbf{B}}_{6}$ can be embedded in $\prod_{\mathcal{Q}_{6}^{\prime}} \mathbb{P}^{2}$, where $\mathcal{Q}_{6}^{\prime}$ is the set of ordered quintuples $v=\left(v_{1}, \ldots, v_{5}\right)$ of distinct indices in $\{1, \ldots, 6\}$ such that $v_{1}<\ldots<v_{4}$. We claim that for all 30 quintuples $v \in \mathcal{Q}_{6}^{\prime}$,

$$
\lim _{t \rightarrow 0} \psi_{a_{v_{1}}(t), \ldots, a_{v_{4}}(t)}\left(a_{v_{5}}(t)\right)=\lim _{t \rightarrow 0} \psi_{b_{v_{1}}(t), \ldots, b_{v_{4}}(t)}\left(b_{v_{5}}(t)\right),
$$

where the morphism $\psi$ is defined in $\$ 4$. The limits are computed in Table 1 .

Next we compute $\mathbb{P}\left(\Sigma_{\mathbf{a}}\right)_{\mathbb{k}}$ and $\mathbb{P}\left(\Sigma_{\mathbf{b}}\right)_{\mathbb{k}}$. The respective stable lattices are given by

$$
\begin{aligned}
& \Sigma_{\mathbf{a}}=\left\{\left[e_{1} R+e_{2} R+e_{3} R\right],\left[t e_{1} R+e_{2} R+t^{2} e_{3} R\right],\left[e_{1} R+t e_{2} R+t^{2} e_{3} R\right],\right. {\left.\left[e_{1} R+e_{2} R+t^{2} e_{3} R\right],\left[e_{1} R+t e_{2} R+t e_{3} R\right],\left[t e_{1} R+e_{2} R+t e_{3} R\right]\right\}, } \\
& \Sigma_{\mathbf{b}}=\left\{\left[e_{1} R+e_{2} R+e_{3} R\right],\left[t e_{1} R+e_{2} R+t^{3} e_{3} R\right],\left[e_{1} R+t^{2} e_{2} R+t^{3} e_{3} R\right],\right. \\
& {\left.\left[t e_{1} R+e_{2} R+t e_{3} R\right],\left[e_{1} R+t^{2} e_{2} R+t^{2} e_{3} R\right],\left[e_{1} R+e_{2} R+t^{3} e_{3} R\right]\right\} . }
\end{aligned}
$$

We compute the central fibers $\mathbb{P}\left(\Sigma_{\mathbf{a}}\right)_{\mathbb{k}} \neq \mathbb{P}\left(\Sigma_{\mathbf{b}}\right)_{\mathbb{k}}$ using Remark 5.3 (see Figure 5). The surfaces are illustrated in Figure 6, where $\mathbb{P}\left(\Sigma_{\mathbf{a}}\right)_{\mathbb{k}}$ is on the left and $\mathbb{P}\left(\Sigma_{\mathbf{b}}\right)_{\mathbb{k}}$ is on the right.

TABle 1. Coordinates of the limit $\overline{\mathbf{a}}(0)=\overline{\mathbf{b}}(0) \in \overline{\mathbf{B}}_{6} \subseteq \prod_{Q_{6}^{\prime}} \mathbb{P}^{2}$.

\begin{tabular}{c|c||c|c}
\hline Quintuple & Limit in $\mathbb{P}^{2}$ & Quintuple & Limit in $\mathbb{P}^{2}$ \\
\hline 12345 & {$[1: 0: 0]$} & 13465 & {$[1: 0: 0]$} \\
12346 & {$[0: 1: 0]$} & 13562 & {$[1: 1: 1]$} \\
12354 & {$[0: 0: 1]$} & 13564 & {$[0: 1: 0]$} \\
12356 & {$[0: 1: 0]$} & 14562 & {$[1: 1: 1]$} \\
12364 & {$[0: 0: 1]$} & 14563 & {$[0: 1: 0]$} \\
12365 & {$[1: 0: 0]$} & 23451 & {$[1: 1: 1]$} \\
12453 & {$[0: 0: 1]$} & 23456 & {$[1: 0: 0]$} \\
12456 & {$[0: 1: 0]$} & 23461 & {$[0: 1: 1]$} \\
12463 & {$[0: 0: 1]$} & 23465 & {$[0: 1: 1]$} \\
12465 & {$[1: 0: 0]$} & 23561 & {$[0: 0: 1]$} \\
12563 & {$[1: 0: 1]$} & 23564 & {$[0: 1: 0]$} \\
12564 & {$[1: 0: 1]$} & 24561 & {$[0: 0: 1]$} \\
13452 & {$[0: 1: 1]$} & 24563 & {$[0: 1: 0]$} \\
13456 & {$[0: 1: 1]$} & 34561 & {$[0: 0: 1]$} \\
13462 & {$[1: 1: 1]$} & 34562 & {$[1: 1: 1]$} \\
\hline
\end{tabular}



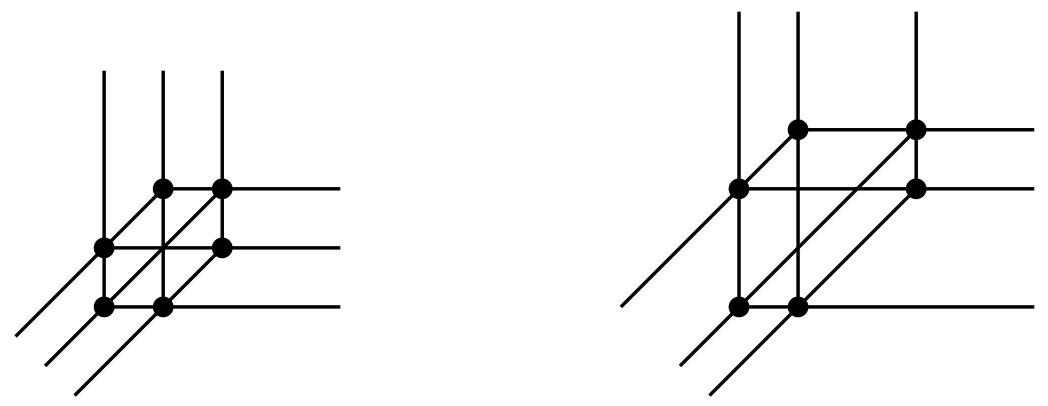

Figure 5. The lattice classes in $\Sigma_{\mathbf{a}}$ and $\Sigma_{\mathbf{b}}$ in the standard apartment.
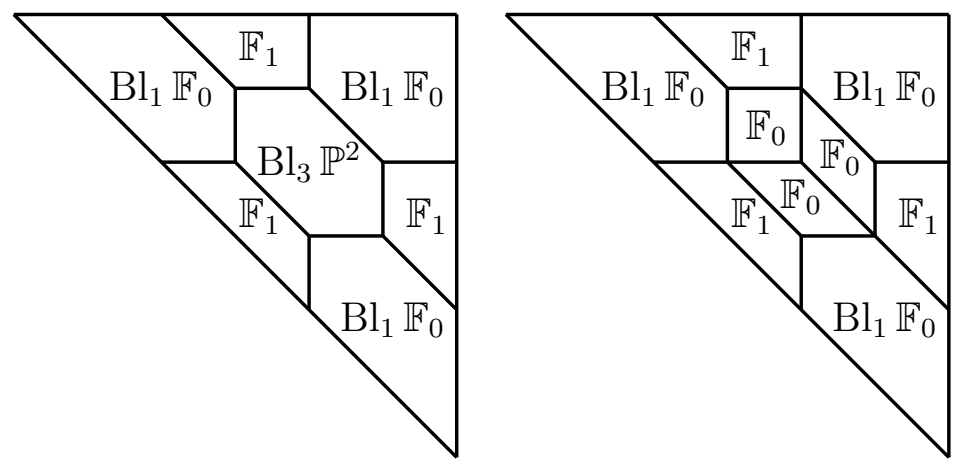

Figure 6 . Example of $\mathbb{P}\left(\Sigma_{\mathbf{a}}\right)_{\mathbb{k}}$ and $\mathbb{P}\left(\Sigma_{\mathbf{b}}\right)_{\mathbb{k}}$ with $\overline{\mathbf{a}}(0)=\overline{\mathbf{b}}(0) \in \overline{\mathbf{B}}_{6}$.

Let us also pinpoint a mistake in the proof of [GP91, §4, Proposition 1] and then explain how to construct the correct moduli space of Mustafin joins. Consider the diagram

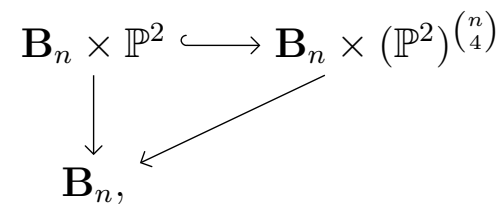

where the horizontal map $\Psi$ sends

$$
\left(\left(p_{1}, \ldots, p_{n}\right), p\right) \mapsto\left(\left(p_{1}, \ldots, p_{n}\right),\left(\psi_{p_{v_{1}}, \ldots, p_{v_{4}}}(p)\right)_{v}\right)
$$

(Recall from $\$ 4$ that, for any quadruple $v_{1}, \ldots, v_{4}, \psi_{p_{v_{1}}, \ldots, p_{v_{4}}}$ is the unique element of $\mathrm{PGL}_{3}$ sending $p_{v_{1}}, \ldots, p_{v_{4}}$ to the standard frame.) Let $\overline{\mathbf{F}}_{n}$ be the closure of the image of $\Psi$ in $\overline{\mathbf{B}}_{n} \times$ $\left(\mathbb{P}^{2}\right)^{\left(\begin{array}{c}n \\ 4\end{array}\right)}$ and consider a morphism $\mu: \overline{\mathbf{F}}_{n} \rightarrow \overline{\mathbf{B}}_{n}$. It was assumed in GP91, §4, Proposition 1] that formation of $\mu$ commutes with arbitrary base-changes $S \rightarrow \mathbf{B}_{n}$. However, this is wrong (Example 7.1), in particular $\mu$ is not flat. A remedy is provided by the Grothendieck's universal flattening morphism as in Ray72. Consider the morphism

$$
\Phi: \mathbf{B}_{n} \rightarrow \overline{\mathbf{B}}_{n} \times \operatorname{Hilb}\left(\left(\mathbb{P}^{2}\right)\left(\begin{array}{c}
n \\
4
\end{array}\right)\right)
$$


where the first component is the inclusion and the second component sends $\left(p_{1}, \ldots, p_{n}\right) \in \mathbf{B}_{n}$ to $\Psi\left(\left(p_{1}, \ldots, p_{n}\right) \times \mathbb{P}^{2}\right)$. By Hilb $\left(\left(\mathbb{P}^{2}\right)^{\left(\begin{array}{c}n \\ 4\end{array}\right)}\right)$ we mean the connected component of the Hilbert scheme of closed subschemes in $\left(\mathbb{P}^{2}\right)^{\left(\begin{array}{l}n \\ 4\end{array}\right)}$ which parametrizes the diagonally embedded $\mathbb{P}^{2}$ in $\left(\mathbb{P}^{2}\right)^{\left(\begin{array}{c}n \\ 4\end{array}\right)}$. The correct moduli space is the closure of the image of $\Phi$ and the universal Mustafin join for arcs is the pullback of the universal family of the Hilbert scheme. We will analyze this construction in detail, although we will use the multigraded Hilbert scheme of $\left(\mathbb{P}^{2}\right)^{\left(\begin{array}{c}n \\ 4\end{array}\right)}$ instead of the usual Hilbert scheme in order to have Proposition 7.10.

Definition 7.2 ([HS04]). For a commutative ring $k$, let $S=k\left[x_{1}, \ldots, x_{n}\right]$ be a polynomial ring with a grading by an abelian group $A$ given by a semigroup homomorphism deg: $\mathbb{N}^{n} \rightarrow$ $A$. Fix a function

$$
h: A \rightarrow \mathbb{N} .
$$

The multigraded Hilbert scheme $\mathbf{H}_{S}^{h}$ parametrizes all ideals in $S$ homogeneous with respect to deg and with Hilbert function $h$, i.e. such that

$$
\operatorname{dim}_{k} S_{a} / I_{a}=h(a) \text { for every } a \in A \text {. }
$$

For every $k$-algebra $R$, the set of $R$-points $\mathbf{H}_{S}^{h}(R)$ is the set of homogeneous ideals $I \subseteq R \otimes_{k} S$ such that $(R \otimes S)_{a} / I_{a}$ is a locally free $R$-module of rank $h(a)$ for every $a$. The scheme $\mathbf{H}_{S}^{h}$ is quasi-projective over $k$, in fact projective if $1 \in S$ is the only monomial of degree 0 .

Example 7.3. We are interested in the multigraded Hilbert scheme of $\left(\mathbb{P}^{2}\right)^{N}$. Here

$$
S=\mathbb{k}\left[x_{i j} \mid i=1,2,3, j=1, \ldots, N\right],
$$

where $\mathbb{k}$ is our algebraically closed base field and $\left[x_{1 j}: x_{2 j}: x_{3 j}\right]$ are homogeneous coordinates of the $j$-th copy of $\mathbb{P}^{2}$, with the usual multigrading by $\mathbb{Z}^{N}$. The numerical function $h$ is the Hilbert function of the diagonally embedded $\mathbb{P}^{2} \hookrightarrow\left(\mathbb{P}^{2}\right)^{N}$. A detailed study of this case can be found in [CS10]. The authors prove that $\mathbf{H}_{S}^{h}$ is connected and all ideals parametrized by it are radical and Cohen-Macaulay. The morphism from $\mathbf{H}_{S}^{h}$ to the Hilbert scheme of $\left(\mathbb{P}^{2}\right)^{N}$ given by taking $\operatorname{Proj}\left(R \otimes_{\mathbb{k}} S\right) / I$ is injective on $\mathbb{k}$-points, although it is not clear if it is a closed embedding. It follows that

Lemma 7.4. The projection from $\left(\mathbb{P}^{2}\right)^{N}$ to $\left(\mathbb{P}^{2}\right)^{N-1}$ induces a morphism of multigraded Hilbert schemes (with the Hilbert function of the diagonal).

Proof. Let $S$ and $S^{\prime}$ be multigraded coordinate rings of $\left(\mathbb{P}^{2}\right)^{N}$ and $\left(\mathbb{P}^{2}\right)^{N-1}$. The natural transformation of functors of points takes a multihomogeneous ideal $I \subseteq R \otimes_{\mathbb{k}} S$ to the ideal of the projection, which is $I \cap\left(R \otimes_{\mathbb{k}} S^{\prime}\right)$. Its numerical function is $h^{\prime}=h\left(a_{1}, \ldots, a_{N-1}, 0\right)$.

Definition 7.5. Let $\mathbf{H}_{S}^{h}$ be the multigraded Hilbert scheme of $\left(\mathbb{P}^{2}\right)^{\left(\begin{array}{c}n \\ 4\end{array}\right)}$ as in Example 7.3 (with $N=\left(\begin{array}{l}n \\ 4\end{array}\right)$ ). The diagram (5) induces an embedding

$$
\Phi: \mathbf{B}_{n} \hookrightarrow \overline{\mathbf{B}}_{n} \times \mathbf{H}_{S}^{h} .
$$

Let $\overline{\mathbf{X}}_{\mathrm{GP}}(3, n)$ be the Zariski closure of the image of $\Phi$. Let $\overline{\mathcal{M}} \rightarrow \overline{\mathbf{X}}_{\mathrm{GP}}(3, n)$ be the pullback of the universal family of the (usual, not multigraded) Hilbert scheme. 
Theorem 7.6. The family $\overline{\mathcal{M}}$ is the universal Mustafin join for point configurations. Concretely, take an arc $\mathbf{a}: \operatorname{Spec}(K) \rightarrow \mathbf{B}_{n}$ and its unique extension $\overline{\mathbf{a}}: \operatorname{Spec}(R) \rightarrow \overline{\mathbf{X}}_{\mathrm{GP}}(3, n)$. Then $\overline{\mathbf{a}}^{*} \overline{\mathcal{M}}$ is isomorphic to the Mustafin join $\mathbb{P}\left(\Sigma_{\mathbf{a}}\right)$, where $\Sigma_{\mathbf{a}}$ is the set of stable lattices with respect to $\mathbf{a}$.

Proof. Recall that $\mathbb{P}\left(\Sigma_{\mathbf{a}}\right) \rightarrow \operatorname{Spec}(R)$ is flat and proper, and that $\mathbb{P}\left(\Sigma_{\mathbf{a}}\right)$ is the Zariski closure of $\operatorname{Spec}(K) \times \mathbb{P}^{2}$ inside $\prod_{L \in \Sigma_{\mathbf{a}}} \mathbb{P}(L)$. Therefore, we have the following commutative diagram:

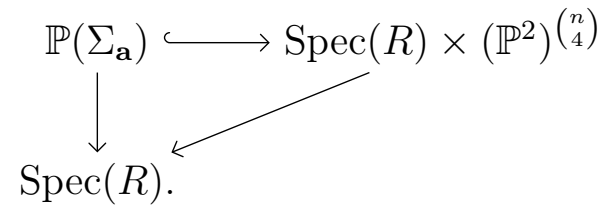

The horizontal map comes from the fact that for each quadruple, there is a unique stable lattice $L \in \Sigma_{\mathbf{a}}$ stabilizing it. The diagram induces a morphism from $\operatorname{Spec}(R)$ to the (usual)

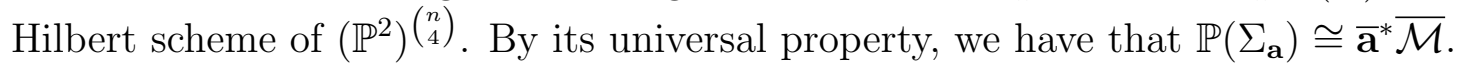

Remark 7.7. The corresponding $R$-point of $\mathbf{H}_{S}^{h}$, i.e. the multi-homogeneous ideal of $\mathbb{P}\left(\Sigma_{\mathbf{a}}\right)$, can be computed as in Remark 5.1 .

Corollary 7.8. We have a birational morphism $\overline{\mathbf{X}}_{\mathrm{GP}}(3, n) \rightarrow \overline{\mathbf{B}}_{n}$. In particular, we have a morphism of normalizations $\overline{\mathbf{X}}_{\mathrm{GP}}(3, n)^{\nu} \rightarrow \overline{\mathbf{B}}_{n}^{\nu} \cong \overline{\mathbf{X}}(3, n)^{\nu}$.

Proof. This follows from Definition 7.5. As $\overline{\mathbf{X}}_{\mathrm{GP}}(3, n) \subseteq \overline{\mathbf{B}}_{n} \times \mathbf{H}_{S}^{h}$, we have a morphism $\overline{\mathbf{X}}_{\mathrm{GP}}(3, n) \rightarrow \overline{\mathbf{B}}_{n}$ given by the restriction of the projection onto the first factor. This morphism is birational as it restricts to the identity on $\mathbf{B}_{n}$.

Theorem 7.9. We have an isomorphism $\overline{\mathrm{X}}_{\mathrm{GP}}(3,5) \cong \overline{\mathbf{B}}_{5} \cong \overline{\mathrm{M}}_{0,5}$.

Proof. Since $\overline{\mathbf{B}}_{5} \cong \overline{\mathrm{M}}_{0,5}$ is normal (in fact smooth), it suffices to exhibit a family of Mustafin joins for point configurations over $\overline{\mathrm{M}}_{0,5}$ inducing a morphism to Hilb $\left(\left(\mathbb{P}^{2}\right)^{\left(\begin{array}{l}5 \\ 4\end{array}\right)}\right)$.

The space $\overline{\mathrm{M}}_{0,5}$ is isomorphic to Hassett's moduli space $\overline{\mathrm{M}}_{0,\left(\frac{1}{2}\right)^{5}}$ [Has03]. This gives a smooth conic bundle $\pi: \overline{\mathcal{C}} \rightarrow \overline{\mathrm{M}}_{0,5}$ with sections $s_{1}, \ldots, s_{5}$ such that at most two sections are equal on each fiber. The relative anti-canonical divisor $-K_{\pi}$ induces an embedding of $\overline{\mathcal{C}}$ into the $\mathbb{P}^{2}$-bundle $\overline{\mathcal{P}}^{\prime \prime \prime}$ with an associated vector bundle the pushforward of $-K_{\pi}$. So we have a $\mathbb{P}^{2}$-bundle $\overline{\mathcal{P}}^{\prime \prime \prime} \rightarrow \overline{\mathrm{M}}_{0,5}$ with 5 sections. Its fibers over a general point of a 1 -stratum (resp. over a 0 -stratum) of $\overline{\mathrm{M}}_{0,5}$ are illustrated in the left picture of Figure 7 (resp. the left picture of Figure 8).

For distinct $i, j \in\{1, \ldots, 5\}$, the curves $C_{i j}=\operatorname{im}\left(s_{i}\right) \cap \operatorname{im}\left(s_{j}\right)$ are smooth and pairwise disjoint. Let $\overline{\mathcal{P}}^{\prime \prime}$ be the blow up of $\overline{\mathcal{P}}^{\prime \prime \prime}$ along these curves. The fibers of $\overline{\mathcal{P}}^{\prime \prime} \rightarrow \overline{\mathrm{M}}_{0,5}$ over a general point of a 1-stratum (resp. over a 0 -stratum) of $\overline{\mathrm{M}}_{0,5}$ are illustrated in the right picture of Figure 7 (resp. the second picture from the left in Figure 8).

Given distinct $i, j, k, \ell \in\{1, \ldots, 5\}$, let $p \in \overline{\mathrm{M}}_{0,5}$ be the 0 -stratum such that $s_{i}(p)=$ $s_{j}(p), s_{k}(p)=s_{\ell}(p)$, and let $L_{i j, k \ell} \subseteq \overline{\mathcal{P}}^{\prime \prime \prime}$ be the line in the $\mathbb{P}^{2}$ over $p$ passing through these 

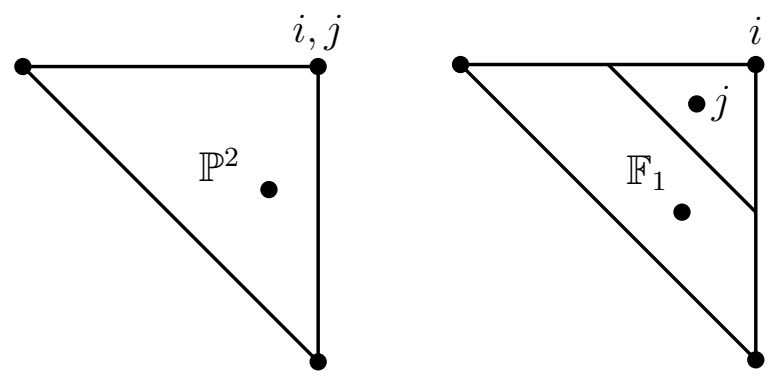

FiguRE 7. Fibers of families over a general point of the 1-strata.
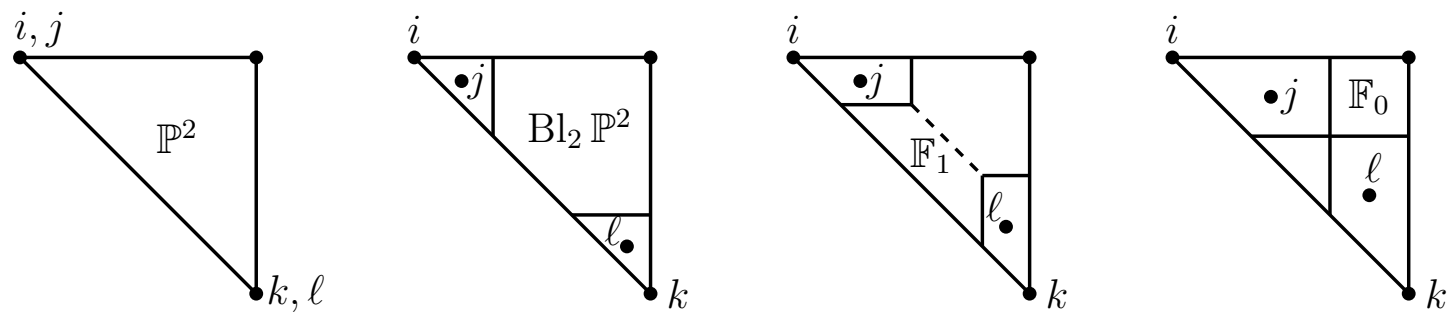

FIgURE 8. Fibers of families over the 0 -strata.

two points. Let $\overline{\mathcal{P}}^{\prime}$ be the blow up of $\overline{\mathcal{P}}^{\prime \prime}$ along the strict transforms of the lines $L_{i j, k \ell}$. This blow up affects the fibers over the 0 -strata, and the fiber $X$ of $\overline{\mathcal{P}}^{\prime} \rightarrow \overline{\mathrm{M}}_{0,5}$ over a 0 -stratum is illustrated in the third picture from the left in Figure 8. The (-1)-curve $E \subseteq X$ on the $\mathbb{F}_{1}$ component is also a (-1)-curve in $\mathrm{Bl}_{2} \mathbb{P}^{2}$ (this is the curve dashed in Figure 8). We claim that $E$ can be contracted. Up to relabeling, we can assume $s_{1}(p)=s_{2}(p)$ and $s_{3}(p)=s_{4}(p)$. Endow the family $\mathbb{P}_{\mathrm{M}_{0,5}}^{2} \rightarrow \mathrm{M}_{0,5}$ with the relative effective Cartier divisor $\mathcal{D} \subseteq \mathbb{P}_{\mathrm{M}_{0,5}}$ giving on the general fiber the line arrangement shown on the left of Figure 9. The Zariski closure $\overline{\mathcal{D}} \subseteq \overline{\mathcal{P}}^{\prime}$ specializes to $X$ giving the line arrangement $D_{p}$ shown on the right of Figure 9 .
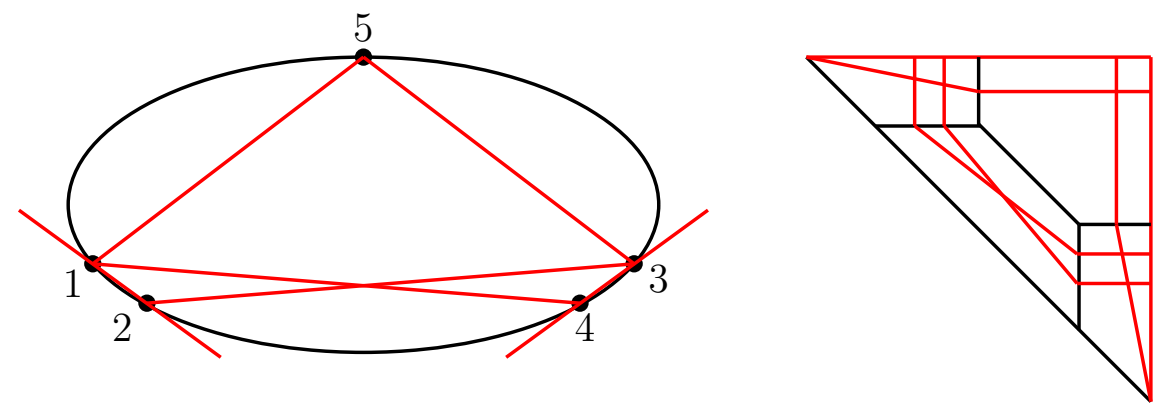

Figure 9. In red, a fiber of $\overline{\mathcal{D}}$ over a point in $\mathrm{M}_{0,5}$ (left) and a 0 -stratum (right). 
The pair $\left(X, D_{p}\right)$ is semi-log canonical and $K_{X}+D_{p}$ is nef but not ample: its intersection with $E$ is 0 . Applying the relative minimal model program to $\left(\overline{\mathcal{P}}^{\prime}, K+\overline{\mathcal{D}}\right) \rightarrow \overline{\mathrm{M}}_{0,5}$ in the neighborhood of $p$ gives a morphism contracting exactly $E$ over an open neighborhood of $p$. Similarly, we can construct the contractions of the remaining 14 curves of type $E$ and glue them to obtain a small contraction $\overline{\mathcal{P}}^{\prime} \rightarrow \overline{\mathcal{P}}$. A priori, $\overline{\mathcal{P}}$ is an algebraic space, but, as we are going to see later, it is actually a projective variety. The fibers of $\gamma: \overline{\mathcal{P}} \rightarrow \overline{\mathrm{M}}_{0,5}$ over the 0 strata are described by the rightmost picture in Figure 8 . We denote by $\widetilde{s}_{1}, \ldots, \widetilde{s}_{5}: \overline{\mathrm{M}}_{0,5} \rightarrow \overline{\mathcal{P}}$ the strict transforms of the sections $s_{1}, \ldots, s_{5}$.

To define a morphism $\overline{\mathrm{M}}_{0,5} \rightarrow \overline{\mathbf{X}}_{\mathrm{GP}}(3,5)$, we first construct a commutative diagram

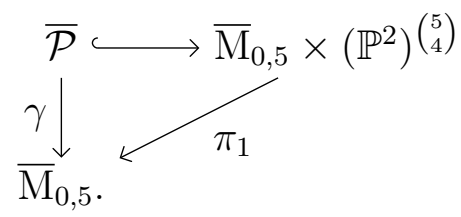

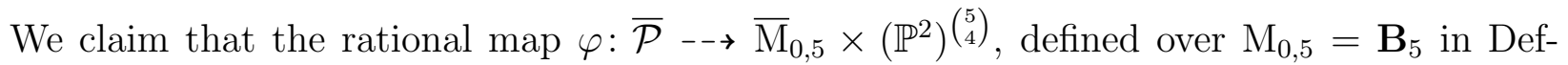
inition 7.5, is regular. In other words, each of the $\left(\begin{array}{l}5 \\ 4\end{array}\right)$ maps from $\overline{\mathcal{P}}$ to $\mathbb{P}^{2}$ is regular. Let $C \subseteq \overrightarrow{\mathrm{M}}_{0,5}$ be the open part of a 1-stratum, e.g. the one corresponding to the degeneration where $s_{1}(C)=s_{2}(C)$. The fibers of $\gamma$ over $C$ are illustrated at the top of Figure 10 .

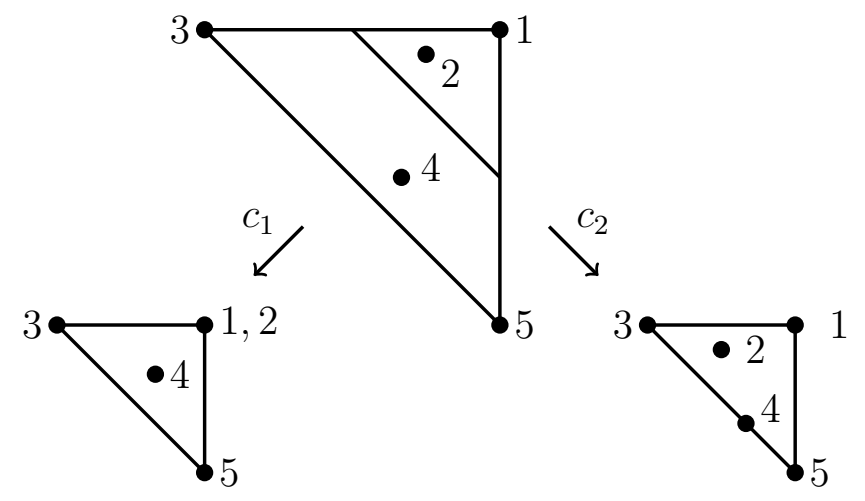

Figure 10. Effect of the contractions $c_{1}, c_{2}$ on the fiber $\gamma^{-1}(p)$ for $p$ in the interior of a 1-stratum of $\overline{\mathrm{M}}_{0,5}$.

Let $U=\mathrm{M}_{0,5} \cup C$ be an open neighborhood of $C$ in $\overline{\mathrm{M}}_{0,5}$. We have contractions $c_{1}$ and $c_{2}$ defined on $\gamma^{-1}(U)$ such that, over $C, c_{1}$ fiberwisely collapses the $\mathbb{P}^{2}$ component to a point, and $c_{2}$ contracts $\mathbb{F}_{1}$ along the ruling. The effect of these contractions on the fibers over $C$ is represented in Figure 10. These contractions are constructed by applying the relative $K+D$ minimal model program for an appropriate divisor $D$ on $\gamma^{-1}(U)$. Namely, on the fiber $\mathbb{P}^{2}$ over a point in $\mathrm{M}_{0,5}$, we consider the lines $\overline{13}, \overline{14}, \overline{35}, \overline{45}$ (resp. $\overline{12}, \overline{13}, \overline{14}, \overline{15})$, and the divisor $D$ is their closure in $\gamma^{-1}(U)$. The induced contraction is $c_{1}$ (resp. $c_{2}$ ). We define $\bar{\varphi}_{U}: \gamma^{-1}(U) \rightarrow U \times\left(\mathbb{P}^{2}\right)^{\left(\begin{array}{l}5 \\ 4\end{array}\right)}$ as follows. Given $x \in \gamma^{-1}(U)$, let 
$\bar{\varphi}_{U}(x)=\left(\gamma(x),\left(q_{i}\right)_{i=1}^{5}\right)$, where the $q_{i}$ are defined as follows. For exactly one $j \in\{1,2\}$, the four points $c_{j}\left(\widetilde{s}_{1}(x)\right), \ldots, c_{j}\left(\widetilde{s_{i}(x)}\right), \ldots, c_{j}\left(\widetilde{s}_{5}(x)\right) \in \mathbb{P}^{2}$ are in general linear position. So define $q_{i}$ to be the image of $c_{j}(x)$ under the unique projective linear transformation sending $\left.c_{j}\left(\widetilde{s}_{1}(x)\right), \ldots, \widehat{c_{j}\left(\widetilde{s}_{i}(x)\right.}\right), \ldots, c_{j}\left(\widetilde{s}_{5}(x)\right) \in \mathbb{P}^{2}$ in standard position.

Let $p$ be a 0 -stratum of $\overline{\mathrm{M}}_{0,5}$, for example suppose it corresponds to a degeneration where $s_{1}(p)=s_{2}(p)$ and $s_{3}(p)=s_{4}(p)$. The fiber $\gamma^{-1}(p)$ is in the top right corner of Figure 11. The

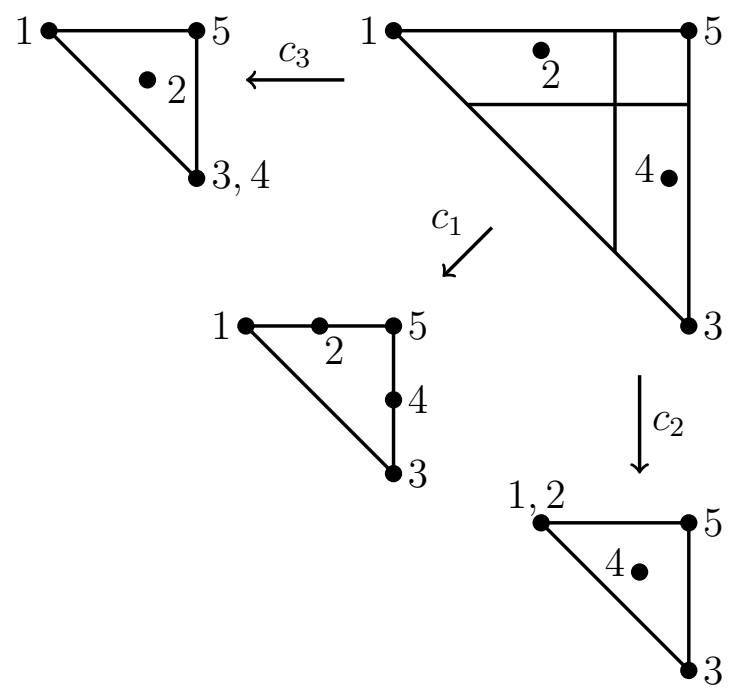

Figure 11. Contractions $c_{1}, c_{2}, c_{3}$ on the fiber $\gamma^{-1}(p)$.

points $\widetilde{s}_{1}(p), \widetilde{s}_{2}(p)$ are contained in one copy of $\mathbb{F}_{1}, \widetilde{s}_{3}(p), \widetilde{s}_{4}(p)$ are contained in the other copy of $\mathbb{F}_{1}$, and $\widetilde{s}_{5}(p) \in \mathbb{F}_{0}$. Let $U \subseteq \overline{\mathrm{M}}_{0,5}$ be an open neighborhood of $p$ avoiding all the strata of $\overline{\mathrm{M}}_{0,5}$ not specializing to $p$. Using the relative minimal model program as above, we can construct three contractions of $\gamma^{-1}(U)$ which we label $c_{1}$ (use the lines $\overline{14}, \overline{15}, \overline{23}, \overline{35}$ ), $c_{2}$ (use the lines $\overline{14}, \overline{15}, \overline{34}, \overline{35}$ ), and $c_{3}$ (use the lines $\overline{12}, \overline{15}, \overline{23}, \overline{35}$ ). See Figure 11 for the effect of these contractions on the fiber over $p$. The fibers over the adjacent 1-strata are contracted accordingly. We define $\bar{\varphi}_{U}(x)=\left(\gamma(x),\left(q_{i}\right)_{i=1}^{5}\right)$ as in the previous case.

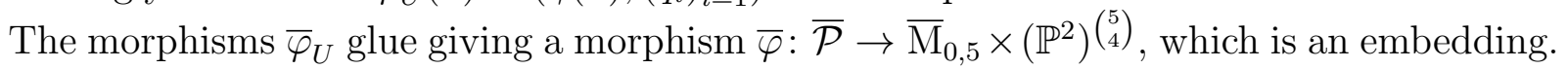
Indeed, by Nakayama's lemma, it suffices to show that the restriction to each fiber is a closed

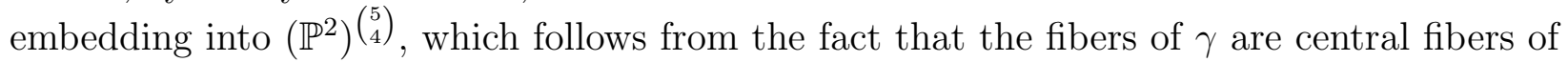
Mustafin joins (see Remark 5.5). Let $\overline{\mathbf{X}}_{\mathrm{GP}}^{\prime}(3,5)$ be the Zariski closure of the image of $\mathbf{B}_{5}$ in $\overline{\mathbf{B}}_{5} \times \operatorname{Hilb}\left(\left(\mathbb{P}^{2}\right)^{\left(\begin{array}{l}5 \\ 4\end{array}\right)}\right)$. The morphism $\bar{\varphi}$ induces a birational morphism $\overline{\mathrm{M}}_{0,5} \rightarrow \overline{\mathbf{X}}_{\mathrm{GP}}^{\prime}(3,5)$. Since we also have a birational morphism $\overline{\mathbf{X}}_{\mathrm{GP}}^{\prime}(3,5) \rightarrow \overline{\mathbf{B}}_{5} \cong \overline{\mathrm{M}}_{0,5}$, we have $\overline{\mathbf{X}}_{\mathrm{GP}}^{\prime}(3,5) \cong \overline{\mathrm{M}}_{0,5}$. By [CS10, Proposition 3.1], the morphism $\overline{\mathbf{X}}_{\mathrm{GP}}(3,5) \rightarrow \overline{\mathbf{X}}_{\mathrm{GP}}^{\prime}(3,5)$ is bijective. Since $\overline{\mathrm{M}}_{0,5}$ is normal, $\overline{\mathrm{X}}_{\mathrm{GP}}(3,5) \cong \overline{\mathrm{X}}_{\mathrm{GP}}^{\prime}(3,5) \cong \overline{\mathrm{M}}_{0,5}$ by Zariski's Main Theorem. 
Proposition 7.10. For each $i=1, \ldots, n$, there exists a forgetful morphism

$$
\overline{\mathbf{X}}_{\mathrm{GP}}(3, n) \rightarrow \overline{\mathbf{X}}_{\mathrm{GP}}(3, n-1)
$$

extending the obvious forgetful map $\mathbf{B}_{n} \rightarrow \mathbf{B}_{n-1}$ that forgets the $i$-th point.

Proof. Say $i=n$. Following the definition of $\overline{\mathbf{X}}_{\mathrm{GP}}(3, n)$ as the closure of $\mathbf{B}_{n}$ in the product of $\overline{\mathbf{B}}_{n}$ and the multigraded Hilbert scheme and the definition of $\overline{\mathbf{B}}_{n}$ as the closure of $\mathbf{B}_{n}$ in $\left(\mathbb{P}^{1}\right)^{\mathcal{Q}_{n}}$, we need to show two things. Firstly, the projection from $\left(\mathbb{P}^{1}\right)^{\mathcal{Q}_{n}}$ to $\left(\mathbb{P}^{1}\right)^{\mathcal{Q}_{n-1}}$ induces the forgetful morphism $\overline{\mathbf{B}}_{n} \rightarrow \overline{\mathbf{B}}_{n-1}$, which is clear. Secondly, the projection from $\left(\mathbb{P}^{2}\right)^{\left(\begin{array}{l}n \\ 4\end{array}\right)}$ to $\left(\mathbb{P}^{2}\right)^{\left(\begin{array}{c}n-1 \\ 4\end{array}\right)}$ induces a morphism of multigraded Hilbert schemes (with the Hilbert function of the diagonal), which is Lemma 7.4.

Definition 7.11. Let $I \in\left(\begin{array}{c}{[n]} \\ 5\end{array}\right), i \in I$. Consider the composition of forgetful morphisms

$$
\overline{\mathbf{X}}_{\mathrm{GP}}(3, n) \rightarrow \overline{\mathrm{X}}_{\mathrm{GP}}(3, I) \cong \overline{\mathrm{M}}_{0,5},
$$

and denote by $f_{I, i}$ the composition of this map with the $i$-th Kapranov's map $\overline{\mathrm{M}}_{0,5} \rightarrow \mathbb{P}^{2}$.

Given $k \in\{1, \ldots, n\}$, let $\left(\begin{array}{c}{[n]} \\ 4\end{array}\right)_{k} \subseteq\left(\begin{array}{c}{[n]} \\ 4\end{array}\right)$ be the subset of quadruples $J=\left\{j_{1}<\ldots<j_{4}\right\}$ containing $k$. Then define $\chi_{k}:\left(\begin{array}{c}{[n]} \\ 4\end{array}\right)_{k} \rightarrow \mathbb{P}^{2}$ to be the following function:

$$
\chi_{k}(J)=\left\{\begin{array}{l}
{[1: 0: 0] \text { if } k=j_{1}} \\
{[0: 1: 0] \text { if } k=j_{2}} \\
{[0: 0: 1] \text { if } k=j_{3}} \\
{[1: 1: 1] \text { if } k=j_{4} .}
\end{array}\right.
$$

Finally, for $k=1, \ldots, n$, consider the morphism

$$
s_{k}: \overline{\mathbf{X}}_{\mathrm{GP}}(3, n) \rightarrow \overline{\mathbf{X}}_{\mathrm{GP}}(3, n) \times\left(\mathbb{P}^{2}\right)^{\left(\begin{array}{c}
n \\
4
\end{array}\right)}
$$

defined as

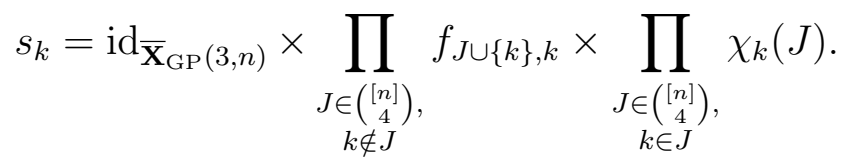

Theorem 7.12. The morphisms $s_{1}, \ldots, s_{n}$ are sections $\overline{\mathbf{X}}_{\mathrm{GP}}(3, n) \rightarrow \overline{\mathcal{M}}$ of the universal Mustafin join of point configurations. They give $n$ smooth distinct points on each fiber.

Proof. Since we already know that $s_{k}$ maps the interior of $\overline{\mathbf{X}}_{\mathrm{GP}}(3, n)$ into $\overline{\mathcal{M}}$, the image of $s_{k}$ is contained in $\overline{\mathcal{M}}$ by continuity. Let $x \in \overline{\mathbf{X}}_{\mathrm{GP}}(3, n)$. Let $\mathbf{a}: \operatorname{Spec}(K) \rightarrow \mathbf{B}_{n}$ be an arc such that the unique extension $\overline{\mathbf{a}}: \operatorname{Spec}(R) \rightarrow \overline{\mathbf{X}}_{\mathrm{GP}}(3, n)$ satisfies $\overline{\mathbf{a}}(0)=x$. Then $\overline{\mathcal{M}} \rightarrow \overline{\mathbf{X}}_{\mathrm{GP}}(3, n)$ and $s_{1}, \ldots, s_{k}$ pullback to $\operatorname{Spec}(R)$ giving the Mustafin join $\mathbb{P}\left(\Sigma_{\mathbf{a}}\right) \rightarrow \operatorname{Spec}(R)$ with the sections $\bar{a}_{1}, \ldots, \bar{a}_{n}$. The points $\bar{a}_{1}(0), \ldots, \bar{a}_{n}(0) \in \mathbb{P}\left(\Sigma_{\mathbf{a}}\right)_{\mathbb{k}}$ are distinct by Lemma 6.1 and smooth by Theorem 6.9, implying the theorem.

Finally, we give a criterion to establish which $n$-pointed degenerations of $\mathbb{P}^{2}$ arise as fibers of the universal Mustafin join $\overline{\mathcal{M}} \rightarrow \overline{\mathbf{X}}_{\mathrm{GP}}(3, n)$ of point configurations.

Lemma 7.13. Let $X$ be a projective surface with smooth points $p_{1}, \ldots, p_{n} \in X$ such that 
(1) There exists $\Sigma=\left\{\left[L_{1}\right], \ldots,\left[L_{m}\right]\right\} \subseteq \mathfrak{B}_{3}^{0}$ such that $X \cong \mathbb{P}(\Sigma)_{\mathbb{k}}$ (recall that if $\Sigma$ is contained in an apartment, then $\mathbb{P}(\Sigma)_{\mathbb{k}}$ can be easily computed using Remark 5.3);

(2) There exists a surjective map $\left(\begin{array}{c}{[n]} \\ 4\end{array}\right) \rightarrow \Sigma$ associating to a quadruple of distinct indices $i, j, k, h \in[n]$ a lattice class $\left[L_{r}\right] \in \Sigma$ such that the images of the points $p_{i}, p_{j}, p_{k}, p_{h}$ under the composition $X \cong \mathbb{P}(\Sigma)_{\mathbb{k}} \rightarrow \mathbb{P}\left(L_{r}\right)_{\mathbb{k}}$ are in general linear position.

Then there exists $\mathbf{a} \in\left(\mathbb{P}^{2}\right)^{n}(K)$ in general linear position such that $X \cong \mathbb{P}\left(\Sigma_{\mathbf{a}}\right)_{\mathbb{k}}$, and $p_{1}, \ldots, p_{n}$ correspond respectively to $\bar{a}_{1}(0), \ldots, \bar{a}_{n}(0)$ under this isomorphism.

Proof. Since $p_{1}, \ldots, p_{n}$ are smooth points of $X$ (and hence smooth points of $\left.\mathbb{P}(\Sigma)_{\mathbb{k}}\right)$, by the infinitesimal lifting property we can find $\mathbf{a} \in\left(\mathbb{P}^{2}\right)^{n}(K)$ in general linear position such that $\bar{a}_{1}(0), \ldots, \bar{a}_{n}(0) \in \mathbb{P}(\Sigma)_{\mathbb{k}}$ correspond respectively to $p_{1}, \ldots, p_{n}$ under the isomorphism $\mathbb{P}(\Sigma)_{\mathbb{k}} \cong X$. By the definition of stable lattices and by our assumptions, we have that $\Sigma_{\mathbf{a}}=\Sigma$.

Example 7.14. Let $\left(X ; p_{1}, \ldots, p_{6}\right)$ be the 6 -pointed reducible surface \#8 in Table 14 . We claim that it arises as $\left(\mathbb{P}\left(\Sigma_{\mathbf{a}}\right)_{\mathbb{k}} ; \bar{a}_{1}(0), \ldots, \bar{a}_{6}(0)\right)$ for some arc $\mathbf{a} \in\left(\mathbb{P}^{2}\right)^{6}(K)$ in general linear position. Lemma $7.13(1)$ is satisfied by choosing $\Sigma=\left\{\left[L_{1}\right],\left[L_{2}\right],\left[L_{3}\right]\right\}$, where

$$
L_{1}=e_{1} R+e_{2} R+e_{3} R, L_{2}=t e_{1} R+e_{2} R+t e_{3} R, L_{3}=e_{1} R+t^{2} e_{2} R+t e_{3} R .
$$

Figure 12 shows that the assumption (2) is satisfied as well.

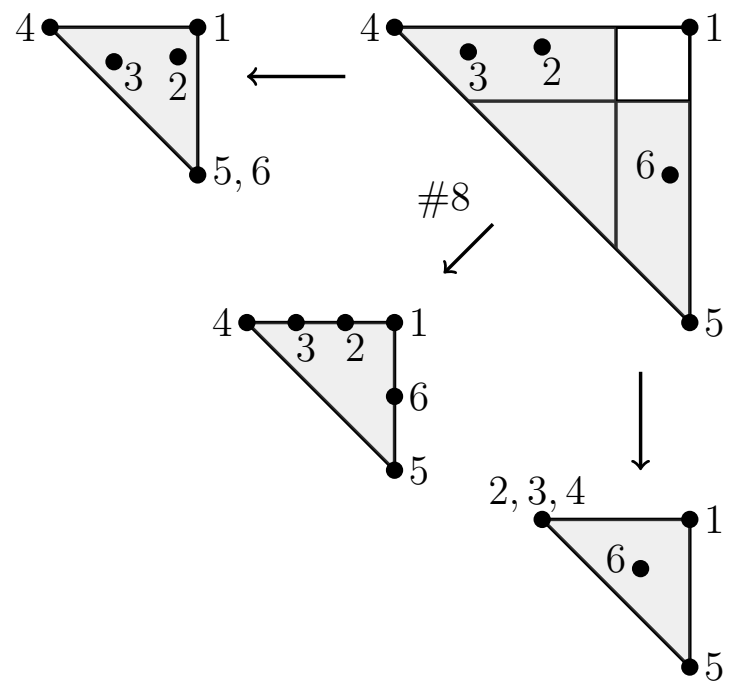

Figure 12. Maps to the primary components in Example 7.14.

\section{Analogue of the Losev-Manin space for moduli of points in $\mathbb{P}^{2}$}

The Grothendieck-Knudsen moduli space $\overline{\mathrm{M}}_{0, n}=\overline{\mathbf{X}}(2, n)$ of stable rational curves has a toric analogue, the Losev-Manin space [LM00, CT20], which parametrizes chains of rational curves with $m$ light and two heavy points (one at each end of the chain). The light points 
can coincide with each other but not with the nodes or the heavy points. The Losev-Manin space is a toric variety associated with the permutohedron. In this and the next sections we will discuss analogues of the Losev-Manin space for moduli spaces of points and lines in $\mathbb{P}^{2}$. We start with a toric analogue of the Kapranov Chow quotient space $\overline{\mathbf{X}}(3, n)=\left(\mathbb{P}^{2}\right)^{n} / / \mathrm{PGL}_{3}$, which will be the Chow quotient $\left(\mathbb{P}^{2}\right)^{m} / / \mathbb{G}_{m}^{2}$ for $n=m+3$.

Denote by $Y_{\mathcal{F}}$ the toric variety associated to a fan $\mathcal{F}$. Let $T \subseteq Y_{\mathcal{F}}$ be the dense open torus. By KSZ91], the Chow quotient $Y_{\mathcal{F}} / / H$ by a subtorus $H \subseteq T$ is a toric variety (not necessarily normal) of the quotient torus $T / H$. The associated fan of the normalization is called the quotient fan. We recall its description in our case: $Y_{\mathcal{F}}=\left(\mathbb{P}^{2}\right)^{m}$ and $H$ is the dense open torus in $\mathbb{P}^{2}$ acting diagonally on $\left(\mathbb{P}^{2}\right)^{m}$. The Chow quotient parametrizes translates $\left(p_{1}^{-1}, \ldots, p_{m}^{-1}\right) \cdot \mathbb{P}^{2} \subseteq\left(\mathbb{P}^{2}\right)^{m}$ of the diagonally embedded $\mathbb{P}^{2} \subseteq\left(\mathbb{P}^{2}\right)^{m}$ and their limits in the Chow variety, where $\left(p_{1}, \ldots, p_{m}\right) \in\left(\mathbb{G}_{m}^{2}\right)^{m}$.

Let $N$ be the lattice of 1 -parameter subgroups of $H$ and let $\mathcal{P}$ be the fan of $\mathbb{P}^{2}$ in $N_{\mathbb{R}} \cong \mathbb{R}^{2}$. The product fan $\mathcal{P}^{m}$ in $N_{\mathbb{R}}^{m}$ gives the toric variety $\left(\mathbb{P}^{2}\right)^{m}$. In what follows, we identify $N_{\mathbb{R}}^{m}$ with $\mathbb{R}^{2 m}$ and we view $\mathbb{R}^{2}$ as diagonally embedded in $\mathbb{R}^{2 m}$. Given $v \in \mathbb{R}^{2 m}$, define

$$
\mathcal{P}_{v}^{m}=\left\{\sigma \in \mathcal{P}^{m} \mid \sigma \cap\left(v+\mathbb{R}^{2}\right) \neq \emptyset\right\} .
$$

Vectors $v, w \in \mathbb{R}^{2 m}$ are called equivalent provided $\mathcal{P}_{v}^{m}=\mathcal{P}_{w}^{m}$. The closures of equivalence classes are rational convex cones in $\mathbb{R}^{2 m}$ invariant under translations by $\mathbb{R}^{2}$. The images of these cones under the quotient map

$$
q: \mathbb{R}^{2 m} \rightarrow \mathbb{R}^{2 m} / \mathbb{R}^{2}
$$

form the fan $\mathcal{Q}_{m}$ called the quotient fan. We call $Y_{\mathcal{Q}_{m}}$ the toric Kapranov space. It is a normalization of the Chow quotient and the (multigraded) Hilbert quotient of $\left(\mathbb{P}^{2}\right)^{m}$ by $H$ (the closure of $T / H$ in the (multigraded) Hilbert scheme of $\left.\left(\mathbb{P}^{2}\right)^{m}\right)$, see Example 7.3 .

We would like to describe cones in $\mathcal{Q}_{m}$ explicitly.

Definition 8.1. Given $\left(v, v^{\prime}\right) \in \mathbb{R}^{2}$, a spider $S\left(v, v^{\prime}\right)$ is the union of three rays in $\mathbb{R}^{2}$ :

$S\left(v, v^{\prime}\right)=\left\{\left(-v+t,-v^{\prime}\right) \mid t \in \mathbb{R}_{\geq 0}\right\} \cup\left\{\left(-v,-v^{\prime}+t\right) \mid t \in \mathbb{R}_{\geq 0}\right\} \cup\left\{\left(-v-t,-v^{\prime}-t\right) \mid t \in \mathbb{R}_{\geq 0}\right\}$.

It induces a fan $\Sigma\left(v, v^{\prime}\right)$ centered at $\left(-v,-v^{\prime}\right)$ with seven cones of various dimensions.

Let $v=\left(v_{1}, v_{1}^{\prime}, \ldots, v_{m}, v_{m}^{\prime}\right) \in \mathbb{R}^{2 m}$. The union of the spiders $\cup_{i=1}^{m} S\left(v_{i}, v_{i}^{\prime}\right)$ subdivides $\mathbb{R}^{2}$ into the union of 0,1 , and 2-dimensional convex cells (some of these cells are unbounded). We denote this convex subdivision of $\mathbb{R}^{2}$ by $\mathcal{S}(v)$.

Let $v, w \in \mathbb{R}^{2 m}$. We say that $\mathcal{S}(v)$ and $\mathcal{S}(w)$ are combinatorially equivalent if for all choices of cones $\sigma_{i} \in \Sigma\left(v_{i}, v_{i}^{\prime}\right), \tau_{i} \in \Sigma\left(w_{i}, w_{i}^{\prime}\right)$ such that $\sigma_{i}+\left(v_{i}, v_{i}^{\prime}\right)=\tau_{i}+\left(w_{i}, w_{i}^{\prime}\right)$, we have $\cap_{i=1}^{m} \sigma_{i} \neq \emptyset$ if and only if $\cap_{i=1}^{m} \tau_{i} \neq \emptyset$. We denote by $[\mathcal{S}(v)]$ the equivalence class of $\mathcal{S}(v)$. If $[\mathcal{S}(v)]=[\mathcal{S}(w)]$, then we say that two cells in $\mathcal{S}(v)$ and $\mathcal{S}(w)$ are corresponding if they can be written as $\cap_{i=1}^{m} \sigma_{i}$ and $\cap_{i=1}^{m} \tau_{i}$ where $\sigma_{i}+\left(v_{i}, v_{i}^{\prime}\right)=\tau_{i}+\left(w_{i}, w_{i}^{\prime}\right.$ ) for all $i$ (see Table 2 ).

Lemma 8.2. Intersections of cones $\sigma \in \mathcal{P}_{v}^{m}$ with $v+\mathbb{R}^{2} \cong \mathbb{R}^{2}$ give the subdivision $\mathcal{S}(v)$. In particular, if $v, w \in \mathbb{R}^{2 m}$ then $[\mathcal{S}(v)]=[\mathcal{S}(w)]$ if and only if $[v],[w] \in \mathbb{R}^{2 m} / \mathbb{R}^{2}$ lie in the relative interior of the same cone of the quotient fan $\mathcal{Q}_{m}$. 
TABLE 2. In the top row, an example of combinatorially equivalent subdivisions $\mathcal{S}(v)$ and $\mathcal{S}(w)$. Corresponding 2-cells are labeled with the same number. In the second row, an example of not combinatorially equivalent subdivisions.

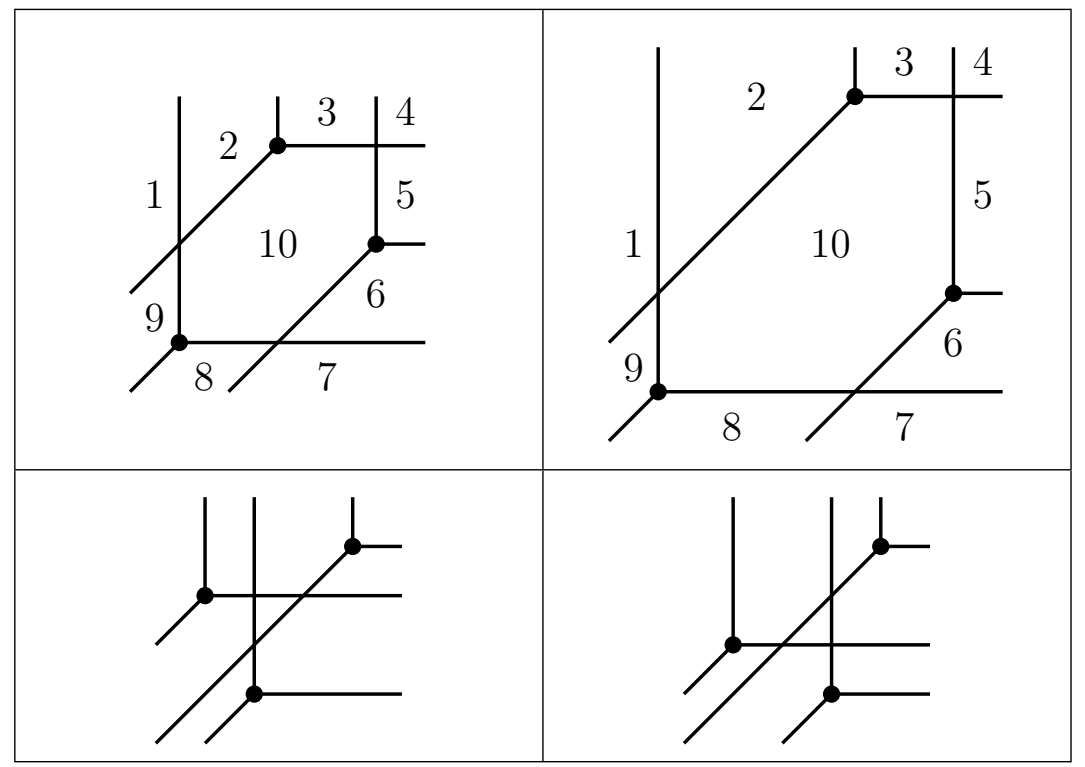

Proof. Let $C \subseteq \mathbb{R}^{2}$ be an arbitrary cell in $\mathcal{S}(v)$. Then for $i=1, \ldots, m$ there exist cones $\sigma_{i} \in \Sigma\left(v_{i}, v_{i}^{\prime}\right)$ such that $C=\cap_{i=1}^{m} \sigma_{i}$. Note that

$$
\tau=\left(\left(v_{1}, v_{1}^{\prime}\right)+\sigma_{1}\right) \times \cdots \times\left(\left(v_{m}, v_{m}^{\prime}\right)+\sigma_{m}\right)
$$

is a cone in the product fan $\mathcal{P}_{v}^{m}$. It is clear that $v+C=\left(v+\mathbb{R}^{2}\right) \cap \tau$.

Definition 8.3. Next we define the family over $Y_{\mathcal{Q}_{m}}$. Let $\mathcal{R}_{m}$ be the fan in $\mathbb{R}^{2 m}$ with cones $\eta=q^{-1}(\xi) \cap \tau$ for $\xi \in \mathcal{Q}_{m}$ and $\tau \in \mathcal{P}^{m}$.

Proposition 8.4. We have a commutative diagram of morphisms of toric varieties:

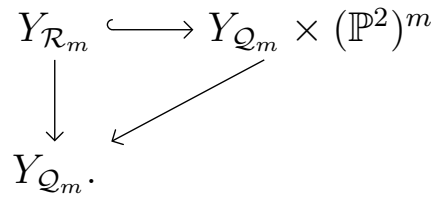

The top row is an embedding, and the toric morphism $Y_{\mathcal{R}_{m}} \rightarrow Y_{\mathcal{Q}_{m}}$ is flat with reduced fibers.

Proof. Consider the injective linear map given by

$$
\mathbb{R}^{2 m} \hookrightarrow\left(\mathbb{R}^{2 m} / \mathbb{R}^{2}\right) \times \mathbb{R}^{2 m}, \quad v \mapsto([v], v) .
$$

Then $\mathcal{R}_{m}$ is the restriction to $\mathbb{R}^{2 m}$ of the product fan $\mathcal{Q}_{m} \times \mathcal{P}^{m}$. By [Mol21, Theorem 2.1.4], in order to show that $Y_{\mathcal{R}_{m}} \rightarrow Y_{\mathcal{Q}_{m}}$ is flat with reduced fibers, we need to check that

(1) Every cone $\eta \in \mathcal{R}_{m}$ surjects onto a cone $\sigma \in \mathcal{Q}_{m}$;

(2) Whenever $q(\eta)=\sigma$, the lattice points in $\eta$ surject onto the lattice points in $\sigma$. 
TABLE 3. All combinatorial types of Mustafin triangles (up to symmetries) enumerating all cones in $\mathcal{Q}_{3}$. The table is from [CHSW11, Figure 6].

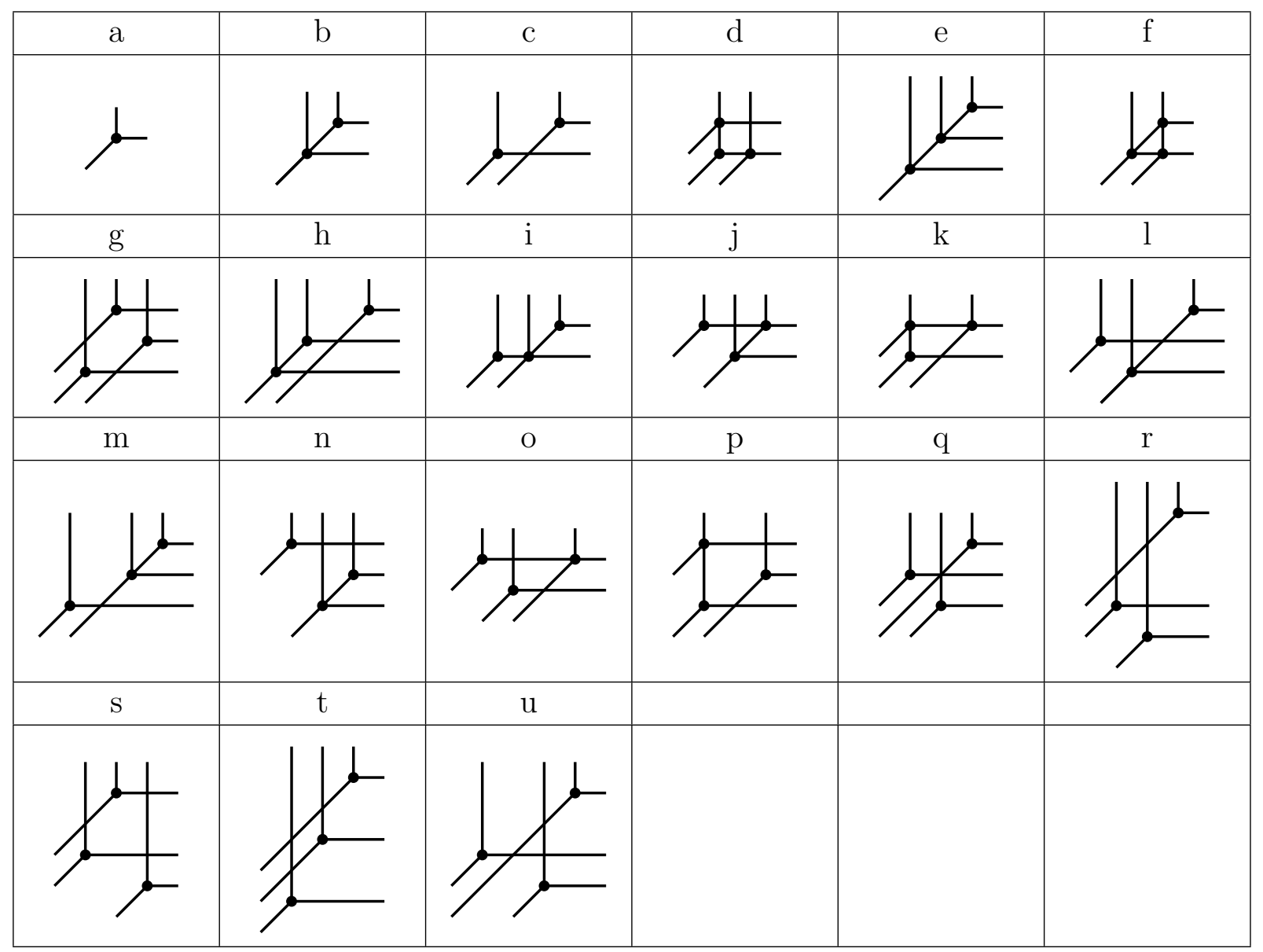

Let $\eta \in \mathcal{R}_{m}$. Then $\eta=q^{-1}(\xi) \cap \tau$ for some cones $\xi \in \mathcal{Q}_{m}$ and $\tau \in \mathcal{P}^{m}$. Thus $q(\eta)=\xi \cap q(\tau)$. By [KSZ91, §1], the quotient fan $\mathcal{Q}_{m}$ is the common refinement of the images in $\mathbb{R}^{2 m} / \mathbb{R}^{2}$ of cones in $\mathcal{P}^{m}$. For this reason, we have that $\xi \cap q(\tau)$ is a cone in $\mathcal{Q}_{m}$, proving part (1).

For part (2), let $\eta \in \mathcal{R}_{m}$ and let $[w] \in q(\eta)$ be a lattice point, which means that we can assume $w$ has integral coordinates. Write $\eta=q^{-1}(\xi) \cap \tau$ for $\xi \in \mathcal{Q}_{m}, \tau \in \mathcal{P}^{m}$, implying that $[w] \in \xi$ and $[w] \in q(\tau)$. Thus, $w \in q^{-1}(\xi)$ and $w+\left(x, x^{\prime}, \ldots, x, x^{\prime}\right) \in \tau$ for some $\left(x, x^{\prime}\right) \in \mathbb{R}^{2}$.

Write $\tau=\tau_{1} \times \cdots \times \tau_{m}$. Then $\left(x, x^{\prime}\right) \in \tau_{i}-\left(w_{i}, w_{i}^{\prime}\right)$ for all $i$. So $\left(x, x^{\prime}\right)$ is a point in the cell $C=\cap_{i=1}^{m}\left(\tau_{i}-\left(w_{i}, w_{i}^{\prime}\right)\right)$ of the subdivision $\mathcal{S}(w)$ of $\mathbb{R}^{2}$. Notice that $C$ always contains a point $\left(y, y^{\prime}\right)$ with integral coordinates. This is because, by the geometry of the fan $\mathcal{P}, C$ is a convex polytope with integral vertices and sides with slopes 0,1 , or vertical.

It follows that $w+\left(y, y^{\prime}, \ldots, y, y^{\prime}\right) \in \tau$ and therefore $w+\left(y, y^{\prime}, \ldots, y, y^{\prime}\right) \in q^{-1}(\xi) \cap \tau=\eta$ is a lattice point mapping to $[w]$.

Remark 8.5. One could adapt the above results for $Y^{m}$, where $Y$ is an arbitrary projective toric surface, except for Proposition 8.4, which used specific properties of the fan of $\mathbb{P}^{2}$. 
The conclusion of Proposition 8.4 holds for any projective toric surface such that the slopes of the rays of its associated fan are in the set $\{0,1, \infty\}$, i.e. for a toric del Pezzo surface.

Lemma 8.6. For $i \in\{1, \ldots, m\}$, we have "forgetful" toric morphisms of toric varieties

$$
Y_{\mathcal{Q}_{m}} \rightarrow Y_{\mathcal{Q}_{m-1}}
$$

induced by linear maps $p_{i}: \mathbb{R}^{2 m} \rightarrow \mathbb{R}^{2 m-2}, v \mapsto\left(v_{1}, v_{1}^{\prime}, \ldots, v_{i-1}, v_{i-1}^{\prime}, v_{i+1}, v_{i+1}^{\prime}, \ldots, v_{m}, v_{m}^{\prime}\right)$.

Proof. Let $v, w \in \mathbb{R}^{2 m}$. We claim that if $[\mathcal{S}(v)]=[\mathcal{S}(w)]$ then $\left[\mathcal{S}\left(p_{i}(v)\right)\right]=\left[\mathcal{S}\left(p_{i}(w)\right)\right]$ for all $i$. But this is immediate from Definition 8.1, for $j \in\{1, \ldots, m\} \backslash\{i\}$, consider cones $\sigma_{j} \in \Sigma\left(v_{j}, v_{j}^{\prime}\right), \tau_{j} \in \Sigma\left(w_{j}, w_{j}^{\prime}\right)$ such that $\sigma_{j}+\left(v_{j}, v_{j}^{\prime}\right)=\tau_{j}+\left(w_{j}, w_{j}^{\prime}\right)$. Assume that $\cap_{j \neq i} \sigma_{j} \neq \emptyset$. We want to show that $\bigcap_{j \neq i} \tau_{j} \neq \emptyset$. There exists $\sigma_{i} \in \Sigma\left(v_{i}, v_{i}^{\prime}\right)$ such that $\cap_{i} \sigma_{i} \neq \emptyset$ because $\Sigma\left(v_{i}, v_{i}^{\prime}\right)$ covers the entire $\mathbb{R}^{2}$. Let $\tau_{i} \in \Sigma\left(w_{i}, w_{i}^{\prime}\right)$ such that $\sigma_{i}+\left(v_{i}, v_{i}^{\prime}\right)=\tau_{i}+\left(w_{i}, w_{i}^{\prime}\right)$. Since $[\mathcal{S}(v)]=[\mathcal{S}(w)]$, we know that also $\cap_{j} \tau_{j} \neq \emptyset$, so $\cap_{j \neq i} \tau_{j} \neq \emptyset$, proving what we want.

Next we give a modular interpretation of $Y_{\mathcal{Q}_{m}}$ as the moduli space of reducible surfaces with marked points. The torus $\left(\mathbb{G}_{m}^{2}\right)^{m}$ is a configuration space of $m$ points $p_{1}, \ldots, p_{m} \in \mathbb{G}_{m}^{2}$. These light points are allowed to coincide. If we add three heavy points $e_{1}, e_{2}, e_{3}$, the quotient torus $\left(\mathbb{G}_{m}^{2}\right)^{m} / \mathbb{G}_{m}^{2}$ becomes isomorphic to the $\mathrm{PGL}_{3}$-orbit space for $m+3$ points

$$
e_{1}, e_{2}, e_{3}, p_{1}, \ldots, p_{m} \in \mathbb{P}^{2}
$$

such that any of the $m$ quadruples of the form $\left(p_{i}, e_{1}, e_{2}, e_{3}\right)$ is in linearly general position. To reduce to the torus action, we normalize $e_{1}, e_{2}, e_{3}$ to be the standard basis vectors. Given $K$-points $p_{1}, \ldots, p_{m} \in \mathbb{G}_{m}^{2}(K)$, we have $m$ lattices $L_{i}$ for $i=1, \ldots, m$ that stabilize quadruples of the form $\left(e_{1}, e_{2}, e_{3}, p_{i}\right)$ (some of these lattices can coincide). Notice that these lattices are in the same apartment that corresponds to the basis $e_{1}, e_{2}, e_{3}$.

Proposition 8.7. The family $Y_{\mathcal{R}_{m}} \rightarrow Y_{\mathcal{Q}_{m}}$ is the pullback of the universal family of the Hilbert scheme of $\left(\mathbb{P}^{2}\right)^{m}$. Let $\overline{\mathbf{a}}: \operatorname{Spec}(R) \rightarrow Y_{\mathcal{Q}_{m}}$ be a morphism such that $\overline{\mathbf{a}}(\operatorname{Spec} K)$ is contained in the quotient torus. Then $\overline{\mathbf{a}}^{*} Y_{\mathcal{R}_{m}}$ is isomorphic to the Mustafin join $\mathbb{P}\left(L_{1}, \ldots, L_{m}\right)$.

Proof. The first part follows from Proposition 8.4. For the second part, write $\overline{\mathbf{a}}(\operatorname{Spec} K)=$ $\left(a_{1}, \ldots, a_{m}\right) \bmod \mathbb{G}_{m}^{2}$, an element of $\left(\mathbb{G}_{m}^{2}\right)^{m} / \mathbb{G}_{m}^{2}(K)$. We have that $\left(\left.\overline{\mathbf{a}}\right|_{\operatorname{Spec} K}\right)^{*} Y_{\mathcal{R}_{m}} \cong \mathbb{P}_{K}^{2}$ is embedded in $\left(\mathbb{P}_{K}^{2}\right)^{m}$ by the diagonal embedding of $\mathbb{P}_{K}^{2}$ followed by the automorphism of $\left(\mathbb{P}_{K}^{2}\right)^{m}$ induced by the action of $\left(a_{1}^{-1}, \ldots, a_{m}^{-1}\right)$. The morphism $Y_{\mathcal{R}_{m}} \rightarrow Y_{\mathcal{Q}_{m}}$ is flat by Proposition 8.4. so $\overline{\mathbf{a}}^{*} Y_{\mathcal{R}_{m}}$ equals the Zariski closure of $\mathbb{P}_{K}^{2}$ in $\left(\mathbb{P}_{R}^{2}\right)^{m}$ under the above embedding. This shows that $\overline{\mathbf{a}}^{*} Y_{\mathcal{R}_{m}}$ is equal to the Mustafin join of the lattices $L_{i}=a_{i 1} e_{1} R+a_{i 2} e_{2} R+a_{i 3} e_{3} R$, where we view $a_{i}$ as the point in $\mathbb{P}^{2}$ given by $\left[a_{i 1}: a_{i 2}: a_{i 3}\right]$.

Definition 8.8. Let $\lambda_{i}$ to be the map of lattices $\mathbb{Z}^{2 m} / \mathbb{Z}^{2} \rightarrow \mathbb{Z}^{2 m}$ given by

$$
[v] \mapsto\left(v_{1}-v_{i}, v_{1}^{\prime}-v_{i}^{\prime}, \ldots, v_{m}-v_{i}, v_{m}^{\prime}-v_{i}^{\prime}\right) .
$$

Each of these maps is a section of the quotient map $q: \mathbb{Z}^{2 m} \rightarrow \mathbb{Z}^{2 m} / \mathbb{Z}^{2}$.

Proposition 8.9. The family $Y_{\mathcal{R}_{m}} \rightarrow Y_{\mathcal{Q}_{m}}$ admits $m$ "light" sections $\ell_{1}, \ldots, \ell_{m}$, which are toric morphisms induced by the linear maps $\lambda_{1}, \ldots, \lambda_{m}$, and three "heavy" constant sections $t_{1}, t_{2}, t_{3}$ given by $t_{i}=Y_{\mathcal{Q}_{m}} \times\left\{e_{i}\right\} \times \ldots \times\left\{e_{i}\right\} \subseteq Y_{\mathcal{Q}_{m}} \times\left(\mathbb{P}^{2}\right)^{m}$ for $i=1,2,3$ (see Proposition 8.4). 
Proof. We need to show that each of the maps $\lambda_{i}$ induces a map of fans from $\mathcal{Q}_{m}$ to $\mathcal{R}_{m}$. Suppose $[v],[w] \in \mathbb{R}^{2 m} / \mathbb{R}^{2}$ are contained in the relative interior of a cone $\sigma$ of $\mathcal{Q}_{m}$. In order to show that $\lambda_{i}(\sigma)$ is contained in a cone of $\mathcal{R}_{m}$, it suffices to show that $\lambda_{i}([v]), \lambda_{i}([w])$ are contained in the same cone of $\mathcal{P}^{m}$, i.e. that $\left(v_{j}-v_{i}, v_{j}^{\prime}-v_{i}^{\prime}\right)$ and $\left(w_{j}-w_{i}, w_{j}^{\prime}-w_{i}^{\prime}\right)$ are contained in the same cone of $\mathcal{P}$ for every $j$. Note that the cone of $\mathcal{P}$ containing $\left(v_{j}-v_{i}, v_{j}^{\prime}-v_{i}^{\prime}\right)\left(\right.$ resp. $\left.\left(w_{j}-w_{i}, w_{j}^{\prime}-w_{i}^{\prime}\right)\right)$ is completely determined by the relative position of the spiders $S\left(v_{i}, v_{i}^{\prime}\right)$ and $S\left(v_{j}, v_{j}^{\prime}\right)$ (resp. $S\left(w_{i}, w_{i}^{\prime}\right)$ and $S\left(w_{j}, w_{j}^{\prime}\right)$ ). Since $\mathcal{S}(v)$ and $\mathcal{S}(w)$ are combinatorially equivalent, the claim follows.

Corollary 8.10. Fibers of the family $Y_{\mathcal{R}_{m}} \rightarrow Y_{\mathcal{Q}_{m}}$ can be computed as in Remark 5.3. A spider decomposition $S(v)$ induces a regular mixed polyhedral subdivision of $m \Delta_{2}$. The corresponding fiber is a broken toric variety with irreducible components that correspond to 2-cells of the subdivision of $m \Delta_{2}$. Three heavy sections correspond to vertices of $m \Delta_{2}$. Every light section $\ell_{i}$ lies in the irreducible component that corresponds to the spider $S\left(v_{i}, v_{i}^{\prime}\right)$.

Proof. After our discussion so far, the only claim needing proof is the one about light sections. We can reduce this claim to considering a one-parameter family a: $\operatorname{Spec}(R) \rightarrow Y_{\mathcal{Q}_{m}}$ with $\mathbf{a}(\operatorname{Spec}(K))$ contained in the quotient torus. Let us prove the statement for $\ell_{1}$, which, without loss of generality, we can assume equal to $[1: 1: 1]$. In particular, we have that $L_{1}=e_{1} R+$ $e_{2} R+e_{3} R$. Let us rescale the points $\left[a_{j 1}: a_{j 2}: a_{j 3}\right], j \geq 2$, so that val $\left(a_{j 1}\right), \operatorname{val}\left(a_{j 2}\right), \operatorname{val}\left(a_{j 3}\right) \leq$ 0 , with equality achieved at least once for each $j$. Then $v=(1,1,1) \in L_{j} \backslash t L_{j}$ for all $j=1, \ldots, m$. Moreover, $L_{1}$ is contained in the intersection $L_{1} \cap \ldots \cap L_{m}$. Therefore, by Lemma 5.7 we have that the limit of $\ell_{i}$ in the central fiber of the Mustafin join $\mathbb{P}\left(L_{1}, \ldots, L_{m}\right)$ is a smooth point contained in the primary component corresponding to $L_{1}$, which is the one corresponding to the $i$-th spider.

Example 8.11. Let us consider the arc

$$
\mathbf{a}=\left(e_{1}=[1: 0: 0], e_{2}=[0: 1: 0], e_{3}=[0: 0: 1], a_{1}=[1: 1: 1], a_{2}=\left[t^{2}: 1: t\right]\right) .
$$

We studied the 5-pointed degeneration of $\mathbb{P}^{2}$ parametrized by $\overline{\mathbf{a}}(0) \in \overline{\mathbf{X}}_{\mathrm{GP}}(3,5)$ in Example 6.2. We now compute the 5-pointed fiber of $Y_{\mathcal{R}_{2}} \rightarrow Y_{\mathcal{Q}_{2}}$ over the limit point $\overline{\mathbf{a}}(0) \in Y_{\mathcal{Q}_{2}}$. By Proposition 8.7, such fiber is the central fiber of the Mustafin join of the following two lattices:

$$
L_{1}=e_{1} R+e_{2} R+e_{3} R, L_{2}=t^{2} e_{1} R+e_{2} R+t e_{3} R
$$

We can compute $\mathbb{P}\left(L_{1}, L_{2}\right)_{\mathbb{k}}$ using Remark 5.3 , and the result is pictured in Figure 13 . Note that the limit of $a_{i}$ lies in the primary component corresponding to $L_{i}$ by Corollary 8.10 .

\section{Toric analogue of $\overline{\mathbf{X}}_{\mathrm{GP}}(3, n)$}

We will use the toric Kapranov spaces $Y_{\mathcal{Q}_{m}}$ in $\$ 11$ to construct open patches of the Kapranov space $\overline{\mathbf{X}}(3, n)$ that cover an open planar locus with toroidal singularities. Its preimage in the Gerrizen-Piwek space $\overline{\mathbf{X}}_{\mathrm{GP}}(3, n)$ will be described using toric Gerrizen-Piwek spaces $Y_{\widetilde{\mathcal{Q}}_{m}}$ introduced in this section. While the same arc gives different fibers of the family $Y_{\mathcal{R}_{m}}$ (see Example 8.11) and the Gerrizen-Piwek family (see Example 6.2), under some conditions, which we investigate in this and the following sections, the Gerrizen-Piwek fiber can 


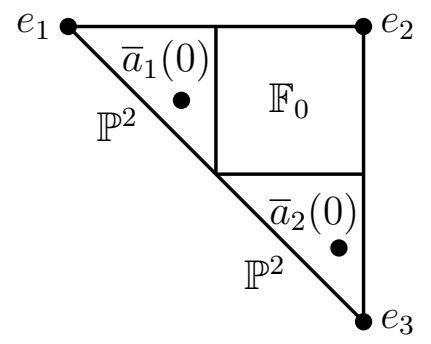

FiguRE 13. Fiber of $Y_{\mathcal{R}_{2}} \rightarrow Y_{\mathcal{Q}_{2}}$ over $\overline{\mathbf{a}}(0)$ in Example 8.11 .

be reconstructed from the fiber in $Y_{\mathcal{R}_{m}}$. To motivate the construction, write $n=m+3$ and take an arc

$$
a=\left(e_{1}, e_{2}, e_{3}, a_{1}, \ldots, a_{m}\right) \in\left(\mathbb{P}^{2}\right)^{n}(K)
$$

in linearly general position. Consider an apartment $A$ that corresponds to the basis $e_{1}, e_{2}, e_{3}$. By Remark 5.3, a lattice $t^{\alpha} e_{1} R+t^{\beta} e_{2} R+t^{\gamma} e_{3} R$ in the apartment corresponds to a lattice point $(-a,-b)=(\gamma-\alpha, \gamma-\beta) \in \mathbb{R}^{2}$. It is the 0 -cell of the spider $S(a, b)$.

Definition 9.1. The dual spider is defined as follows:

$S(a, b)^{\vee}=\left\{(-a-t,-b) \mid t \in \mathbb{R}_{\geq 0}\right\} \cup\left\{(-a,-b-t) \mid t \in \mathbb{R}_{\geq 0}\right\} \cup\left\{(-a+t,-b+t) \mid t \in \mathbb{R}_{\geq 0}\right\}$.

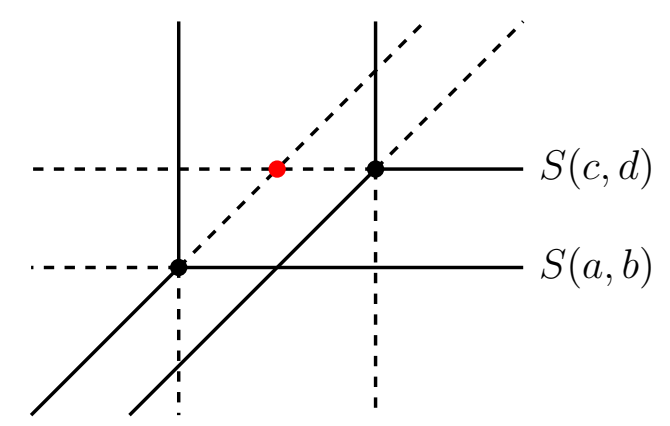

FiguRE 14. Intersection point of dashed dual spiders $S(a, b)^{\vee}$ and $S(c, d)^{\vee}$.

Lemma 9.2. Consider the stable lattices $L_{i}$ (resp. $\left.\bar{L}_{\alpha \beta, i j}\right)$ stabilizing quadruples $\left(e_{1}, e_{2}, e_{3}, a_{i}\right)$ for $i=1, \ldots, m$ (resp. $\left(e_{\alpha}, e_{\beta}, a_{i}, a_{j}\right)$ for $\left.\alpha, \beta=1,2,3, i, j=1, \ldots, m\right)$. The lattices $L_{i}$ belong to the apartment $A$ and are given by the integral points $\left(-v_{i},-v_{i}^{\prime}\right)$ in $\mathbb{R}^{2}$. A lattice $\bar{L}_{\alpha \beta, i j}$ does not have to be in the apartment $A$, but if it does, then it is the lattice $L_{\alpha \beta, i j}$ that corresponds to the integral point $\left(-x_{\alpha \beta, i j},-x_{\alpha \beta, i j}^{\prime}\right)$ from Table 4. If this point is different from $\left(-v_{i},-v_{i}^{\prime}\right)$ and $\left(-v_{j},-v_{j}^{\prime}\right)$ then it is the intersection point $(-a,-b)$ of the dual spiders $S\left(v_{i}, v_{i}^{\prime}\right)^{\vee}$ and $S\left(v_{j}, v_{j}^{\prime}\right)^{\vee}$.

Proof. Let $i, j \in\{1, \ldots, m\}$ be distinct indices and consider the stable lattices $L_{i}, L_{j}$. All their possible reciprocal positions as points $\left(-v_{i},-v_{i}^{\prime}\right),\left(-v_{j},-v_{j}^{\prime}\right)$ in $\mathbb{R}^{2}$ are listed in Table 4 . Let us determine the integral points $\left(x_{\alpha \beta, i j}, x_{\alpha \beta, i j}^{\prime}\right)$. 
Assume that the reciprocal position of $L_{i}, L_{j}$ is as shown in the first entry of the first row of Table 4. Then the limits of the points $p_{1}, p_{2}, p_{3}, a_{i}, a_{j}$ in the central fiber of the Mustafin join $\mathbb{P}\left(L_{i}, L_{j}\right)$ are shown in the left side of Figure 15 . We can then directly compute that

$$
\left(x_{12, i j}, x_{12, i j}^{\prime}\right)=\left(v_{j}, v_{j}^{\prime}\right),\left(x_{13, i j}, x_{13, i j}^{\prime}\right)=\left(v_{i}, v_{i}^{\prime}\right),\left(x_{23, i j}, x_{23, i j}^{\prime}\right)=\left(v_{j}, v_{j}^{\prime}\right) .
$$

An analogous argument applies to the remaining cases in the first two rows of Table 4.

Now assume that the reciprocal position of $L_{i}, L_{j}$ is as shown in the first entry of the third row of Table 4 instead. Define $L_{i j}$ as the lattice corresponding to the intersection point $(-a,-b)$ of the dual spiders $S\left(v_{i}, v_{i}^{\prime}\right)^{\vee}$ and $S\left(v_{j}, v_{j}^{\prime}\right)^{\vee}$. Then the limits of the points $p_{1}, p_{2}, p_{3}, a_{i}, a_{j}$ in the central fiber of the Mustafin join $\mathbb{P}\left(L_{i}, L_{j}, L_{i j}\right)$ can be found in the right side of Figure 15. From this we can directly compute that

$$
\left(x_{12, i j}, x_{12, i j}^{\prime}\right)=\left(v_{i}, v_{i}^{\prime}\right),\left(x_{13, i j}, x_{13, i j}^{\prime}\right)=(a, b),\left(x_{23, i j}, x_{23, i j}^{\prime}\right)=\left(v_{j}, v_{j}^{\prime}\right) .
$$

The remaining cases in the last two rows of Table 4 are handled in an analogous way.
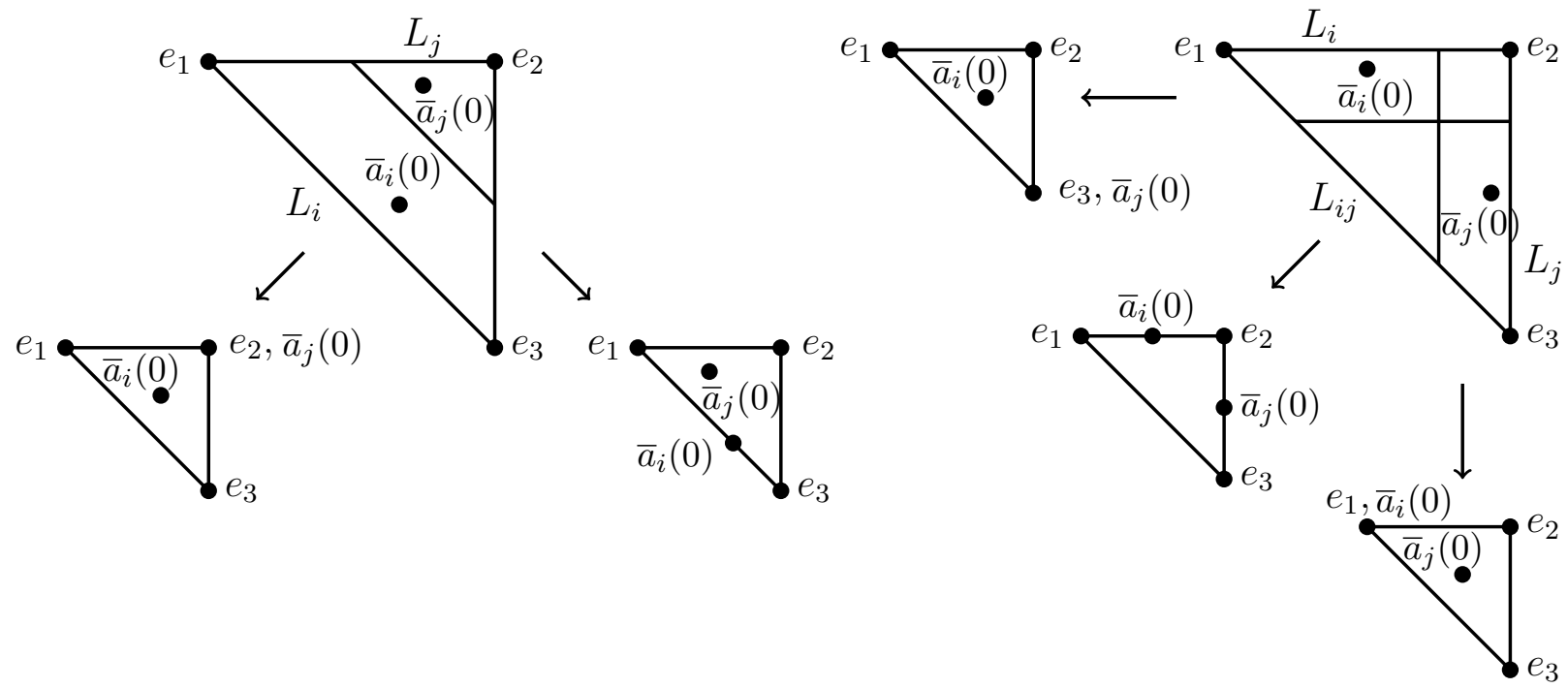

Figure 15. In the proof of Lemma 9.2, the central fibers of the Mustafin joins $\mathbb{P}\left(L_{i}, L_{j}\right), \mathbb{P}\left(L_{i}, L_{j}, L_{i j}\right)$ and the limits of $e_{1}, e_{2}, e_{3}, a_{i}, a_{j}$.

Definition 9.3. We let

$$
N=\left(\begin{array}{l}
3 \\
2
\end{array}\right)\left(\begin{array}{c}
m \\
2
\end{array}\right)
$$

Let $\pi: \mathbb{R}^{2 m+2 N} \rightarrow \mathbb{R}^{2 m}$ be the projection map and let $\iota: \mathbb{R}^{2 m} \rightarrow \mathbb{R}^{2 m+2 N}$ be the function that sends a vector $v$ to the vector $(v, x)$ such that the components $x_{\alpha \beta, i j}, x_{\alpha \beta, i j}^{\prime}$ of the vector $x$ are given in Table 4 . The induced maps $\mathbb{R}^{2 m+2 N} / \mathbb{R}^{2} \rightarrow \mathbb{R}^{2 m} / \mathbb{R}^{2}$ and $\mathbb{R}^{2 m} / \mathbb{R}^{2} \rightarrow \mathbb{R}^{2 m+2 N} / \mathbb{R}^{2}$ are well-defined and we denote them by $\bar{\pi}$ and $\bar{\iota}$, respectively. We define

$$
\widetilde{\mathcal{S}}(v)=\mathcal{S}(\iota(v)),
$$


TABLE 4. The red dot (intersection of dual spiders) is in position $(-a,-b) \in$ $\mathbb{R}^{2}$.

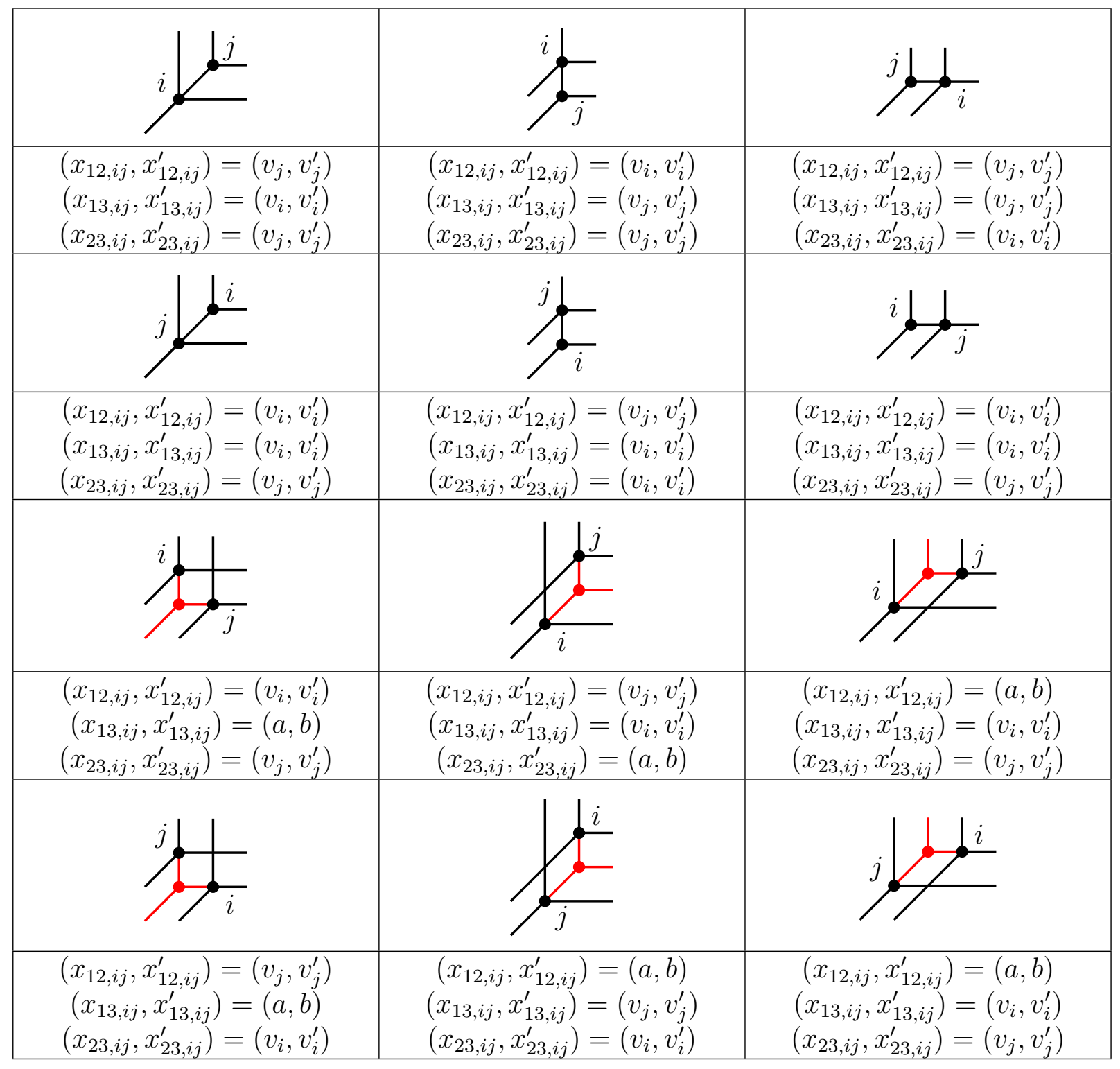

see Figure 16 for an example. The subdivision $\widetilde{\mathcal{S}}(v)$ refines $\mathcal{S}(v)$.

We note that the image of $\iota$ consists of entire cones of the fan $\mathcal{Q}_{m+N}$ :

Lemma 9.4. Let $v \in \mathbb{R}^{2 m}, z \in \mathbb{R}^{2 m+2 N}$. If $[\mathcal{S}(\iota(v))]=[\mathcal{S}(z)]$, then $z=\iota(\pi(z))$.

Proof. Write $\iota(v)=(v, x), z=(\pi(z), y)$, where

$$
x=\left(\ldots, x_{\alpha \beta, i j}, x_{\alpha \beta, i j}^{\prime}, \ldots\right), y=\left(\ldots, y_{\alpha \beta, i j}, y_{\alpha \beta, i j}^{\prime}, \ldots\right) .
$$



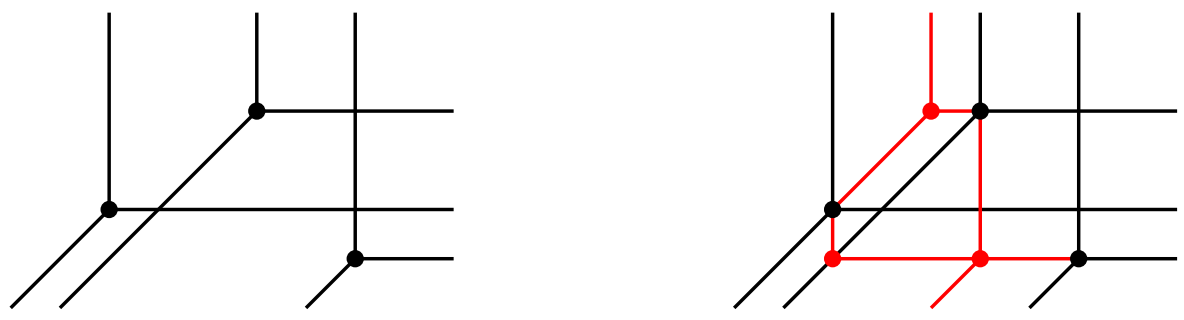

Figure 16. Example of $\mathcal{S}(v)$ (on the left) and its refinement $\widetilde{\mathcal{S}}(v)$ (on the right).

Recall that the pairs $\left(x_{\alpha \beta, i j}, x_{\alpha \beta, i j}^{\prime}\right)$ are defined according to Definition 9.3. Fix distinct indices $\alpha, \beta \in[3], i, j \in[m]$, and for simplicity of notation set $v_{i j}=\left(v_{i}, v_{i}^{\prime}, v_{j}, v_{j}^{\prime}\right), z_{i j}=\left(z_{i}, z_{i}^{\prime}, z_{j}, z_{j}^{\prime}\right)$. Note that the hypothesis $[\mathcal{S}(\iota(v))]=[\mathcal{S}(z)]$ implies that

$$
\left[\mathcal{S}\left(v_{i j}, x_{\alpha \beta, i j}, x_{\alpha \beta, i j}^{\prime}\right)\right]=\left[\mathcal{S}\left(z_{i j}, y_{\alpha \beta, i j}, y_{\alpha \beta, i j}^{\prime}\right)\right] .
$$

Based on the definition of $\iota(v)$, we have the following three cases on $\left(x_{\alpha \beta, i j}, x_{\alpha \beta, i j}^{\prime}\right)$ :

If $\left(x_{\alpha \beta, i j}, x_{\alpha \beta, i j}^{\prime}\right)=\left(v_{i}, v_{i}^{\prime}\right)$, then we show that $\left(y_{\alpha \beta, i j}, y_{\alpha \beta, i j}^{\prime}\right)=\left(z_{i}, z_{i}^{\prime}\right)$. Up to symmetries, we have two possibilities for the reciprocal position of the points $\left(-v_{i},-v_{i}^{\prime}\right),\left(-v_{j},-v_{j}^{\prime}\right)$ in $\mathbb{R}^{2}$. Say we are in the first case of the first row of Table 4. Consider the cones $\sigma_{i}=\left\{\left(-v_{i},-v_{i}^{\prime}\right)\right\} \in$ $\Sigma\left(v_{i}, v_{i}^{\prime}\right), \sigma_{j} \in \Sigma\left(v_{j}, v_{j}^{\prime}\right), \sigma=\left\{\left(-x_{\alpha \beta, i j},-x_{\alpha \beta, i j}^{\prime}\right)\right\} \in \Sigma\left(x_{\alpha \beta, i j}, x_{\alpha \beta, i j}^{\prime}\right)$ such that $\sigma_{i} \cap \sigma_{j} \cap \sigma=$ $\left\{\left(-v_{i},-v_{i}^{\prime}\right)\right\}$ and $\sigma_{j}$ is a ray. Consider the corresponding cones $\tau_{i}=\left\{\left(-z_{i},-z_{i}^{\prime}\right)\right\} \in \Sigma\left(z_{i}, z_{i}^{\prime}\right)$, $\tau_{j} \in \Sigma\left(z_{j}, z_{j}^{\prime}\right), \tau=\left\{\left(-y_{\alpha \beta, i j},-y_{\alpha \beta, i j}^{\prime}\right)\right\} \in \Sigma\left(y_{\alpha \beta, i j}, y_{\alpha \beta, i j}^{\prime}\right)$. Then also $\tau_{i} \cap \tau_{j} \cap \tau \neq \emptyset$ by (6), and hence this intersection has to be equal to $\left\{\left(-z_{i},-z_{i}^{\prime}\right)\right\}$, showing that $\left(y_{\alpha \beta, i j}, y_{\alpha \beta, i j}^{\prime}\right)=\left(z_{i}, z_{i}^{\prime}\right)$. The second possibility for the reciprocal position of $\left(-v_{i},-v_{i}^{\prime}\right),\left(-v_{j},-v_{j}^{\prime}\right)$, up to symmetry, is the first case of the third row of Table 4 . Here we use the same argument as in the previous case, but we take $\sigma_{j}$ to be 2-dimensional instead.

If $\left(x_{\alpha \beta, i j}, x_{\alpha \beta, i j}^{\prime}\right)=\left(v_{j}, v_{j}^{\prime}\right)$, then we show that $\left(y_{\alpha \beta, i j}, y_{\alpha \beta, i j}^{\prime}\right)=\left(z_{j}, z_{j}^{\prime}\right)$ by an analogous argument to the one above.

Finally, assume $\left(x_{\alpha \beta, i j}, x_{\alpha \beta, i j}^{\prime}\right)$ is the unique intersection point $(-a,-b)$ of the dual spi$\operatorname{ders} S\left(v_{i}, v_{i}^{\prime}\right)^{\vee}, S\left(v_{j}, v_{j}^{\prime}\right)^{\vee}$. Consider the cones given by $\sigma_{i} \in \Sigma\left(v_{i}, v_{i}^{\prime}\right), \sigma_{j} \in \Sigma\left(v_{j}, v_{j}^{\prime}\right), \sigma=$ $\left\{\left(-x_{\alpha \beta, i j},-x_{\alpha \beta, i j}^{\prime}\right)\right\} \in \Sigma\left(x_{\alpha \beta, i j}, x_{\alpha \beta, i j}^{\prime}\right)$ such that $\sigma_{i} \cap \sigma_{j} \cap \sigma=\{(-a,-b)\}$ and $\sigma_{i}, \sigma_{j}$ are rays. Consider the corresponding cones $\tau_{i} \in \Sigma\left(z_{i}, z_{i}^{\prime}\right), \tau_{j} \in \Sigma\left(z_{j}, z_{j}^{\prime}\right), \tau=\left\{\left(-y_{\alpha \beta, i j},-y_{\alpha \beta, i j}^{\prime}\right)\right\} \in$ $\Sigma\left(y_{\alpha \beta, i j}, y_{\alpha \beta, i j}^{\prime}\right)$. Then $\tau_{i} \cap \tau_{j} \cap \tau \neq \emptyset$ by (6), and hence $\left(-y_{\alpha \beta, i j},-y_{\alpha \beta, i j}^{\prime}\right)$ is the unique intersection point of the dual spiders $S\left(z_{i}, z_{i}^{\prime}\right)^{\vee}, S\left(z_{j}, z_{j}^{\prime}\right)^{\vee}$.

Lemma-Definition 9.5. There is a unique complete fan $\widetilde{\mathcal{Q}}_{m}$ in $\mathbb{R}^{2 m} / \mathbb{R}^{2}$ such that $\bar{\iota}$ is linear on every cone $\sigma$ of $\widetilde{\mathcal{Q}}_{m}$ and $\bar{\iota}(\sigma)$ is a cone of the quotient fan $\mathcal{Q}_{m+N}$ in $\mathbb{R}^{2 m+2 N} / \mathbb{R}^{2}$. Vectors $[v],[w] \in \mathbb{R}^{2 m} / \mathbb{R}^{2}$ lie in the relative interior of the same cone $\sigma$ of the fan $\widetilde{\mathcal{Q}}_{m}$ if and only if $[\widetilde{\mathcal{S}}(v)]=[\widetilde{\mathcal{S}}(w)]$. The collection of cones $\bar{\iota}(\sigma)$ is a subfan $\mathcal{Q}_{m+N}^{\prime}$ of $\mathcal{Q}_{m+N}$ of cones corresponding to subdivisions $\mathcal{S}(z), z \in \mathbb{R}^{2 m+2 N}$, such that $\mathcal{S}(z)=\widetilde{\mathcal{S}}(\pi(z))$.

Proof. Given $v \in \mathbb{R}^{2 m}$ and two distinct indices $i, j \in[m]$, then the possible reciprocal positions for $\left(-v_{i},-v_{i}^{\prime}\right),\left(-v_{j},-v_{j}^{\prime}\right) \in \mathbb{R}^{2}$, if they are distinct points, are pictured in Table 4 . Each 
possibility is described by a set of inequalities and/or equalities. Therefore, given $v \in \mathbb{R}^{2 m}$, for each pair of distinct indices $i, j \in[m]$ we have a set of inequalities and/or equalities, and considering all these together defines a convex cone $\sigma_{v} \subseteq \mathbb{R}^{2 m}$. (Clearly, if $w$ is in the relative interior of $\sigma_{v}$, then $\sigma_{w}=\sigma_{v}$.) We denote by $\mathcal{W}$ the collection of all these cones in $\mathbb{R}^{2 m}$. We have that $\iota$ is linear on each cone in $\mathcal{W}$ because each $\left(x_{\alpha \beta, i j}, x_{\alpha \beta, i j}^{\prime}\right)$ is linear by its definition. Note that given $v \in \mathbb{R}^{2 m}$ and $(a, b) \in \mathbb{R}^{2}$, then $v$ and $v+(a, b, \ldots, a, b)$ satisfy the same set of inequalities. Hence, the collection $q(\mathcal{W})$ of images of cones in $\mathcal{W}$ under the quotient $q: \mathbb{R}^{2 m} \rightarrow \mathbb{R}^{2 m} / \mathbb{R}^{2}$ is a collection of cones on which $\bar{\iota}$ is linear.

We now define $\mathcal{Q}_{m+N}^{\prime}$ to be the collection of cones $\sigma \in \mathcal{Q}_{m+N}$ such that $\bar{\iota}([v])$ is in the relative interior of $\sigma$ for some $[v] \in \mathbb{R}^{2 m} / \mathbb{R}^{2}$. Note that a cone $\sigma \in \mathcal{Q}_{m+N}^{\prime}$ is entirely contained in the image of $\bar{\iota}$ by Lemma 9.4. Moreover, $\bar{\iota}$ is linear on $(\bar{\iota})^{-1}(\sigma)$. To prove this, it is enough to show the following. Let $\bar{\iota}([v])$ be in the relative interior of $\sigma$ and let $[z]$ be any other point in the relative interior of $\sigma$. We want to show that $\bar{\iota}$ is linear on the 2-dimensional cone generated by $\bar{\pi}([z])$ and $[v]$. Equivalently, $\iota$ is linear on the 2-dimensional cone generated by $\pi(z)$ and $v$. But this is clear because $z=\iota(\pi(z))$ by Lemma 9.4, and $\pi(z), v$ belong to the same cone of $\mathcal{W}$ because $[\mathcal{S}(z)]=[\mathcal{S}(\iota(v))]$.

Next, we observe that $\mathcal{Q}_{m+N}^{\prime}$ is a fan. Consider $\sigma \in \mathcal{Q}_{m+N}^{\prime}$, and let $\varphi \subseteq \sigma$ be a face. By the argument above, $\sigma$ is contained in the image of $\bar{\iota}$, hence also $\varphi$ is, so we can certainly find $[v] \in \mathbb{R}^{2 m} / \mathbb{R}^{2}$ such that $\bar{\imath}([v])$ is in the relative interior of $\varphi$. Combined with the fact that $\varphi \in \mathcal{Q}_{m+N}$ because $\mathcal{Q}_{m+N}$ is a fan, we can conclude that $\varphi \in \mathcal{Q}_{m+N}^{\prime}$.

We then define $\widetilde{\mathcal{Q}}_{m}$ to be the collection of preimages of cones in $\mathcal{Q}_{m+N}^{\prime}$ under $\bar{\iota}$. By the discussion above, $\widetilde{\mathcal{Q}}_{m}$ is a fan, and it is clearly complete.

We conclude with the combinatorial interpretation of the cones in $\widetilde{\mathcal{Q}}_{m}$. Let $[v],[w] \in$ $\mathbb{R}^{2 m} / \mathbb{R}^{2}$ be in the relative interior of the same cone $(\bar{\iota})^{-1}(\sigma) \in \widetilde{\mathcal{Q}}_{m}$, where $\sigma \in \mathcal{Q}_{m+N}^{\prime}$. Then $\bar{\iota}([v]), \bar{\iota}([w])$ are in the relative interior of $\sigma$, implying that $[\mathcal{S}(\iota(v))]=[\mathcal{S}(\iota(w))]$. Conversely, assume that $[\mathcal{S}(\iota(v))]=[\mathcal{S}(\iota(w))]$. This means that $\bar{\iota}([v]), \bar{\iota}([w])$ are in the relative interior of the same cone $\sigma \in \mathcal{Q}_{m+N}$, which by definition is also a cone of $\mathcal{Q}_{m+N}^{\prime}$. Hence, $[v],[w] \in$ $(\bar{\iota})^{-1}(\sigma) \in \widetilde{\mathcal{Q}}_{m}$, proving the last statement.

Definition 9.6. The variety $Y_{\widetilde{\mathcal{Q}}_{m}}$ is called the toric Gerritzen-Piwek space.

Proposition 9.7. There is a birational toric morphism $Y_{\widetilde{\mathcal{Q}}_{m}} \rightarrow Y_{\mathcal{Q}_{m}}$, a "forgetful" toric morphism $Y_{\widetilde{\mathcal{Q}}_{m}} \rightarrow Y_{\widetilde{\mathcal{Q}}_{[m] \backslash\{i\}}}$ for each $i=1, \ldots, m$ and a commutative diagram

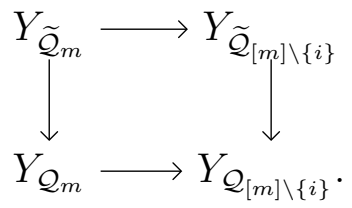

Proof. We claim that the fan $\widetilde{\mathcal{Q}}_{m}$ refines the fan $\mathcal{Q}_{m}$. We need to show that if $v, w \in \mathbb{R}^{2 m}$ and $[\widetilde{\mathcal{S}}(v)]=[\widetilde{\mathcal{S}}(w)]$ then $[\mathcal{S}(v)]=[\mathcal{S}(w)]$. But this is a consequence of Lemma 8.6.

For the second statement, let $v, w \in \mathbb{R}^{2 m}$ and consider the projections $p_{i}: \mathbb{R}^{2 m} \rightarrow \mathbb{R}^{2 m-2}$ for $i=1, \ldots, m$. We claim that if $\widetilde{\mathcal{S}}(v)$ and $\widetilde{\mathcal{S}}(w)$ are combinatorially equivalent, then 
$\widetilde{\mathcal{S}}\left(p_{i}(v)\right)$ and $\widetilde{\mathcal{S}}\left(p_{i}(w)\right)$ are combinatorial equivalent. Write $\widetilde{\mathcal{S}}(v)=\mathcal{S}(v, x), \widetilde{\mathcal{S}}(w)=\mathcal{S}(w, y)$

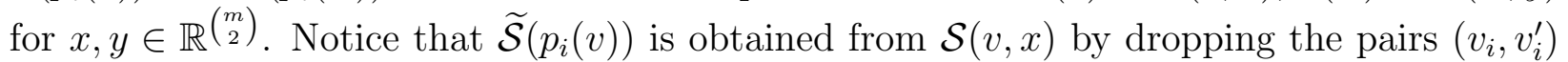
and $\left(x_{i j}, x_{i j}^{\prime}\right)$ for $j \neq i$, and similarly for $\widetilde{\mathcal{S}}\left(p_{i}(w)\right)$. It remains to apply Lemma 8.6 .

TABle 5. Decompositions $\widetilde{\mathcal{S}}(v)$ obtained from decompositions $\mathcal{S}(v)$ of Table 3.

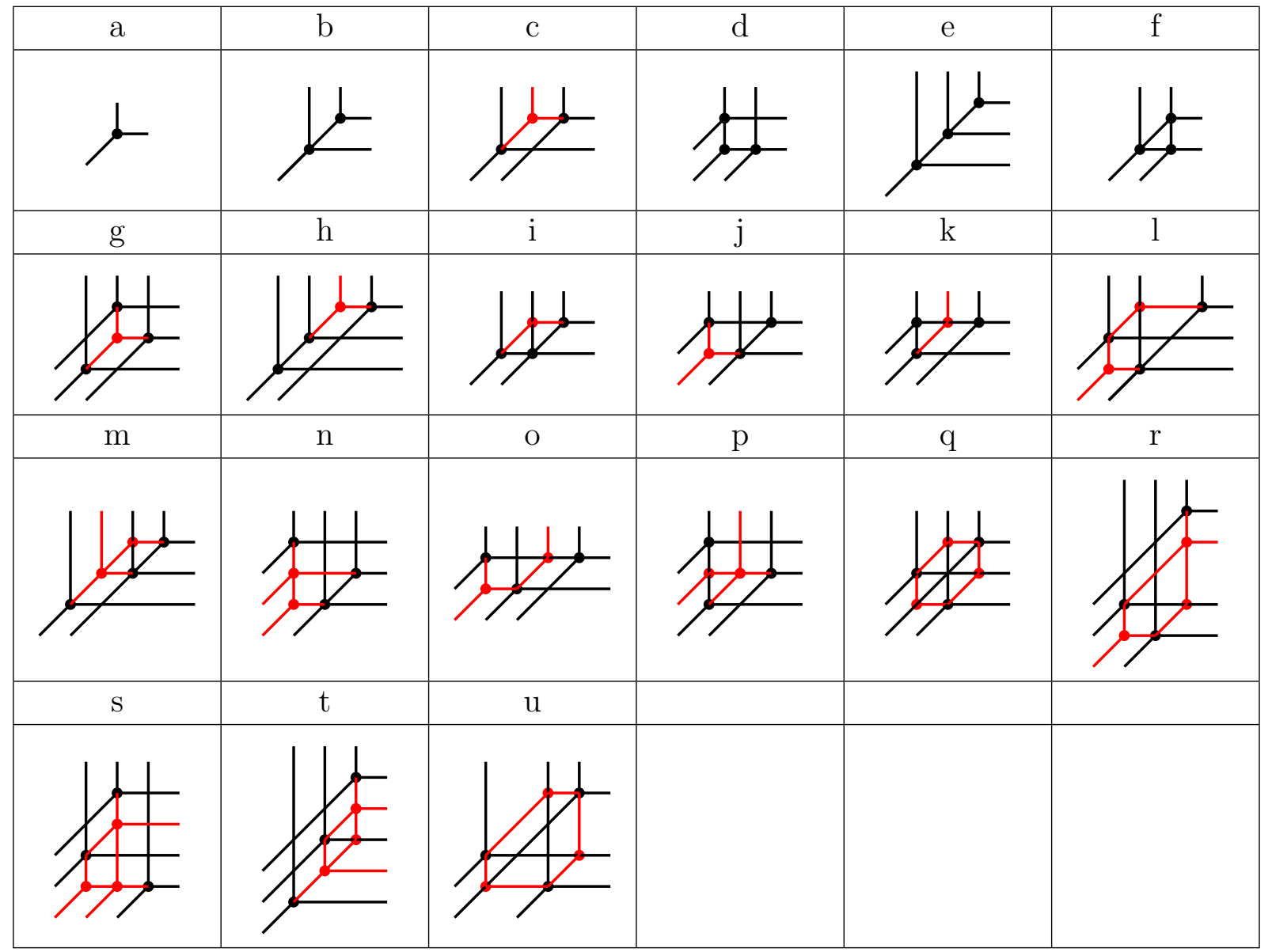

The next step is to define a refinement $\widetilde{\mathcal{R}}_{m}$ of the fan $\mathcal{R}_{m}$ of Definition 8.3 .

Lemma-Definition 9.8. Let $\widetilde{\mathcal{R}}_{m}$ be the collection of cones in $\mathbb{R}^{2 m}$ in the form

$$
\pi\left(Q^{-1}(\sigma) \cap \tau\right) \text { for } \sigma \in \mathcal{Q}_{m+N}^{\prime}, \tau \in \mathcal{P}^{m+N},
$$

where $Q: \mathbb{R}^{2 m+2 N} \rightarrow \mathbb{R}^{2 m+2 N} / \mathbb{R}^{2}$ is the quotient map. This collection is a fan and the quotient map $q: \mathbb{R}^{2 m} \rightarrow \mathbb{R}^{2 m} / \mathbb{R}^{2}$ induces a map of fans between $\widetilde{\mathcal{R}}_{m}$ and $\widetilde{\mathcal{Q}}_{m}$.

Proof. Take a cone $\eta=\pi\left(Q^{-1}(\sigma) \cap \tau\right) \in \widetilde{\mathcal{R}}_{m}$. Then $\left.\pi\right|_{\eta}$ is bijective with the inverse $\left.\iota\right|_{\pi(\eta)}$. Since cones of the form $Q^{-1}(\sigma) \cap \tau$ form a fan, it follows that $\widetilde{\mathcal{R}}_{m}$ is a fan as well. Next,

$$
q(\eta)=q\left(\pi\left(Q^{-1}(\sigma) \cap \tau\right)\right)=\bar{\pi}\left(Q\left(Q^{-1}(\sigma) \cap \tau\right)\right)=\bar{\pi}(\sigma \cap Q(\tau)) \subseteq \bar{\pi}(\sigma),
$$


TABLE 6. "Stretching" the decompositions $\mathcal{S}(v)$ in Table 5 creates further combinatorial types of $\widetilde{\mathcal{S}}(v)$ producing further cones in $\widetilde{\mathcal{Q}}_{3}$.

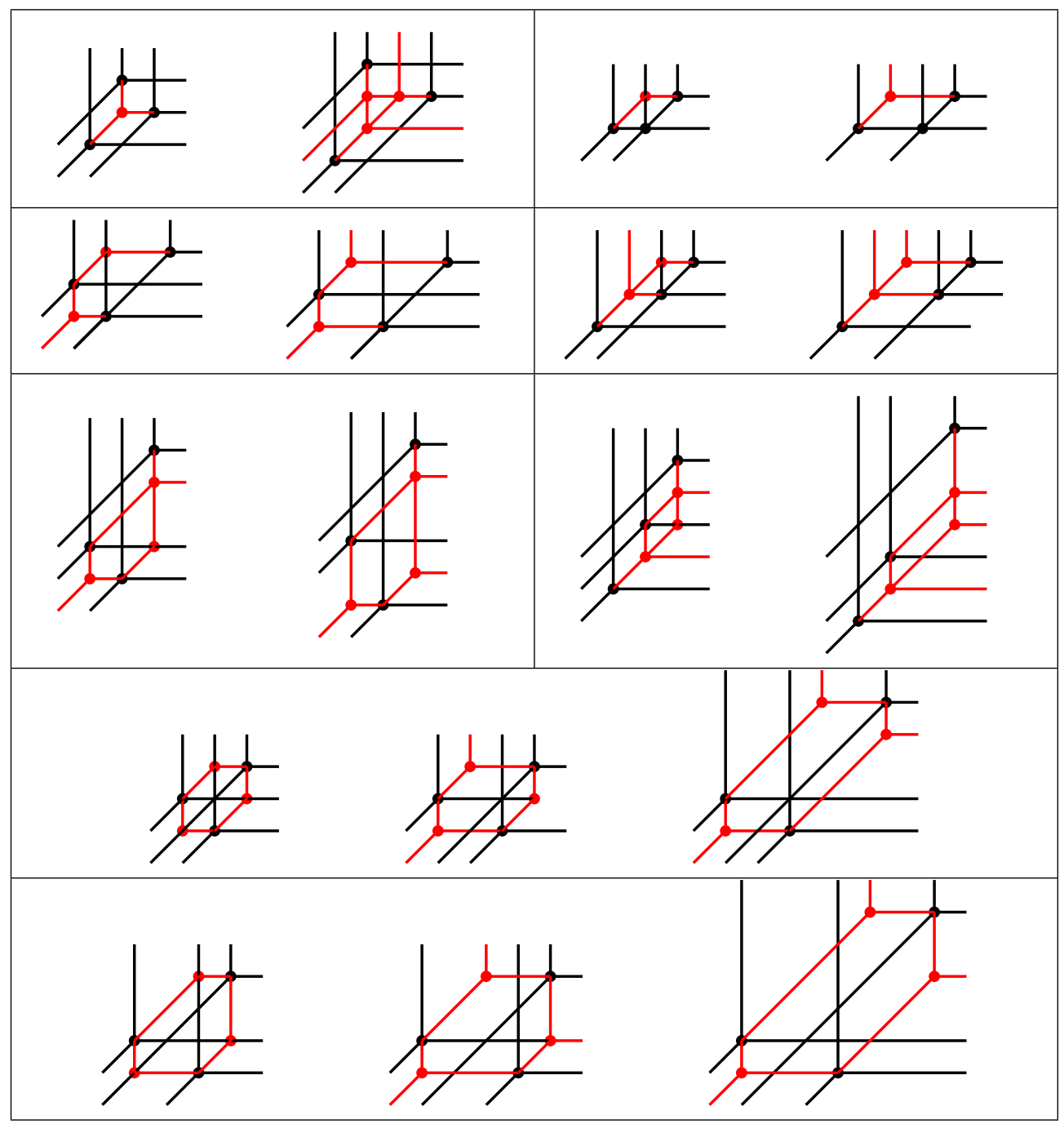

where $\bar{\pi}(\sigma) \in \widetilde{\mathcal{Q}}_{m}$ by definition. Hence $q$ induces a map of fans.

Lemma 9.9. Let $N=\left(\begin{array}{l}3 \\ 2\end{array}\right)\left(\begin{array}{c}m \\ 2\end{array}\right)$. We have a commutative diagram of toric morphisms

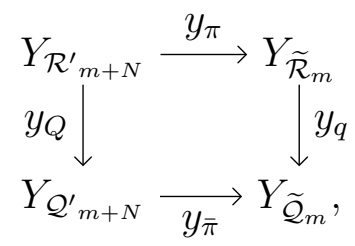

where $y_{\pi}$ and $y_{\bar{\pi}}$ are quotients by free $\mathbb{G}_{m}^{2 N}$-action. The morphism $y_{q}$ is flat with reduced fibers. 
Proof. The morphisms $y_{\pi}$ and $y_{\bar{\pi}}$ are induced by linear maps $\pi$ and $\bar{\pi}$ of fans which have piece-wise linear inverses $\iota$ and $\bar{\iota}$. This proves the first part because every affine toric chart of the variety on the left is isomorphic (not canonically) to the product of the corresponding affine toric chart of the variety on the right with $\mathbb{G}_{m}^{2 N}$. Since $y_{Q}$ is flat with reduced fibers, the same is true for $y_{q}$ by smooth descent.

Proposition 9.10. The family $Y_{\widetilde{\mathcal{R}}_{m}} \rightarrow Y_{\widetilde{\mathcal{Q}}_{m}}$ over the toric Gerrizen-Piwek space admits "light" sections $\ell_{1}, \ldots, \ell_{m}$ induced by linear maps $\lambda_{1}, \ldots, \lambda_{m}$ of Definition 8.8 and "heavy" constant sections $t_{1}, t_{2}, t_{3}$ defined as in Proposition 8.9.

Proof. The proof is the same as for Proposition 8.9.

\section{Projective duality and the Quotient fans $\mathcal{Q}_{m}$ And $\mathcal{Q}_{m}^{\vee}$}

In the construction of the quotient fan $\mathcal{Q}_{m}$ and its interpretation in terms of subdivisions of $\mathbb{R}^{2}$, we could have used the fan $\mathcal{P}^{\vee}=-\mathcal{P}$ of the dual projective plane $\left(\mathbb{P}^{2}\right)^{\vee}$ in place of $\mathcal{P}$. As in $\$ 8$, we define the dual quotient fan $\mathcal{Q}_{m}^{\vee}$. So we have the isomorphism

$$
\left(\left(\mathbb{P}^{2}\right)^{\vee}\right)^{m} / / H \cong Y_{\mathcal{Q}_{m}^{\vee}}
$$

where $H \subseteq \mathbb{P}^{2}$ is the dense torus. The cones in $\mathcal{Q}_{m}^{\vee}$ can be interpreted combinatorially as in Lemma 8.2 in terms of polyhedral subdivisions $\mathcal{S}(v)^{\vee}$ of $\mathbb{R}^{2}$ induced by dual spiders $S\left(v_{i}, v_{i}^{\prime}\right)^{\vee}$ for $i=1, \ldots, m$ (see Definition 9.1). Notice that the homomorphism $\mathbb{R}^{2 m} / \mathbb{R}^{2}$ sending $[v]$ to $-[v]$ maps the cones in $\mathcal{Q}_{m}$ to cones in $\mathcal{Q}_{m}^{\vee}$ (for an explicit example, see Figure 17). This induces a natural toric isomorphism between the Chow quotients $\left(\left(\mathbb{P}^{2}\right)^{\vee}\right)^{m} / / H$ and $\left(\mathbb{P}^{2}\right)^{m} / / H$.
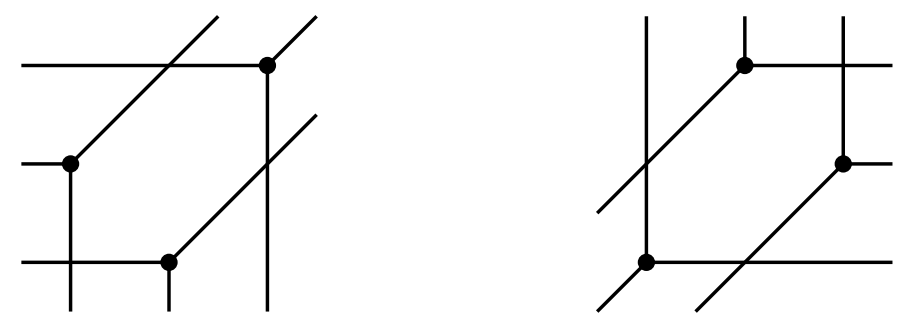

Figure 17 . On the left, subdivision of $\mathbb{R}^{2}$ given by a cone in $\mathcal{Q}_{3}^{\vee}$, and on the right its image under the homomorphism $[v] \mapsto-[v]$ given by a cone in $\mathcal{Q}_{3}$.

A surprising fact is that the fan $\widetilde{\mathcal{Q}}_{m}$ refines not only $\mathcal{Q}_{m}$ (Proposition 9.7) but also $\mathcal{Q}_{m}^{\vee}$. Before we can prove this fact in Theorem 10.5, we need a little preparation.

Definition 10.1. Given $[v] \in \mathbb{R}^{2 m} / \mathbb{R}^{2}$, for all distinct $i, j \in[m]$, let $\delta_{i j} \in \Sigma\left(v_{i}, v_{i}^{\prime}\right)$ be the minimal cone that contains $\left(-v_{j},-v_{j}^{\prime}\right)$. We call the collection of cones $\left\{\delta_{i j}\right\}_{1 \leq i, j \leq m, i \neq j}$ the cone data of $v$. If $\left\{\delta_{i j}\right\}$ and $\left\{\eta_{i j}\right\}$ are the cone data attached to two subdivisions $\mathcal{S}(v), \mathcal{S}(w)$, then we say that $\left\{\delta_{i j}\right\}$ and $\left\{\eta_{i j}\right\}$ are equivalent provided for all distinct $i, j \in[\mathrm{m}]$,

$$
\delta_{i j}+\left(v_{i}, v_{i}^{\prime}\right)=\eta_{i j}+\left(w_{i}, w_{i}^{\prime}\right) .
$$


Lemma 10.2. Let $[v],[w] \in \mathbb{R}^{2 m} / \mathbb{R}^{2}$ and consider the respective cone data $\left\{\delta_{i j}\right\}$ and $\left\{\eta_{i j}\right\}$. If $\mathcal{S}(v)$ and $\mathcal{S}(w)$ are combinatorially equivalent, then $\left\{\delta_{i j}\right\}$ and $\left\{\eta_{i j}\right\}$ are equivalent.

Proof. Assume that $\mathcal{S}(v)$ and $\mathcal{S}(w)$ are combinatorially equivalent. By Lemma 8.6, the subdivisions obtained by dropping all the spiders other than the $i$-th and the $j$-th, are also equivalent. This means precisely that the cone data are equivalent.

The next proposition analyzes the converse of the the previous lemma when $m=3$.

Proposition 10.3. Let $[v],[w] \in \mathbb{R}^{6} / \mathbb{R}^{2}$ and suppose their cone data $\left\{\delta_{i j}\right\}$ and $\left\{\eta_{i j}\right\}$ are equivalent. Then $\mathcal{S}(v)$ is combinatorially equivalent to $\mathcal{S}(w)$ unless they are combinatorially equivalent to (q) and (u) in Table 3, respectively (or vice versa). The subdivisions (q) and (u) have equivalent cone data but are not combinatorially equivalent.

Proof. Going through all the cases in Table 3, which we reproduced from CHSW11, Figure 6], shows that the combinatorial equivalence class of the plane subdivision is uniquely determined by the cone data except for the subdivisions $(\mathrm{q})$ and $(\mathrm{u})$.

Using the fans $\Sigma\left(v_{i}, v_{i}^{\prime}\right)^{\vee}$ instead of $\Sigma\left(v_{i}, v_{i}^{\prime}\right)$, we can associate the dual cone data $\left\{\delta_{i j}^{\vee}\right\}$. Analogous results for $\mathcal{S}(v)^{\vee}$ and $\left\{\delta_{i j}^{\vee}\right\}$ hold. We notice the following property.

Lemma 10.4. Let $[v],[w] \in \mathbb{R}^{2 m} / \mathbb{R}^{2}$. If $\widetilde{\mathcal{S}}(v)$ is combinatorially equivalent to $\widetilde{\mathcal{S}}(w)$, then the dual cone data $\left\{\delta_{i j}^{\vee}\right\}$ and $\left\{\eta_{i j}^{\vee}\right\}$ are equivalent.

Proof. Given a pair of distinct indices, we want to show that

$$
\delta_{i j}^{\vee}+\left(v_{i}, v_{i}^{\prime}\right)=\eta_{i j}^{\vee}+\left(w_{i}, w_{i}^{\prime}\right)
$$

Let $\delta_{i j} \in \Sigma\left(v_{i}, v_{i}^{\prime}\right)$ be the minimal cone containing $\left(-v_{j},-v_{j}^{\prime}\right)$. If $\delta_{i j}$ has dimension at most 1 , then we can immediately determine $\delta_{i j}^{\vee} \in \Sigma\left(v_{i}, v_{i}^{\prime}\right)^{\vee}$, and in each case it holds that $\delta_{i j}^{\vee}+$ $\left(v_{i}, v_{i}^{\prime}\right)=\eta_{i j}^{\vee}+\left(w_{i}, w_{i}^{\prime}\right)$. Therefore, assume that $\delta_{i j}$ is 2-dimensional, and without loss of generality assume that $\delta_{i j}$ is the cone whose two rays form a right angle (an analogous argument applies to the other 2-dimensional cones). Then $\delta_{i j}^{\vee}$ can be $\alpha, \beta$ or $\alpha \cap \beta$ in Figure 18 , and this is uniquely determined by the position of $\left(-v_{j},-v_{j}^{\prime}\right)$ with respect to $\left(-x_{i j},-x_{i j}\right)$

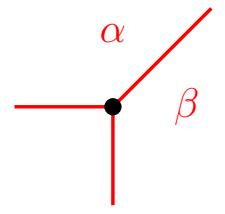

Figure 18. The fan $\Sigma\left(v_{i}, v_{i}^{\prime}\right)^{\vee}$ and two of its maximal cones.

(see Definition 9.3). We have three possible cases, which are pictured in Figure 19:

(1) $\left(x_{i j}, x_{i j}^{\prime}\right)=\left(v_{j}, v_{j}^{\prime}\right)$ if and only if $\left(-v_{j},-v_{j}^{\prime}\right)$ is contained in the relative interior of a ray in $\Sigma\left(v_{i}, v_{i}^{\prime}\right)^{\vee}$. If this is the case, then we have that $\delta_{i j}=\alpha \cap \beta$.

(2) If $-x_{i j}=-v_{j}$ and $-x_{i j}^{\prime}<-v_{j}^{\prime}$, then $\delta_{i j}=\alpha$.

(3) Lastly, if $-x_{i j}<-v_{j}$ and $-x_{i j}^{\prime}=-v_{j}^{\prime}$, then $\delta_{i j}=\beta$. 

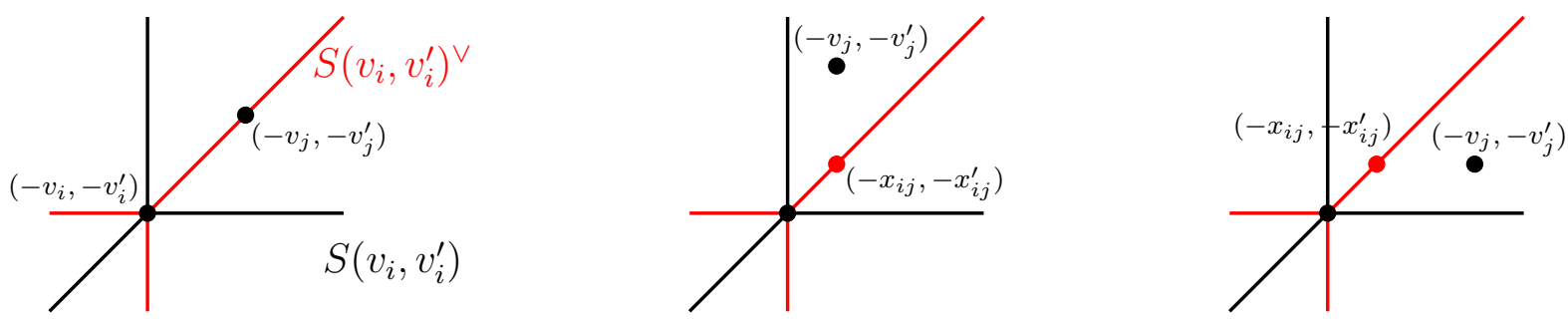

Figure 19. Possible positions of $\left(-v_{j},-v_{j}^{\prime}\right)$.

In each case, $\delta_{i j}^{\vee}+\left(v_{i}, v_{i}^{\prime}\right)=\eta_{i j}^{\vee}+\left(w_{i}, w_{i}^{\prime}\right)$.

Theorem 10.5. The fan $\widetilde{\mathcal{Q}}_{m}$ refines the dual quotient fan $\mathcal{Q}_{m}^{\vee}$.

Proof. Let $[v],[w] \in \mathbb{R}^{2 m} / \mathbb{R}^{2}$ be two vectors lying in the relative interior of the same cone of $\widetilde{\mathcal{Q}}_{m}$, which means that the polyhedral subdivisions $\widetilde{\mathcal{S}}(v)$ and $\widetilde{\mathcal{S}}(w)$ are combinatorially equivalent. We want to show that $\mathcal{S}(v)^{\vee}$ and $\mathcal{S}(w)^{\vee}$ are combinatorially equivalent as well.

If $m=1$ then this is true because $\mathcal{S}(v)^{\vee}$ and $\mathcal{S}(w)^{\vee}$ are equal up to a translation in $\mathbb{R}^{2}$. If $m=2$ then the combinatorial equivalence class $[\widetilde{\mathcal{S}}(v)]$ uniquely determines $[\mathcal{S}(v)]$, which uniquely determines $\left[\mathcal{S}(v)^{\vee}\right]$. So if $[\widetilde{\mathcal{S}}(v)]=[\widetilde{\mathcal{S}}(w)]$, then $\left[\mathcal{S}(v)^{\vee}\right]=\left[\mathcal{S}(w)^{\vee}\right]$.

For $m \geq 3$ we argue by induction. Let $m=3$. By Lemma 10.4, the dual cone data $\left\{\delta_{i j}^{\vee}\right\},\left\{\eta_{i j}^{\vee}\right\}$ associated to $[v],[w]$ respectively are equivalent. Therefore, by Proposition 10.3 adapted to the dual case, $\mathcal{S}(v)^{\vee}$ is equivalent to $\mathcal{S}(w)^{\vee}$ provided we are away from the exceptional case in the first row of Table 7. However, this case can not occur: $\widetilde{\mathcal{S}}(v)$ and $\widetilde{\mathcal{S}}(w)$ are not combinatorially equivalent as we can see in the second row of Table 7 .

TABLE 7. Exceptional case for $m=3$ in the proof of Theorem 10.5 .

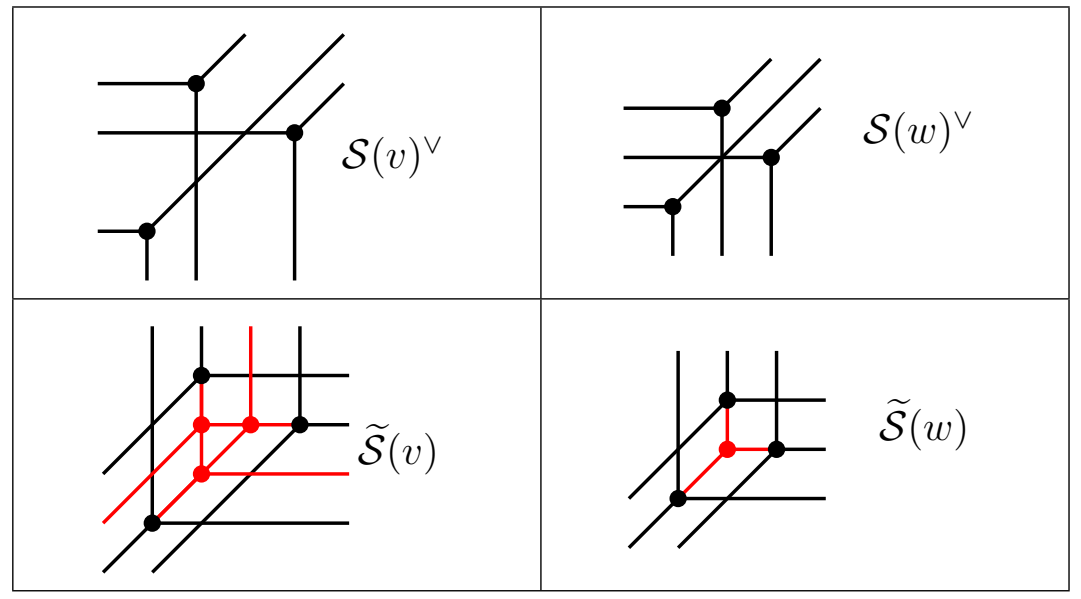

Now assume the conclusion for $m-1 \geq 3$ and let us prove it for $m$. Consider cones $\sigma_{i}^{\vee} \in \Sigma\left(v_{i}, v_{i}^{\prime}\right)^{\vee}, \tau_{i}^{\vee} \in \Sigma\left(w_{i}, w_{i}^{\prime}\right)^{\vee}$ such that $\sigma_{i}^{\vee}+\left(v_{i}, v_{i}^{\prime}\right)=\tau_{i}^{\vee}+\left(w_{i}, w_{i}^{\prime}\right)$ and assume that $\cap_{i=1}^{m} \sigma_{i}^{\vee} \neq \emptyset$. We want to show that $\cap_{i=1}^{m} \tau_{i}^{\vee}$ is also nonempty, which, by Helly's theorem, 
is equivalent to $\tau_{i_{1}}^{\vee} \cap \tau_{i_{2}}^{\vee} \cap \tau_{i_{3}}^{\vee} \neq \emptyset$ for every choice of distinct indices $i_{1}, i_{2}, i_{3} \in[m]$. Since $m>3$, it will be enough to show that $\cap_{i \neq j} \tau_{i}^{\vee} \neq \emptyset$ for each choice of index $j \in[m]$. So consider the projection map $p_{j}: \mathbb{R}^{2 m} \rightarrow \mathbb{R}^{2 m-2}$. By Proposition 9.7 we have that $\widetilde{\mathcal{S}}\left(p_{j}(v)\right)$ is combinatorially equivalent to $\widetilde{\mathcal{S}}\left(p_{j}(w)\right)$. So by the inductive assumption we have that $\mathcal{S}^{\vee}\left(p_{j}(v)\right)$ is combinatorially equivalent to $\mathcal{S}\left(p_{j}(w)\right)^{\vee}$. This means that $\cap_{i \neq j} \tau_{i}^{\vee}$ is nonempty because $\emptyset \neq \cap_{i=1}^{m} \sigma_{i}^{\vee} \subseteq \cap_{i \neq j} \sigma_{i}^{\vee}$, concluding the proof.

\section{Planar locus in $\overline{\mathbf{X}}(3, n)$ and $\overline{\mathbf{X}}_{\mathrm{GP}}(3, n)$}

Given rational weights $\mathbf{b}=\left(b_{1}, \ldots, b_{n}\right), 0<b_{i} \leq 1$, satisfying $\sum_{i=1}^{n} b_{i}>3$, by [Ale15] there exists a projective moduli space $\overline{\mathbf{M}}_{\mathbf{b}}(3, n)$ parametrizing stable pairs $\left(\mathbb{P}^{2}, \sum_{i=1}^{n} b_{i} L_{i}\right)$, where $L_{i} \subseteq \mathbb{P}^{2}$ are lines, and their stable degenerations. For $\mathbf{b}=(1, \ldots, 1), \overline{\mathbf{M}}_{\mathbf{b}}(3, n)=\overline{\mathbf{X}}(3, n)$. On the other hand, choose weights such that three of them, say $b_{i}, b_{j}, b_{k}$, are equal to 1 and the remaining weights are equal to $\epsilon \ll 1$. By [Ale08, Example 9.6], we have an isomorphism $\overline{\mathbf{M}}_{\mathbf{b}}(3, n) \cong\left(\left(\mathbb{P}^{2}\right)^{\vee}\right)^{n-3} / / H$, and so its normalization is isomorphic to $Y_{\mathcal{Q}_{n-3}^{\vee}}$. Setting $m=n-3$, we obtain a commutative diagram of birational maps and morphisms:

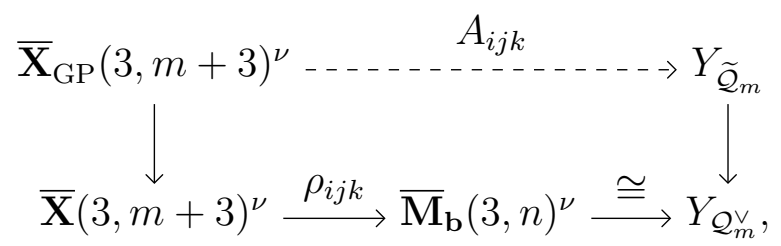

where the morphism $Y_{\widetilde{\mathcal{Q}}_{m}} \rightarrow Y_{\mathcal{Q}_{m}^{\vee}}$ exists by Theorem 10.5. In what follows we focus on the case $(i, j, k)=(1,2,3)$. The same results hold for other cases after permuting the labels. The commutative diagram is a diagram of isomorphisms over $\mathbf{B}_{m+3} \cong \mathbf{X}(3, n)$.

Definition 11.1. Let $P=\Delta(3, m+3)$ and consider Lafforgue's toric variety $\mathcal{A}^{P}$. Let $\mathcal{A}_{123}^{P}$ the union of the toric strata of $\mathcal{A}^{P}$ corresponding to regular matroid polytope subdivisions of $P$ whose maximal dimensional polytopes contain the vertex $e_{1}+e_{2}+e_{3}$, where $e_{1}, \ldots, e_{m+3}$ is the canonical basis of $\mathbb{R}^{m+3}$. Notice that $\mathcal{A}_{123}^{P}$ is an open subset because if a polyhedral subdivision has a maximal polytope not containing $e_{1}+e_{2}+e_{3}$, then any refinement of it does, so the complement is closed. This induces an open subset

$$
\mathbf{U}^{123} \subseteq \overline{\mathbf{X}}(3, m+3)^{\nu}
$$

and its preimage $\mathbf{U}_{\mathrm{GP}}^{123} \subseteq \overline{\mathbf{X}}_{\mathrm{GP}}(3, m+3)^{\nu}$. We have the following commutative diagram:

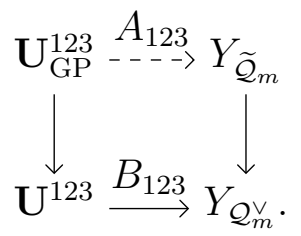

The planar locus $\mathbf{U}(3, n) \subseteq \overline{\mathbf{X}}(3, n)^{\nu}$ (resp. $\left.\mathbf{U}_{\mathrm{GP}}(3, n) \subseteq \overline{\mathbf{X}}_{\mathrm{GP}}(3, n)^{\nu}\right)$ is the union of $\mathbf{U}^{i j k}$ (resp. $\left.\mathbf{U}_{\mathrm{GP}}^{i j k}\right)$ for all possible triples $i, j, k$. 
Theorem 11.2. The map $A_{123}$ is regular and both maps $A_{123}$ and $B_{123}$ are open embeddings. The planar loci $\mathbf{U}(3, n) \subseteq \overline{\mathbf{X}}(3, n)^{\nu}$ and $\mathbf{U}_{\mathrm{GP}}(3, n) \subseteq \overline{\mathbf{X}}_{\mathrm{GP}}(3, n)^{\nu}$ have toroidal singularities.

Proof. We start with $B_{123}$. Let $\left(\mathcal{X}, \sum_{i=1}^{m+3} \mathcal{L}_{i}\right)$ be the family of stable pairs over $\overline{\mathbf{X}}(3, m+3)$ and take its fiber $\left(X, \sum_{i=1}^{m+3} L_{i}\right)$ over a point in $\mathbf{U}^{123}$. By [KT06, $\left.\S 8\right], X$ is a stable toric surface with the stable toric boundary given by $L_{1}+L_{2}+L_{3}$. In particular, $K_{X}+L_{1}+L_{2}+L_{3} \sim 0$. Concretely, we can realize $\left(X, \sum_{i=1}^{m+3} L_{i}\right)$ as the central fiber of the pullback of the family of stable pairs to $\operatorname{Spec} R$ via some arc $\overline{\mathbf{a}}: \operatorname{Spec} R \rightarrow \overline{\mathbf{X}}(3, m+3)$ such that $\overline{\mathbf{a}}(\operatorname{Spec} K) \in$ $\mathbf{X}(3, m+3)(K)$. Since all maximal-dimensional polytopes in the matroid decomposition share a vertex $e_{1}+e_{2}+e_{3}$, all stable lattices for the arc are contained in the corresponding apartment [KT06, §8], and therefore $X$ is a stable toric surface that corresponds to a regular mixed polyhedral subdivision of the dual simplex $m \Delta_{2}^{\vee}$. The broken lines $L_{1}, \ldots, L_{n}$ intersect pairwise at smooth points of $X$ [HKT06, Theorem 1.1].

It follows that the pair $\left(X, \sum_{i=1}^{3} L_{i}+\sum_{i=4}^{m+3} \epsilon L_{i}\right)$ is semi-log canonical and the $\mathbb{Q}$-line bundle

$$
K_{X}+\sum_{i=1}^{3} L_{i}+\sum_{i=4}^{m+3} \epsilon L_{i} \sim \epsilon \cdot \sum_{i=4}^{m+3} L_{i}
$$

is ample because $K_{X}+\sum_{i=1}^{m+3} L_{i} \sim \sum_{i=4}^{m+3} L_{i}$ is ample. Therefore, the restriction of the family $\left(\mathcal{X}, \sum_{i=1}^{m+3} \mathcal{L}_{i}\right)$ to $\mathbf{U}^{123}$ embeds the latter in $\overline{\mathbf{M}}_{\mathbf{b}}(3, n)$ as an open subset.

Next, we consider $A_{123}$. Recall that $\overline{\mathbf{X}}_{\mathrm{GP}}(3, n)$ is defined as the closure of $\mathbf{B}_{n}$ in the multigraded Hilbert scheme of $\left(\mathbb{P}^{2}\right)\left(\begin{array}{c}n \\ 4\end{array}\right)$. Write $n=3+m, N=\left(\begin{array}{l}3 \\ 2\end{array}\right)\left(\begin{array}{c}m \\ 2\end{array}\right)$ and $m+N+M=\left(\begin{array}{c}3+m \\ 4\end{array}\right)$.

The pullback to $\overline{\mathbf{X}}_{\mathrm{GP}}(3, n)$ of the universal family of the (ordinary) Hilbert scheme is a flat family $\overline{\mathcal{M}}$ with $n$ sections. For any point $x \in \overline{\mathbf{X}}_{\mathrm{GP}}(3, n)$, the fiber $\overline{\mathcal{M}}_{x}$ is a reduced surface $\left(X ; p_{1}, \ldots, p_{n}\right)$ with $n$ smooth marked points. For every quadruple $I=\left\{i_{1}, \ldots, i_{4}\right\} \subseteq[n]$, the morphism $X \rightarrow \mathbb{P}^{2}$ induced by the projection onto the $I$-th component of $\left(\mathbb{P}^{2}\right)^{\left(\begin{array}{l}n \\ 4\end{array}\right)}$ sends $p_{i_{1}}, \ldots, p_{i_{4}}$ to the points $[1: 0: 0],[0: 1: 0],[0: 0: 1]$, and $[1: 1: 1]$. The surface $\left(X ; p_{1}, \ldots, p_{n}\right)$ is the special fiber of the Mustafin join $\mathbb{P}\left(\Sigma_{\mathbf{a}}\right)$ for any arc $\mathbf{a}:$ Spec $K \rightarrow \mathbf{B}_{n}$ with a given limit point $\overline{\mathbf{a}}(0)=x \in \overline{\mathbf{X}}_{\mathrm{GP}}(3, n)$.

The open subset $\mathbf{U}_{\mathrm{GP}}^{123}$ parametrizes surfaces such that $\Sigma_{\mathbf{a}}$ is contained in the apartment that corresponds to $a_{1}, a_{2}, a_{3}$ (for any arc converging to $x$ ).

Lemma 11.3. The restriction of the projection $\left(\mathbb{P}^{2}\right)^{m+N+M} \rightarrow\left(\mathbb{P}^{2}\right)^{m+N}$ to every marked surface $\left(X ; p_{1}, \ldots, p_{n}\right)$ parametrized by $\mathbf{U}_{\mathrm{GP}}^{123}$ is an isomorphism. Moreover, the restriction to $\mathrm{U}_{\mathrm{GP}}^{123}$ of the induced morphism from the multigraded Hilbert scheme

$$
\mathbf{H}\left(\left(\mathbb{P}^{2}\right)^{m+N+M}\right) \rightarrow \operatorname{Hilb}\left(\left(\mathbb{P}^{2}\right)^{m+N}\right)
$$


to the ordinary Hilbert scheme is bijective onto its image, let $\mathbf{V}$ be its normalization.

Proof. To prove the first statement, we choose an arc a and represent $\left(X ; p_{1}, \ldots, p_{n}\right)$ as the special fiber of the corresponding Mustafin join. Since all stable lattices are contained in the apartment that corresponds to sections $a_{1}, a_{2}, a_{3}$, the set $\Sigma_{\mathbf{a}}$ is the set of lattices $L_{i}$, $L_{\alpha \beta, i j}$ in the notation of Lemma 9.2 . Thus $X$ is isomorphic to a subscheme in $\left(\mathbb{P}^{2}\right)^{m+N}$ via projection. To prove the second statement, it suffices to prove that the morphisms $X \rightarrow \mathbb{P}_{I}^{2}$ parametrized by quadruples $I$ indexed by $M$ are uniquely determined by the morphisms $X \rightarrow \mathbb{P}_{J}^{2}$ parametrized by quadruples $J$ indexed by $m+N$. Since $\Sigma_{\mathbf{a}}$ is contained in the apartment that corresponds to the sections $a_{1}, a_{2}, a_{3}$, for every quadruple $I=\left\{i_{1}, \ldots, i_{4}\right\}$ indexed by $M$, there exists a quadruple $J$ indexed by $m+N$ such that the image of the quadruple $p_{i_{1}}, \ldots, p_{i_{4}}$ in $\mathbb{P}_{J}^{2}$ is in linearly general position. Thus the morphism $X \rightarrow \mathbb{P}_{I}^{2}$ is a composition of the morphism $X \rightarrow \mathbb{P}_{J}^{2}$ and an isomorphism $\mathbb{P}_{J}^{2} \rightarrow \mathbb{P}_{I}^{2}$ uniquely determined by the marked surface $\left(X ; p_{1}, \ldots, p_{n}\right)$ and its morphism to $\mathbb{P}_{J}^{2}$.

At this point we can forget about $\mathbf{U}_{\mathrm{GP}}^{123}$ and work with $\mathbf{V}$ instead, which is a normalization of the partial compactification of $\mathbf{B}_{n}$ in the Hilbert scheme of $\left(\mathbb{P}^{2}\right)^{m+N}$ parametrizing limits such that all stable lattices are in the apartment that corresponds to the first three sections (for some choice of an arc). The pullback $\mathcal{X} \rightarrow \mathbf{V}$ of the universal family of the Hilbert scheme is smooth along $n$ disjoint sections giving the same fibers $\left(X ; p_{1}, \ldots, p_{n}\right)$ as before. Composing sections with projections gives morphisms

$$
\mathcal{P}_{1}^{I}, \ldots, \mathcal{P}_{n}^{I}: \mathbf{V} \rightarrow \mathbb{P}^{2}
$$

for every quadruple $I$ of the form $(1,2,3, i)$ (first type) or $(\alpha, \beta, i, j)$ (second type). Note that the points $\mathcal{P}_{1}^{I}(v), \mathcal{P}_{2}^{I}(v), \mathcal{P}_{3}^{I}(v)$ are linearly independent for any $v \in \mathbf{V}$. Moreover, for quadruples of the first type they are the standard points $e_{1}, e_{2}, e_{3}$. However, for quadruples of the second type only $\mathcal{P}_{\alpha}^{I}(v)$ and $\mathcal{P}_{\beta}^{I}(v)$ are standard points $e_{1}$ and $e_{2}$, since by definition (see the Introduction) $\mathcal{P}_{i}^{I}(v)=e_{3}$ (and $\mathcal{P}_{j}^{I}(v)=e_{4}=[1: 1: 1]$ ). For a fixed $v$ and a quadruple $I$ of the second type, we can find an automorphism of $\mathbb{P}^{2}$ that sends $e_{1}$ to $e_{\alpha}, e_{2}$ to $e_{\beta}$ and $\mathcal{P}_{\gamma}^{I}(v)$ to $e_{\gamma}$, where $\{\alpha, \beta, \gamma\}=\{1,2,3\}$. This automorphism is determined uniquely up to the $\mathbb{G}_{m}^{2}$-action. This gives a $\mathbb{G}_{m}^{2 N}$-torsor $\psi: \widetilde{\mathbf{V}} \rightarrow \mathbf{V}$ and an isomorphism

$$
\Psi: \widetilde{\mathbf{V}} \times\left(\mathbb{P}^{2}\right)^{m+N} \rightarrow \widetilde{\mathbf{V}} \times\left(\mathbb{P}^{2}\right)^{m+N},
$$

which is defined to be the identity on the first factor. Let $\widetilde{\mathcal{X}} \rightarrow \widetilde{\mathbf{V}}$ be the family $\Psi\left(\psi^{*} \mathcal{X}\right)$.

Since $\mathbf{B}_{n}$ is an open subset of an algebraic torus $\mathbb{G}_{m}^{2 m-2}$ and $\psi$ is a torsor, $\psi^{-1}\left(\mathbf{B}_{n}\right)$ is an open subset of an algebraic torus $\mathbb{G}_{m}^{2(m+N)-2}$. The morphism of $\psi^{-1}\left(\mathbf{B}_{n}\right)$ into the Hilbert scheme of $\left(\mathbb{P}^{2}\right)^{m+N}$ given by the family $\widetilde{\mathcal{X}}$ agrees with the embedding of $\mathbb{G}_{m}^{2(m+N)-2}$ into this Hilbert scheme as in $\$ 8$. Indeed, note that the first three sections of $\widetilde{\mathcal{X}}$ are constant sections given by the $\mathbb{G}_{m}^{2}$-invariant points in $\mathbb{P}^{2}$, thus every fiber of $\widetilde{\mathcal{X}}$ over $\psi^{-1}\left(\mathbf{B}_{n}\right)$ is a $\mathbb{P}^{2}$ embedded into $\left(\mathbb{P}^{2}\right)^{m+N}$ by torus translates. Therefore, $\tilde{\mathbf{V}}$ is a partial compactification of $\psi^{-1}\left(\mathbf{B}_{n}\right)$ in the toric Kapranov space $Y_{\mathcal{Q}_{m+N}}$. By Lemma 9.2 and Lemma-Definition 9.5, $\widetilde{\mathbf{V}}$ is an open subset of $Y_{\mathcal{Q}_{m+N}^{\prime}}$ and therefore, as claimed, $\mathbf{V}=\mathbf{V} / \mathbb{G}_{m}^{2 N}$ is isomorphic to an open subset of $Y_{\mathcal{Q}_{m+N}^{\prime}} / \mathbb{G}_{m}^{2 N}=Y_{\widetilde{\mathcal{Q}}_{m}}$ by Lemma 9.9 . 


\section{Study of $\overline{\mathbf{X}}_{\mathrm{GP}}(3,6)$}

In this section we explicitly describe the compactification $\overline{\mathbf{X}}_{\mathrm{GP}}(3,6)$ using the theory developed in 8811 . We classify the 6 -pointed degenerations parametrized by $\overline{\mathbf{X}}_{\mathrm{GP}}(3,6)$, show their relation to the corresponding line arrangement degenerations parametrized by Kapranov's Chow quotient $\overline{\mathbf{X}}(3,6)$, and finally prove that $\overline{\mathbf{X}}_{\mathrm{GP}}(3,6)^{\nu}$ is a tropical compactification of $\mathbf{X}(3,6)$ given by a specific polyhedral subdivision of the tropical Grassmannian $\Sigma(3,6)$.

Proposition 12.1. Up to permuting the labels, the degenerations parametrized by the planar locus $\mathbf{U}_{\mathrm{GP}}(3,6)$ are listed in Tables 14, 15, and 16.

Proof. We first classify all cones in the quotient fan $\mathcal{Q}_{3}$, i.e. all possible decompositions $\mathcal{S}(v)$ for integral $v \in\left(\mathbb{R}^{2}\right)^{3}$. All the possibilities are computed in [CHSW11, Figure 6], and we list them in Table 3 . Then, we need to classify all cones in the fan $\widetilde{\mathcal{Q}}_{3}$, i.e. all possible subdivisions $\widetilde{\mathcal{S}}(v)$, which are listed in Tables 5 and 6 . From these, we can determine the corresponding central fibers $X$ of Mustafin joins by Remark 5.3 .

Next, we need to put markings $p_{1}, \ldots, p_{6}$ on $X$. First, we choose three labeled points $p_{i}, p_{j}, p_{k}$ and put them in the three vertices of the degeneration (we do not always choose just $p_{1}, p_{2}, p_{3}$ for the reason explained in Remark 12.2). These three points are in general linear position in all primary components. By Proposition 9.10, the remaining "light" points are arranged according to Proposition 8.10, so that the $i$-the point belongs to the component that corresponds to $\left(v_{i}, v_{i}^{\prime}\right)$. As a guide, case \#8 was already worked out in Example 7.14. The final result is depicted in Tables 14, 15, and 16 .

Remark 12.2. The numbering of the degenerations in Tables 14, 15, and 16 is determined as follows. Given $\mathbf{a} \in\left(\mathbb{P}^{2}\right)^{6}(K)$ in general linear position over $K$, we have $x=\overline{\mathbf{a}}(0) \in \overline{\mathbf{X}}_{\mathrm{GP}}(3,6)$. Interpreting a as a one-parameter family of lines in $\left(\mathbb{P}^{2}\right)^{\vee}$ instead, we obtain another limit $y \in \overline{\mathbf{X}}(3,6)$, and $x \mapsto y$ under the morphism $\overline{\mathbf{X}}_{\mathrm{GP}}(3,6) \rightarrow \overline{\mathbf{X}}(3,6)$. Recall that the boundary of Kapranov's compactification $\overline{\mathbf{X}}(3,6)$ is stratified according to the regular matroid polytope subdivisions of the hypersimplex $\Delta(3,6)$. These are listed up to $S_{6}$-action in Ale15, Table 4.4]. Each boundary stratum corresponds to degenerate KSBA stable pairs listed in [Ale15, Figures 5.11, 5.12, and 5.13], which we reproduce with permission of the author in Figures 23 . 24. and 25 (notice that the degeneration \#16 corresponds to the matroid subdivision no. 16 in [Ale15, Table 4.4] with (3) $x_{1234} \leq 2, x_{12} \leq 1$ instead).

We claim that a degeneration of six points in $\mathbb{P}^{2}$ labeled by $\# N$ in our tables is mapped to the interior of the Kapranov's stratum \#N. To establish this matching, let us first look at an example. Assume $y$ belongs to the interior of the Kapranov's stratum corresponding to the matroid polytope subdivision no. 8 in [Ale15, Table 4.4]. This gives rise to the degenerate line arrangements in $\mathbb{P}^{2}$ in the first row of Table 8 . The projective dual of these line degenerations are in the second row of the same table. Now, the marking of $\left(\mathbb{P}\left(\Sigma_{\mathbf{a}}\right)_{\mathbb{k}} ; \bar{a}_{1}(0), \ldots, \bar{a}_{6}(0)\right)$ parametrized by $x$ must be such that on the primary components the image of the markings are given in the second row of Table 8. Such degeneration is given in Figure 12. One can inspect case by case that the degenerations listed in Tables 14 and 15 match bijectively the matroid subdivisions in [Ale15, Table 4.4]. We deal with the Kapranov's stratum \#7 
TABLE 8. In the first row, degenerate line arrangements corresponding to three matroid polytopes in subdivision no. 8 in [Ale15, Table 4.4]. In the second row, their respective projectively dual arrangements from Figure 12 .

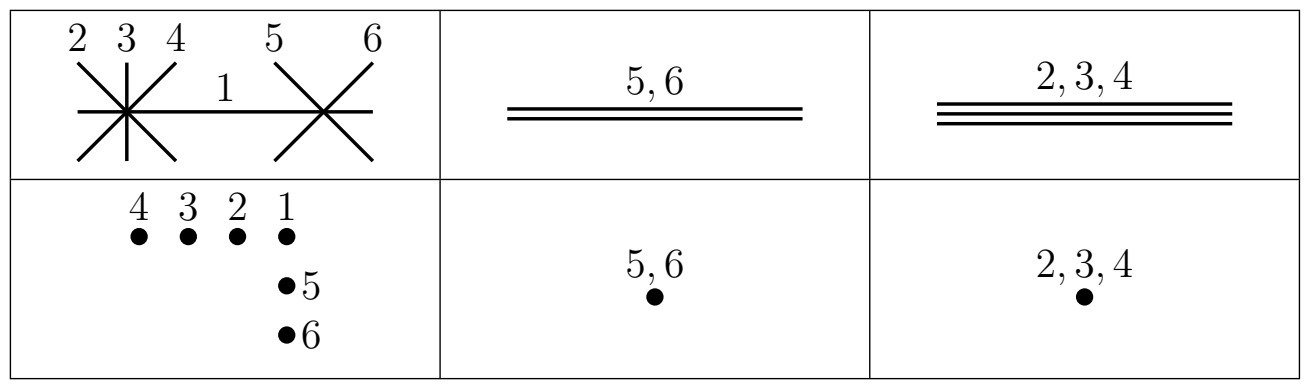

separately in Lemma 12.5. Lastly, in Table 16, the numbering \#12.1, \#15.1, and so on correspond to the stretches of spiders in Table 6 .

Next we describe the boundary stratification of $\overline{\mathbf{X}}(3,6)$ and construct its explicit blow up $\widehat{\mathbf{X}}(3,6)$ based on the analysis of the toroidal morphism of planar loci $\mathbf{U}_{\mathrm{GP}}(3,6) \rightarrow \mathbf{U}(3,6)$. Then we will add an ad hoc analysis of the non-planar locus to prove $\widehat{\mathbf{X}}(3,6) \cong \overline{\mathbf{X}}_{\mathrm{GP}}(3,6)^{\nu}$. The space $\mathbf{X}(3,6)=G^{0}(3,6) / \mathbb{G}_{m}^{5}$ is a very affine variety, i.e. a closed subset of the torus

$$
T=\mathbb{G}_{m}^{\left(\begin{array}{c}
6 \\
3
\end{array}\right)-1} / \mathbb{G}_{m}^{5}
$$

It was proved in [Lux08] that Kapranov's compactification $\overline{\mathbf{X}}(3,6)$ can be obtained as the Zariski closure of $\mathbf{X}(3,6)$ inside the toric variety $Y_{\Sigma(3,6)}$ with dense open subtorus $T$, where the fan $\Sigma(3,6)$ is the tropical Grassmannian of Speyer-Sturmfels in SS04. Moreover, $\overline{\mathbf{X}}(3,6)$ is tropical, which means that $\overline{\mathbf{X}}(3,6)$ is proper and the multiplication map

$$
T \times \overline{\mathbf{X}}(3,6) \rightarrow Y_{\Sigma(3,6)}
$$

is faithfully flat ([Tev07, Definition 1.1]). Even better, Luxton proves in [Lux08] that the multiplication map is smooth (and so $\mathbf{X}(3,6)$ is schön [Tev07, Definition 1.3]). Adopting the notation in [Lux08], the fan $\Sigma(3,6)$ has three types of rays, which correspond to boundary divisors of $\overline{\mathbf{X}}(3,6)$. In parentheses, we give the corresponding notation from [SS04].

(1) For each triple of distinct indices $i, j, k \in[6]$, we have a ray ( $i j k$ ) (rays of type E).

(2) For each pair of distinct indices $i, j \in[6]$, we have a ray $(i j)$ (rays of type $\mathrm{F}$ ).

(3) For each partition $\{\{i, j\},\{k, \ell\},\{m, n\}\}$ of [6], we have two distinct rays denoted by $(i j)(k \ell)(m n)$ and $(i j)(m n)(k \ell)$ (these are the rays of type $\mathrm{G}$ in [SS04]).

There is a bijective correspondence between the boundary strata of $\overline{\mathbf{X}}(3,6)$ and the toric strata of $Y_{\Sigma(3,6)}$, i.e. the cones of $\Sigma(3,6)$. Explicitly, up to $S_{6}$-action, consider the labeling of the strata of $\overline{\mathbf{X}}(3,6)$ given in [Ale15. Then this correspondence is given in Table 9 .

Note that only the cone \#20 (of type FFFGG) is not simplicial. These cones are known as the bipyramid cones: each one can be obtained by taking the cone over an appropriate triangular bipyramid, which is the gluing of two tetrahedra along a common facet. 
TABle 9. Correspondence between strata of $\overline{\mathbf{X}}(3,6)$ and cones of $\Sigma(3,6)$.

\begin{tabular}{|l|c||c|c|}
\hline$\# 1$ & $(123)$ & $\# 14$ & $(12)(34)(56),(356)$ \\
\hline$\# 2$ & $(123),(456)$ & $\# 15$ & $(12)(34)(56),(12),(56)$ \\
\hline$\# 3$ & $(124),(456)$ & $\# 16$ & $(12),(56),(124)$ \\
\hline$\# 4$ & $(456),(124),(135)$ & $\# 17$ & $(12)(34)(56),(125),(12)$ \\
\hline$\# 5$ & $(56)$ & $\# 18$ & $(12)(34)(56),(356),(12)$ \\
\hline$\# 6$ & $(56),(123)$ & $\# 19$ & $(12)(34)(56),(125),(356)$ \\
\hline$\# 7$ & $(123),(145),(246),(356)$ & $\# 20$ & $(12)(34)(56),(12)(56)(34),(12),(34),(56)$ \\
\hline$\# 8$ & $(56),(156)$ & $\# 21$ & $(12)(34)(56),(34),(56),(256)$ \\
\hline$\# 9$ & $(123),(56),(156)$ & $\# 22$ & $(12)(34)(56),(34),(256),(346)$ \\
\hline$\# 10$ & $(234),(56),(156)$ & $\# 23$ & $(34),(56),(134),(256)$ \\
\hline$\# 11$ & $(12)(34)(56)$ & $\# 24$ & $(34),(56),(134),(156)$ \\
\hline$\# 12$ & $(56),(34)$ & $\# 25$ & $(12)(34)(56),(234),(456),(125)$ \\
\hline$\# 13$ & $(12)(34)(56),(56)$ & & \\
\hline
\end{tabular}

Definition 12.3. Let $\sigma \in \Sigma(3,6)$ be a bipyramid cone. We write $\sigma=\left\langle e_{1}, e_{2}, e_{3}, e_{4}, e_{4}^{\prime}\right\rangle$, where $e_{1}, e_{2}, e_{3}$ are the vertices of the common base of the two pyramids, and split it into the following 12-cones

$$
\sigma_{i j k}=\left\langle e_{i}, e_{i}+e_{j}, e_{i}+e_{j}+e_{k}, e_{4}\right\rangle, \sigma_{i j k}^{\prime}=\left\langle e_{i}, e_{i}+e_{j}, e_{i}+e_{j}+e_{k}, e_{4}^{\prime}\right\rangle,
$$

where $\{i, j, k\}=\{1,2,3\}$. By also splitting the cones \#21, \#23, and \#24 as illustrated in Table 10, we obtain a refinement $\widehat{\Sigma}(3,6)$ of the fan $\Sigma(3,6)$. We define $\widehat{\mathbf{X}}(3,6)$ to be the closure of $\mathbf{X}(3,6)$ inside the smooth toric variety $Y_{\widehat{\Sigma}(3,6)}$. We have a commutative diagram

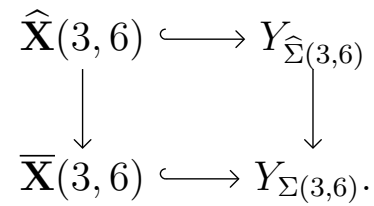

Since $\widehat{\Sigma}(3,6)$ refines $\Sigma(3,6)$, it follows by [Tev07, Proposition 2.5 ] that $\widehat{\mathbf{X}}(3,6)$ is a tropical compactification of $\mathbf{X}(3,6)$ and its morphism to $\overline{\mathbf{X}}(3,6)$ is toroidal.

Remark 12.4. Kapranov's compactification $\overline{\mathbf{X}}(3,6)$ is singular at exactly 15 isolated points each locally isomorphic to the cone over $\mathbb{P}^{1} \times \mathbb{P}^{2}$ ([Lux08, Theorem 4.2.4]). The 15 singular points are the strata that correspond to the bipyramid cones in $\Sigma(3,6)$. These singularities admit two small resolutions related by a flop: let $\overline{\mathbf{X}}_{1}(3,6)$ (resp. $\overline{\mathbf{X}}_{2}(3,6)$ ) be the small resolution with an exceptional locus isomorphic to $\mathbb{P}^{1}$ (resp. $\mathbb{P}^{2}$ ). There is also the blow up $\overline{\mathbf{X}}^{\prime}(3,6)$ of the cone singularities with exceptional divisor $\mathbb{P}^{1} \times \mathbb{P}^{2}$. All these compactifications are tropical and correspond to refinements of $\Sigma(3,6): \Sigma_{1}(3,6)$ is obtained by splitting each bipyramid into its two pyramids, $\Sigma_{2}(3,6)$ is obtained by adding to each bipyramid the segment that joins the two opposite vertices, and finally, $\Sigma^{\prime}(3,6)$ is the common refinement 
TABLE 10. The refinement $\widehat{\Sigma}(3,6)$ of the tropical Grassmannian.

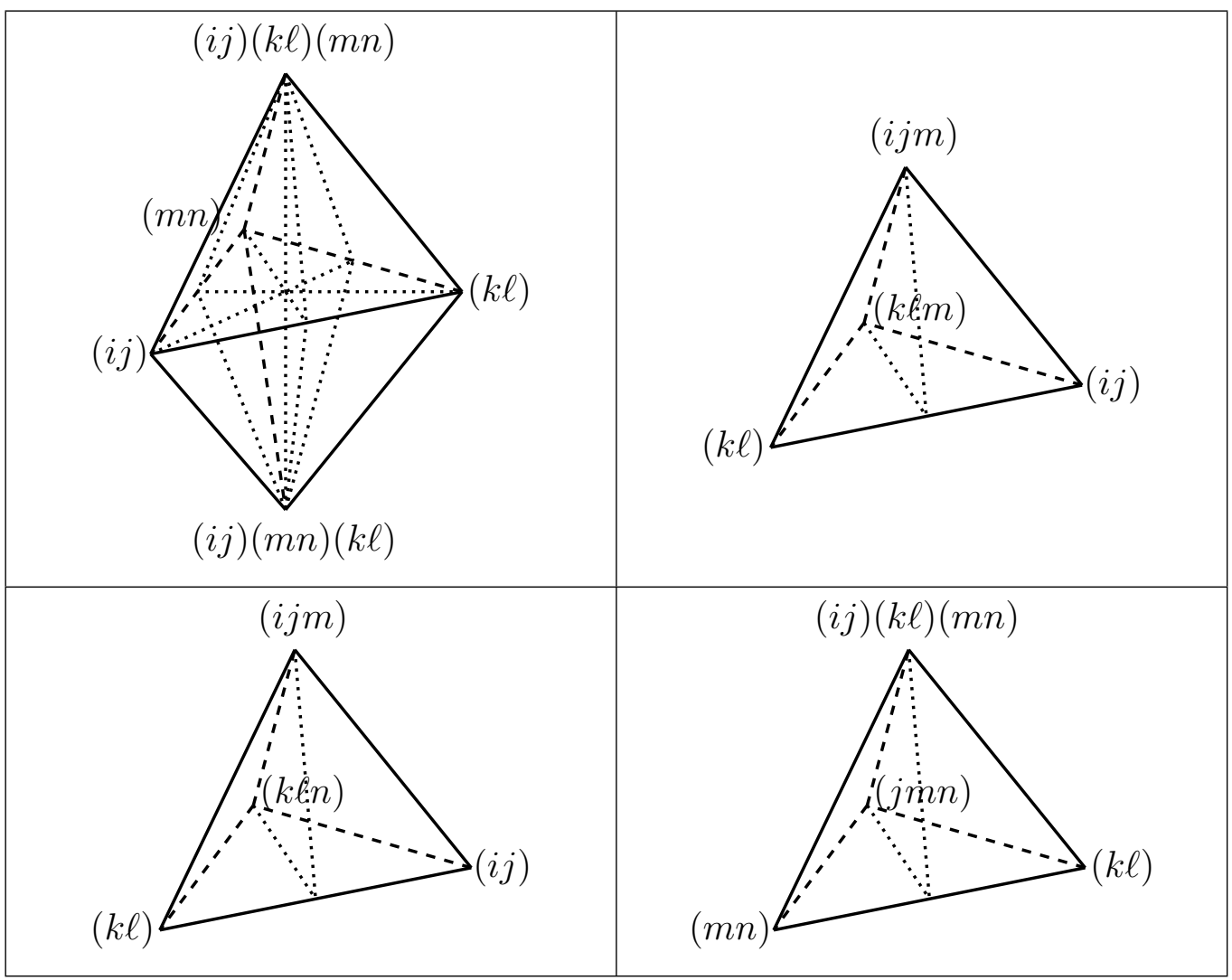

of $\Sigma_{1}(3,6)$ and $\Sigma_{2}(3,6)$. The space $\widehat{\mathbf{X}}(3,6)$ is obtained from $\overline{\mathbf{X}}^{\prime}(3,6)$ by blowing up the codimension 2 strata of type \#12. Summarizing, we have the following commutative diagram:

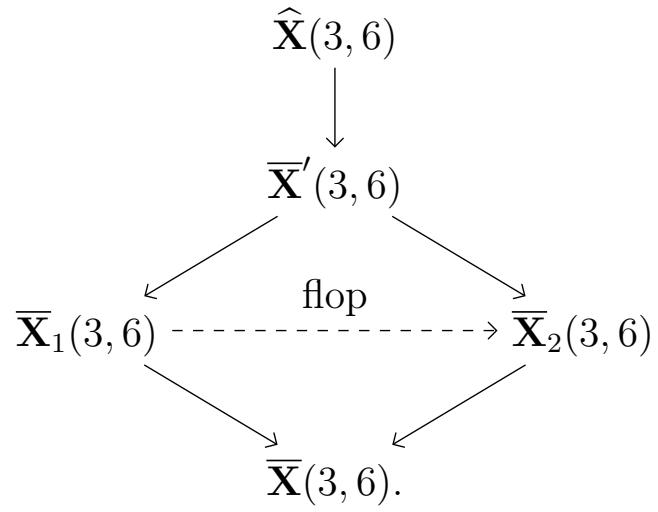

Proof of Theorem 1.7. Consider the birational map $f: \widehat{\mathbf{X}}(3,6) \rightarrow-\overline{\mathbf{X}}_{\mathrm{GP}}(3,6)^{\nu}$, which is an isomorphism over $\mathbf{X}(3,6)$. We first show that $f$ is an isomorphism over the open planar locus $\mathbf{U}_{\mathrm{GP}}(3,6) \subseteq \overline{\mathbf{X}}_{\mathrm{GP}}(3,6)^{\nu}$. Since $\mathbf{U}_{\mathrm{GP}}(3,6)$ is the union of the open subsets $\mathbf{U}_{\mathrm{GP}}^{i j k}$ for all triples 
of distinct indices, it suffices to show that $f$ is an isomorphism over $\mathbf{U}_{\mathrm{GP}}^{123}$. Recall from $\$ 11$ that $\mathbf{U}^{123} \subseteq \overline{\mathbf{X}}(3,6)$ is isomorphic to the open subset $\mathbf{V} \subseteq Y_{\mathcal{Q}_{3}^{\vee}}$. Let $\widehat{\mathbf{U}}^{123} \subseteq \widehat{\mathbf{X}}(3,6)$ be the preimage of $\mathbf{U}^{123}$ under the morphism $\widehat{\mathbf{X}}(3,6) \rightarrow \overline{\mathbf{X}}(3,6)$. Finally, let $\widehat{\mathbf{V}} \subseteq Y_{\widetilde{\mathcal{Q}}_{3}}$ be the open subset $A_{123}\left(\mathbf{U}_{\mathrm{GP}}^{123}\right)$ as in Definition 11.1 . We claim that $f$ induces an isomorphism $\widehat{\mathbf{U}}_{\mathrm{GP}}^{123} \cong \widehat{\mathbf{V}}$, so that we have a commutative diagram

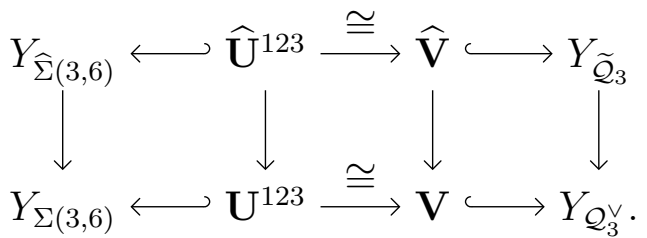

Since both spaces are obtained by toroidal blow ups of strata of the same variety $\mathbf{U}^{123}$, it suffices to prove that the strata match. We compare the refinements $\widehat{\Sigma}(3,6) \preccurlyeq \Sigma(3,6)$ and $\widetilde{\mathcal{Q}}_{3} \preccurlyeq \mathcal{Q}_{3}^{\vee}$. The maximal cones in $\Sigma(3,6)$ which are split in $\widehat{\Sigma}(3,6)$ and that correspond to points in $\mathbf{U}^{123}$ are the cones \#20, \#24, \#21 in Table 9. Under the isomorphism $\mathbf{U}^{123} \rightarrow \mathbf{V}$, these cones correspond to the cones of $\mathcal{Q}_{3}^{\vee}$ in the left column of Table 11. The cones in $\Sigma(3,6)$ are split as in Definition 12.3 , which matches the refinement given by the maximal cones in $\widetilde{\mathcal{Q}}_{3}$ in the right column of Table 11 . The three thickened segment lengths $x_{1}, x_{2}, x_{3}$ satisfy $x_{1}>x_{2}>x_{3}$. Different choices of inequality yield a total of 12 different cones in $\widetilde{\mathcal{Q}}_{3}$, which match the splitting in $\widehat{\Sigma}(3,6)$ of the bipyramid cone. This implies that $\widehat{\mathbf{U}}^{123} \cong \widehat{\mathbf{V}}$.

TABLE 11. On the left, subdivisions of $\mathbb{R}^{2}$ giving cones in $\mathcal{Q}_{3}^{\vee}$ which correspond to points in $\mathbf{V}$. These cones are refined by $\widetilde{\mathcal{Q}}_{3}$ on the right.

(1)


Next we study $f$ over the non-planar locus of $\overline{\mathbf{X}}_{\mathrm{GP}}(3,6)^{\nu}$. Let $x \in \overline{\mathbf{X}}_{\mathrm{GP}}(3,6) \backslash \mathbf{U}_{\mathrm{GP}}(3,6)$ and let $y \in \overline{\mathbf{X}}(3,6)$ be the image of $x$. Then $y$ is in the interior of the strata $\# 7$, \#2, \#10, or \#23 - these are the only degenerations in Figures 23, 24, and 25 which do not have a triple of lines in general linear position in each primary component. Each stratum \#7 is an isolated point of the non-planar locus. By the description of the fan $\widehat{\Sigma}(3,6)$, the morphism $\widehat{\mathbf{X}}(3,6) \rightarrow \overline{\mathbf{X}}(3,6)$ is a local isomorphism over this stratum. By normality, it suffices to show that $\overline{\mathbf{X}}_{\mathrm{GP}}(3,6)$ contains a unique point over each stratum \#7, which is proved in Lemma 12.5 .

Next we study $f$ over the preimage of the closed strata $\# 2=(i j k),(l m n)$ in $\overline{\mathbf{X}}(3,6)$, which contains the strata \#10 and \#23 in its interior. By [Lux08, Theorem 4.2.9], the boundary strata $(i j k)$ are isomorphic to $\overline{\mathrm{M}}_{0,6}$ and each stratum $\# 2=(i j k),(l m n)$ corresponds to a boundary stratum of $\overline{\mathrm{M}}_{0,6}$ isomorphic to $\mathbb{P}^{1} \times \mathbb{P}^{1}$, which we denote by $S$. The boundary divisors of type $(i j),(i k),(j k)$ restrict to $S$ giving three distinct parallel rulings, which are strata \#10. The pairwise intersections of these rulings with the analogous restrictions of the boundary divisors of type $(\ell m),(\ell n),(m n)$ give nine 0-dimensional boundary strata \#23. It follows from this analysis and the structure of the refinement $\widehat{\Sigma}(3,6)$ in Table 10 that the preimage $\widehat{S}$ of $S$ under the blow up $\widehat{\mathbf{X}}(3,6) \rightarrow \overline{\mathbf{X}}(3,6)$ is $\mathrm{Bl}_{9} S$, the blow up of $S$ at the nine 0-dimensional boundary strata \#23. Let $S_{\mathrm{GP}} \subseteq \overline{\mathbf{X}}_{\mathrm{GP}}(3,6)$ be the preimage of $S$. By Lemma 12.7, the morphism $S_{\mathrm{GP}} \rightarrow S$ induces a bijective normalization morphism $\widehat{S} \cong \mathrm{Bl}_{9} S \rightarrow S_{\mathrm{GP}}$. Take $x \in S_{\mathrm{GP}}$ and take any $\mathbf{a}: \operatorname{Spec}(K) \rightarrow \mathbf{B}_{6}$ with limit $x$. Denote by $z \in \widehat{S} \subseteq \widehat{\mathbf{X}}(3,6)$ the corresponding limit of $f^{-1} \circ \mathbf{a}$. In the proof of Lemma 12.7 we showed that $x$ only depends on $z$ and not on the choice of $\mathbf{a}$. This shows that $f$ is regular at $z$ by [GG14, Theorem 7.3]. Since $f$ is finite and birational, $\overline{\mathbf{X}}_{\mathrm{GP}}(3,6)^{\nu} \cong \widehat{\mathbf{X}}(3,6)$.

Lemma 12.5. $\overline{\mathbf{X}}_{\mathrm{GP}}(3,6)$ contains a unique point over each stratum $\# 7$ in $\overline{\mathbf{X}}(3,6)$.

Proof. Consider the following one-parameter family a $(t)$ of six points in $\mathbb{P}^{2}$ :

$$
\begin{gathered}
a_{1}(t)=[1: 0: 0], a_{2}(t)=[0: 1: 0], a_{3}(t)=[0: 0: 1], a_{4}(t)=[1: 1: 1], \\
a_{5}(t)=\left[c_{1} t: 1: 1+c_{2} t\right], a_{6}(t)=\left[1+c_{3} t: 1: c_{4} t\right],
\end{gathered}
$$

where $c_{1}, \ldots, c_{4} \in R \backslash\{0\}$. Let $\lambda_{1}, \ldots, \lambda_{4}$ be the valuations of $c_{1}, \ldots, c_{4}$, respectively. The limit points for $t=0$ are the double points of the line arrangement in Figure 20 given by:

$$
\ell_{126}=\left\{x_{3}=0\right\}, \ell_{145}=\left\{x_{2}=x_{3}\right\}, \ell_{235}=\left\{x_{1}=0\right\}, \ell_{346}=\left\{x_{1}=x_{2}\right\} .
$$

The stable lattices and the corresponding stabilized quadruples are the following:

- $L_{0}=e_{1} R+e_{2} R+e_{3} R$ stabilizes $(1,2,3,4),(1,3,5,6),(2,4,5,6)$;

- $L_{1}=t^{\lambda_{1}+1} e_{1} R+e_{2} R+e_{3} R$ stabilizes $(1,2,3,5),(2,3,4,5),(2,3,5,6)$;

- $L_{2}=e_{1} R+e_{2} R+t^{\lambda_{4}+1} e_{3} R$ stabilizes $(1,2,3,6),(1,2,4,6),(1,2,5,6)$;

- $L_{3}=e_{1} R+t^{\lambda_{2}+1} e_{2} R+\left(e_{2}+e_{3}\right) R$ stabilizes $(1,2,4,5),(1,3,4,5),(1,4,5,6)$;

- $L_{4}=t^{\lambda_{3}+1} e_{1} R+\left(e_{1}+e_{2}\right) R+e_{3} R$ stabilizes $(1,3,4,6),(2,3,4,6),(3,4,5,6)$.

We now compute the 6 -pointed degenerate central fiber $\mathbb{P}\left(\Sigma_{\mathbf{a}}\right)_{\mathbb{k}}$. Let us start by observing that the induced linear spaces on $\mathbb{P}\left(L_{0}\right)_{\mathbb{k}}$ are given by

$$
W_{L_{0}}\left(L_{1}\right)=\left\{x_{1}=0\right\}, W_{L_{0}}\left(L_{2}\right)=\left\{x_{3}=0\right\}, W_{L_{0}}\left(L_{3}\right)=\left\{x_{2}=x_{3}\right\}, W_{L_{0}}\left(L_{4}\right)=\left\{x_{1}=x_{2}\right\} .
$$




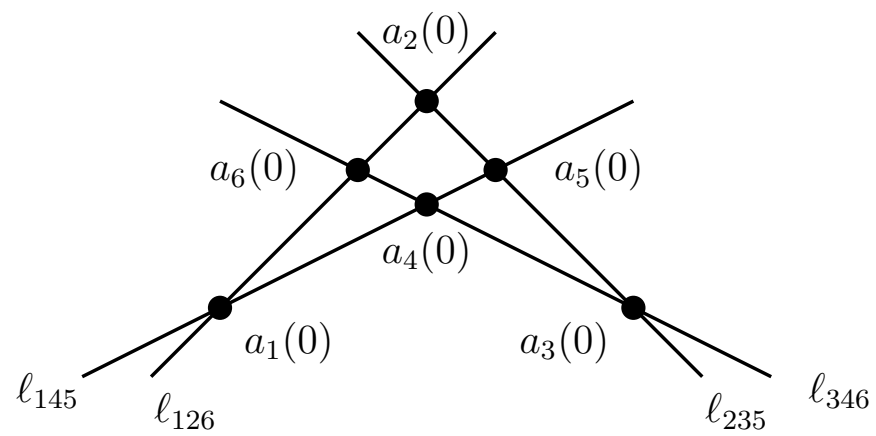

Figure 20. Degeneration of six points in $\mathbb{P}^{2}$.

In particular the primary component in $\mathbb{P}\left(\Sigma_{\mathbf{a}}\right)_{\mathbb{k}}$ corresponding to $L_{0}$ is isomorphic to $\mathbb{P}^{2}$. On the other hand, let $i, j$ be distinct nonzero indices. Then $W_{L_{i}}\left(L_{0}\right)$ is a point by Remark 5.5 (note that $L_{i} \subseteq L_{0}$ and $W_{L_{0}}\left(L_{i}\right)$ is a line). Moreover $W_{L_{i}}\left(L_{j}\right)$ is a line again by Remark 5.5 $\left(L_{i} \nsubseteq L_{j}\right.$ and $\left.L_{j} \nsubseteq L_{i}\right)$. So the primary component $S_{i}$ in $\mathbb{P}\left(\Sigma_{\mathbf{a}}\right)_{\mathbb{k}}$ corresponding to $L_{i}$ is isomorphic to $\mathbb{F}_{1}$. The secondary components of $\mathbb{P}\left(\Sigma_{\mathbf{a}}\right)_{\mathbb{k}}$ can be understood as follows. If $i, j$ are distinct nonzero indices, then the central fiber $\mathbb{P}\left(L_{0}, L_{i}, L_{j}\right)_{\mathbb{k}}$ is as shown in Figure 4 , and there is a unique secondary component $T_{i j}$ isomorphic to $\mathbb{P}^{1} \times \mathbb{P}^{1}$. Note that the center of the blow up $\mathbb{P}\left(\Sigma_{\mathbf{a}}\right) \rightarrow \mathbb{P}\left(L_{0}, L_{i}, L_{j}\right)$ does not intersect $T_{i j}$. In conclusion, $\mathbb{P}\left(\Sigma_{\mathbf{a}}\right)_{\mathbb{k}}$ has six secondary components $T_{i j}$ isomorphic to $\mathbb{P}^{1} \times \mathbb{P}^{1}$, each one glued along two of its incident rulings to a fiber in each $S_{i}, S_{j}$. The components $S_{1}, \ldots, S_{4}$ are then glued along their exceptional curves to $\mathbb{P}^{2}$ along the lines $W_{L_{0}}\left(L_{1}\right), \ldots, W_{L_{0}}\left(L_{4}\right)$ respectively. The resulting surface $\mathbb{P}\left(\Sigma_{\mathbf{a}}\right)_{\mathbb{k}}$ is pictured in Figure 21. It is independent from $c_{1}, \ldots, c_{4} \in R \backslash\{0\}$.

Let us determine the limits $\bar{a}_{1}(0), \ldots, \bar{a}_{6}(0) \in \mathbb{P}\left(\Sigma_{\mathbf{a}}\right)_{\mathbb{k}}$. Under the birational morphism $\mathbb{P}\left(\Sigma_{\mathbf{a}}\right) \rightarrow \mathbb{P}\left(L_{0}\right)$, the irreducible components of $\mathbb{P}\left(\Sigma_{\mathbf{a}}\right)_{\mathbb{k}}$ mapping to $\ell_{126}$ (resp. $\left.\ell_{145}\right)$ are $S_{2}, T_{12}, T_{23}, T_{24}$ (resp. $\left.S_{3}, T_{13}, T_{23}, T_{34}\right)$. This implies that the limit point $\bar{a}_{1}^{L_{0}}(0)=\ell_{126} \cap \ell_{145}$ is on $T_{23}$. By the same reasoning, we have that

$$
\bar{a}_{1}(0) \in T_{23}, \bar{a}_{2}(0) \in T_{12}, \bar{a}_{3}(0) \in T_{14}, \bar{a}_{4}(0) \in T_{34}, \bar{a}_{5}(0) \in T_{13}, \bar{a}_{6}(0) \in T_{24} .
$$

Finally, we count the moduli associated to the choice of the six markings on the $T_{i j}$ components of $\mathbb{P}\left(\Sigma_{\mathbf{a}}\right)_{\mathbb{k}}$. We show that we can find an appropriate automorphism of the surface $\mathbb{P}\left(\Sigma_{\mathbf{a}}\right)_{\mathbb{k}}$ that fixes the six markings. Consider $S_{1}$, which is glued to $T_{12}, T_{13}, T_{14}$ along three rulings $f_{12}, f_{13}, f_{14}$ respectively. The automorphism group of $S_{1}$ that preserves the $(-1)$-section and the rulings $f_{12}, f_{13}, f_{14}$ is three dimensional, and we can choose one of such automorphisms $\varphi_{1}$ that fixes the images on $S_{1}$ of the limits $\bar{a}_{2}(0) \in T_{12}, \bar{a}_{3}(0) \in$ $T_{14}, \bar{a}_{5}(0) \in T_{13}$. Consider the analogous automorphisms $\varphi_{2}, \varphi_{3}, \varphi_{4}$ for $S_{2}, S_{3}, S_{4}$ respectively. We can choose an appropriate $\psi_{i j} \in \operatorname{Aut}\left(T_{i j}\right)$ acting on the two rulings in such a way that $\psi_{i j}, \varphi_{k}, \operatorname{id}_{\mathbb{P}\left(L_{0}\right)_{\mathbb{k}}}$ glue to a global automorphism of $\mathbb{P}\left(\Sigma_{\mathbf{a}}\right)_{\mathbb{k}}$ which fixes the six markings.

Next we consider the non-planar locus in $\overline{\mathbf{X}}_{\mathrm{GP}}(3,6)^{\nu}$ dominating the closed stratum \#2 in $\overline{\mathbf{X}}(3,6)$. First we classify degenerations parametrized by it. 


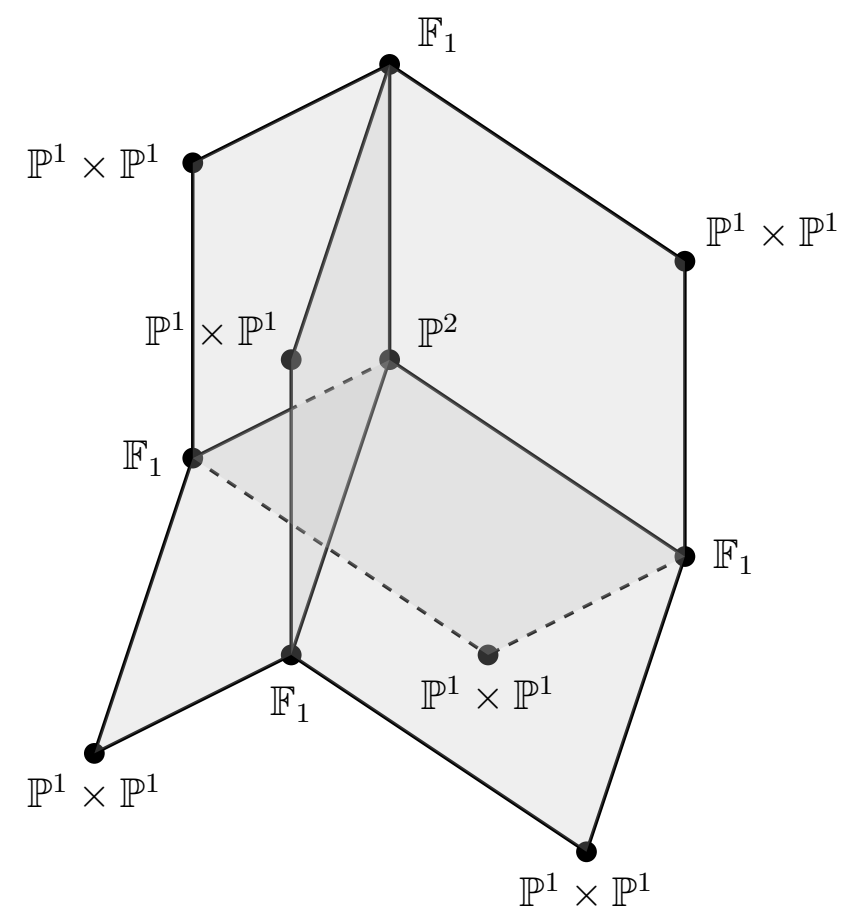

Figure 21. Dual complex of the degeneration discussed in Lemma 12.5 .

Lemma 12.6. The 6-pointed degenerations of $\mathbb{P}^{2}$ parametrized by points in $\overline{\mathbf{X}}_{\mathrm{GP}}(3,6)$ over the (closed) stratum of type \#2 in $\overline{\mathbf{X}}(3,6)$ are depicted in figures \#2, 10, 23, 23.1 in Table 13.

Proof. Let $x \in \overline{\mathbf{X}}_{\mathrm{GP}}(3,6)$ be a point over a point $y \in \overline{\mathbf{X}}(3,6)$ in the (closed) stratum $\# 2$. From the possible Kapranov's degenerations \#2, \#10, \#23 parametrized by $y$, which can be found in [Ale15, Figures 5.12 and 5.13], we conclude that all stable lattices are contained in one apartment. For clarity, let us point out that this apartment is not determined by a triple of sections as for degenerations parametrized by the planar locus. Instead, the point $y$ is in the image of the planar locus in $\overline{\mathbf{X}}(3,7)$ with respect to the forgetful map. By considering combinatorial types of the dual spiders giving the degenerations of $\left(\mathbb{P}^{2}\right)^{\vee}$ parametrized by $y$ and the corresponding usual spiders, we compute the resulting degenerations of $\mathbb{P}^{2}$, taking into account that stretching the spiders may yield different degenerations. This is summarized in Table 12. For each pair, on the left we give dual spiders in one apartment for the degenerations \#2,10,23 of six lines in $\left(\mathbb{P}^{2}\right)^{\vee}$, and on the right the corresponding spiders for the degenerations of six points in $\mathbb{P}^{2}$. Finally, we add to these degenerations of $\mathbb{P}^{2}$ six marked points as prescribed by Lemma 7.13 .

Lemma 12.7. Let $S \subseteq \overline{\mathbf{X}}(3,6)$ be a (closed) stratum of type $\# 2$ and let $S_{\mathrm{GP}} \subseteq \overline{\mathbf{X}}_{\mathrm{GP}}(3,6)$ be its preimage. The birational morphism $S_{\mathrm{GP}} \rightarrow S$ induces a bijective normalization morphism $\mathrm{Bl}_{9} S \rightarrow S_{\mathrm{GP}}$, where $\mathrm{Bl}_{9} S$ is the blow up of $S$ at the nine double points (strata \#23) of the arrangement of six rulings (strata \#10). 
TABLE 12. Dual spiders for the degenerations \#2,10, 23 of six lines in $\left(\mathbb{P}^{2}\right)^{\vee}$, and the corresponding spiders for the degenerations of six points $\mathbb{P}^{2}$.

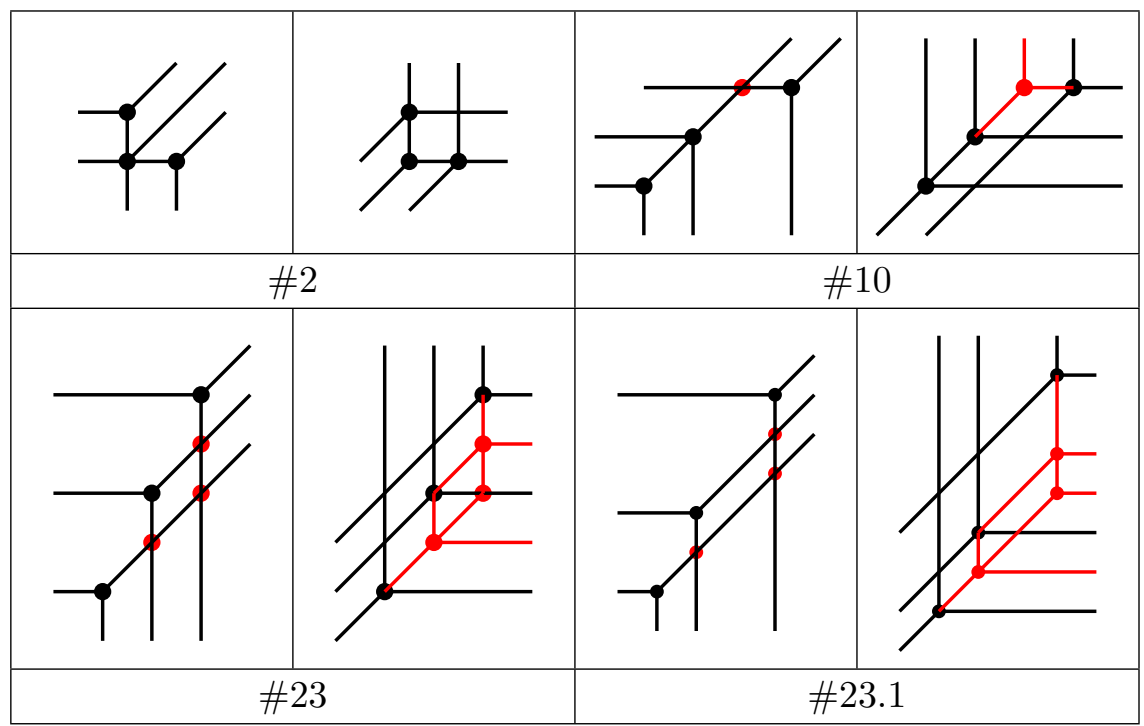

Proof. Consider a general one-parameter family of six lines

$$
\mathbf{a}(t)=\left([1: 0: 0],[0: 1: 0],[0: 0: 1],[1: 1: 1],\left[1: c_{1}: c_{2} t\right],\left[1: 1+c_{3} t: c_{4}\right]\right)
$$

in $\left(\mathbb{P}^{2}\right)^{\vee}$ limiting to a point $y \in \overline{\mathbf{X}}(3,6)$ in the open stratum \#2. Here $c_{1}, \ldots, c_{4} \in R \backslash\{0\}$ and $c_{1}(0), c_{4}(0) \neq 0,1$. It can also be viewed as a general one-parameter family of six points in $\mathbb{P}^{2}$ with limit $x \in \overline{\mathbf{X}}_{\mathrm{GP}}(3,6)^{\nu}$. We want to show that $x$ only depends on $y$, and not on the choice of the arc a. The degeneration of $\mathbb{P}^{2}$ parametrized by $x$ is pictured in Table 13 , \#2, and it only depends on the two cross-ratios we are about to describe. The limits in $\mathbb{P}^{2}$ of the six points in a lie on the two lines $x_{3}=0$ and $x_{1}-x_{2}=0$, which intersect at $[1: 1: 0]$. So consider the quadruple $[1: 0: 0],[0: 1: 0],\left[1: c_{1}(0): 0\right],[1: 1: 0]$ on the first line, and $[1: 1: 1],[0: 0: 1],\left[1: 1: c_{4}(0)\right],[1: 1: 0]$ on the second line. Their cross-ratios are $c_{1}(0)$ and $c_{4}(0)$ respectively. On the other hand, let $\ell_{1}, \ldots, \ell_{6}$ be the lines in $\left(\mathbb{P}^{2}\right)^{\vee}$ corresponding to the six points in $\mathbf{a}$. In the limit, $\ell_{1}, \ell_{2}, \ell_{5}$ (resp. $\left.\ell_{3}, \ell_{4}, \ell_{6}\right)$ pass through $[0: 0: 1]$ (resp. $[1:-1: 0])$. Let $\ell$ be the line passing through $[0: 0: 1]$ and $[1:-1: 0]$. The stable pair parametrized by $y \in \overline{\mathbf{X}}(3,6)$ is pictured in Figure 24, \#2 (note that the labels used for the lines in this proof do not match the ones in the figure), and the limits of $\ell_{1}, \ell_{2}, \ell_{5}, \ell$ and $\ell_{3}, \ell_{4}, \ell_{6}, \ell$ determine four points on each one of the two irreducible $\mathbb{P}^{1}$ in the double locus. The degeneration parametrized by $y \in \overline{\mathbf{X}}(3,6)$ only depends on the cross-ratios of these two quadruples, which are equal to $c_{1}(0)$ and $c_{4}(0)$ respectively. In conclusion, $x$ only depends on $y$, and not on the tangent direction of the arc limiting to $y$.

We apply the same strategy for a point $x \in \overline{\mathbf{X}}_{\mathrm{GP}}(3,6)^{\nu}$ in the preimage of the interior of the stratum \#10. The general one-parameter family of six points in $\mathbb{P}^{2}$ with limit $x$ is

$$
\mathbf{a}(t)=\left([1: 0: 0],[0: 1: 0],[0: 0: 1],[1: 1: 1],\left[1: c_{1}: c_{2} t\right],\left[1: 1+c_{3} t: c_{4} t\right]\right),
$$


where $c_{1}, \ldots, c_{4} \in R \backslash\{0\}$ and $c_{1}(0) \neq 0,1$. Viewing this as a family of lines we obtain a limit $y \in \overline{\mathbf{X}}(3,6)$. The degeneration parametrized by $x$ depends on one cross-ratio, which is determined by $[1: 0: 0],[0: 1: 0],\left[1: c_{1}(0): 0\right],[1: 1: 0]$ on the line $x_{3}=0$ and equals $c_{1}(0)$. Consider the corresponding degeneration of six lines parametrized by $y \in \overline{\mathbf{X}}(3,6)$, which is pictured in Figure 24, \#10. This degeneration is determined by the cross-ratio of four points on the gluing locus between $\mathbb{P}^{1} \times \mathbb{P}^{1}$ and $\mathbb{F}_{1}$. These four points are cut out by another curve in the double locus and the limits of $\ell_{1}, \ell_{2}, \ell_{5}$. Their cross-ratio is equal to $c_{1}(0)$.

The general one-parameter family limiting to $y \in \overline{\mathbf{X}}(3,6)$ in the (closed) stratum \#23 is given by

$$
\mathbf{a}(t)=\left([1: 0: 0],[0: 1: 0],[0: 0: 1],[1: 1: 1],\left[1: 1+c_{1} t: c_{2} t\right],\left[1: 1+c_{3} t: c_{4} t\right]\right),
$$

where $c_{1}, \ldots, c_{4} \in R \backslash\{0\}$. Here we interpret the arc as a one-parameter family of lines $\ell_{1}, \ldots, \ell_{6}$ in $\left(\mathbb{P}^{2}\right)^{\vee}$. Consider the cross-ratio morphisms $\gamma_{1}, \gamma_{2}: \overline{\mathbf{X}}(3,6) \rightarrow \mathbb{P}^{1}$ given by restricting the lines $\ell_{1}, \ell_{2}, \ell_{5}, \ell_{6}$ to $\ell_{3}$ and $\ell_{3}, \ell_{4}, \ell_{5}, \ell_{6}$ to $\ell_{1}$. Then $\left(\gamma_{1}, \gamma_{2}\right)$ induces a local isomorphism near $y$ between $S$ and $\mathbb{P}^{1} \times \mathbb{P}^{1}$. A simple calculation shows that the limits as $t \rightarrow 0$ of the derivatives with respect to $t$ of $\gamma_{1}(\mathbf{a}(t))$ and $\gamma_{2}(\mathbf{a}(t))$ are equal to $c_{3}(0)-c_{1}(0)$ and $c_{4}(0)-c_{2}(0)$ respectively. We claim that the limit as $t \rightarrow 0$ of the arc $\mathbf{a}(t)$ in $\overline{\mathbf{X}}_{\mathrm{GP}}(3,6)$ is uniquely determined by the point $\left[c_{1}(0)-c_{3}(0): c_{4}(0)-c_{2}(0)\right] \in \mathbb{P}^{1}$. In $\mathbb{P}^{2}$, consider the cross-ratio of the following four points on the line $x_{2}=0:[1: 0: 0],[0: 0: 1],[1: 0: 1]$, and the intersection of $x_{2}=0$ with the line spanned by $a_{5}(t), a_{6}(t)$, which is given by $\left[c_{1}-c_{3}:\left(c_{4}-c_{2}\right)+\left(c_{1} c_{4}-c_{2} c_{3}\right) t\right]$. Denote by $\beta$ its limit for $t \rightarrow 0$.

This cross-ratio manifests itself in the 6 -pointed degeneration $X$ of $\mathbb{P}^{2}$ parametrized by the limit $x \in \overline{\mathbf{X}}_{\mathrm{GP}}(3,6)^{\nu}$ over the (closed) stratum $\# 23$ in $\mathbf{X}(3,6)$ as follows. Assume that the degenerate surface $X$ parametrized by $x$ is obtained by gluing five copies of $\mathbb{F}_{1}$, three copies of $\mathbb{P}^{1} \times \mathbb{P}^{1}$, and one copy of the blow up of $\mathbb{P}^{2}$ at two points, and that the limit points are as shown in Figure 22. Denote these surfaces by $F_{1}, \ldots, F_{5}, P_{1}, P_{2}, P_{3}, B$ respectively. On $F_{1}$ (resp. $F_{5}$ ) let $f_{1}, f_{2}$ (resp. $f_{3}, f_{4}$ ) be the rulings passing through the points marked by 1,2 (resp. 3,4). These induce other two rulings $f_{1}^{\prime}, f_{2}^{\prime}$ (resp. $f_{3}^{\prime}, f_{4}^{\prime}$ ) on $F_{2}$ (resp. $F_{4}$ ). The rulings $f_{1}^{\prime}, \ldots, f_{4}^{\prime}$ intersect $F_{3}$ in four points $p_{1}, \ldots, p_{4}$, respectively. Let $r_{5}$ (resp. $r_{6}$ ) be the ruling in $P_{1}$ (resp. $P_{3}$ ) passing through the point marked by 5 (resp. 6) and intersecting $B$ into a point $q_{5}$ (resp. $q_{6}$ ). There is a unique line $\ell$ in $B \cong \mathrm{Bl}_{2} \mathbb{P}^{2}$ disjoint from the two exceptional divisors which passes through $q_{5}$ and $q_{6}$. This line $\ell$ intersects in a point $p$ the strict transform in $B$ of the line passing through the two points blown up. The point $p$ lies in the exceptional divisor of $F_{3}$, and it is contained in a unique ruling $f$. Now consider the line $\ell_{13}$ (resp. $\ell_{24}$ ) in $F_{3}$ spanned by $p_{1}$ and $p_{3}$ (resp. $p_{2}$ and $p_{4}$ ). On the line $\ell_{13}$ we have four distinct points $p_{1}, p_{3}, \ell_{13} \cap \ell_{24}, \ell_{13} \cap f$, and denote their cross-ratio by $\beta^{\prime}$ (this construction is reproduced in Figure 22). Note that $F_{3}$ is the primary component corresponding to the standard lattice $L_{0}=e_{1} R+e_{2} R+e_{3} R$ with respect to which the arc $\mathbf{a}(t)$ was given above, and $\beta$ was constructed inside $\mathbb{P}\left(L_{0}\right)_{\mathbb{k}} \cong \mathbb{P}^{2}$ in the same way we did for $\beta^{\prime}$. Therefore, $\beta^{\prime}=\beta \neq 0, \infty$, and it characterizes the isomorphism type of the 6 -pointed degeneration $X$. The extreme cases $\beta=0, \infty$ correspond to the following further degenerations of $X$ in Table 13 , \#23.1. The surface $F_{3} \subseteq X$ further breaks into the gluing of a surface $P \cong \mathbb{P}^{1} \times \mathbb{P}^{1}$, and a surface $T \cong \mathbb{P}^{2}$ along a ruling of $P$ and a line in $T$. Without loss of generality, assume that $P$ 
is glued with $F_{2}$. In this case the line $\ell_{13}$ breaks into two irreducible components, and the analogues of the points $p_{1}, p_{3}, \ell_{13} \cap \ell_{24}, \ell_{13} \cap f$ split into two groups $\left\{p_{1}, \ell_{13} \cap f\right\},\left\{p_{3}, \ell_{13} \cap \ell_{24}\right\}$, where $p_{1}, \ell_{13} \cap f$ lie on the surface $P$ and $p_{3}, \ell_{13} \cap \ell_{24}$ on $T$. This together with the other degeneration where $P$ is glued to $F_{4}$ instead, correspond, up to order, to the two cross-ratios $\beta=0, \infty$.

This shows that we have morphisms $\mathrm{Bl}_{9} S \rightarrow S_{\mathrm{GP}} \rightarrow S$, where the first morphism is a bijective normalization.

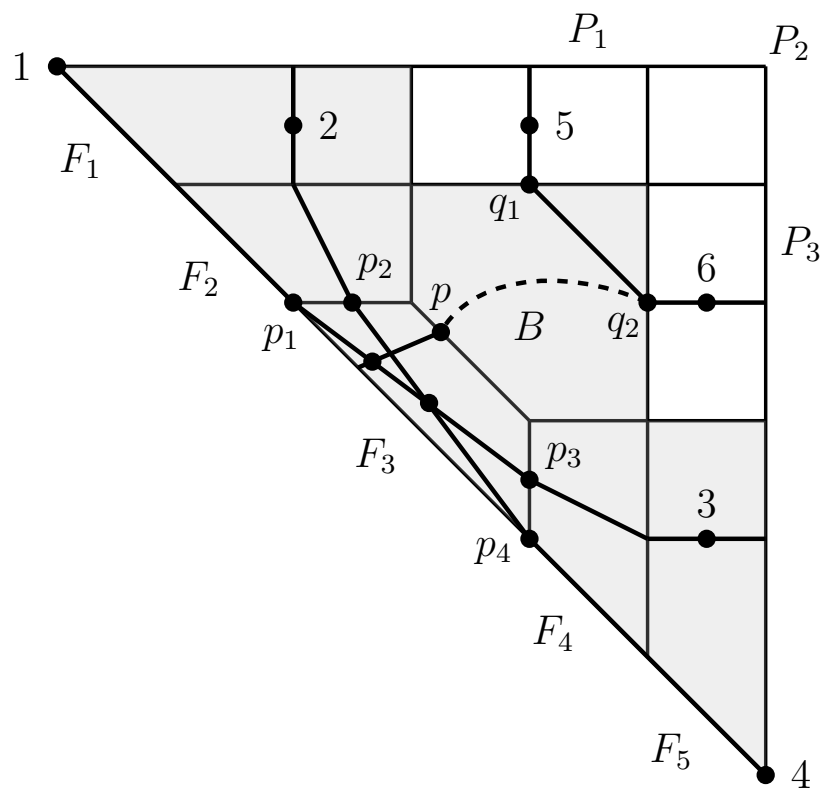

Figure 22. Cross-ratio $\beta$ in a degeneration of six points in $\mathbb{P}^{2}$ parametrized by a point in $\overline{\mathbf{X}}_{\mathrm{GP}}(3,6)^{\nu}$ over the (closed) stratum $\# 23$ in $\overline{\mathbf{X}}(3,6)$.

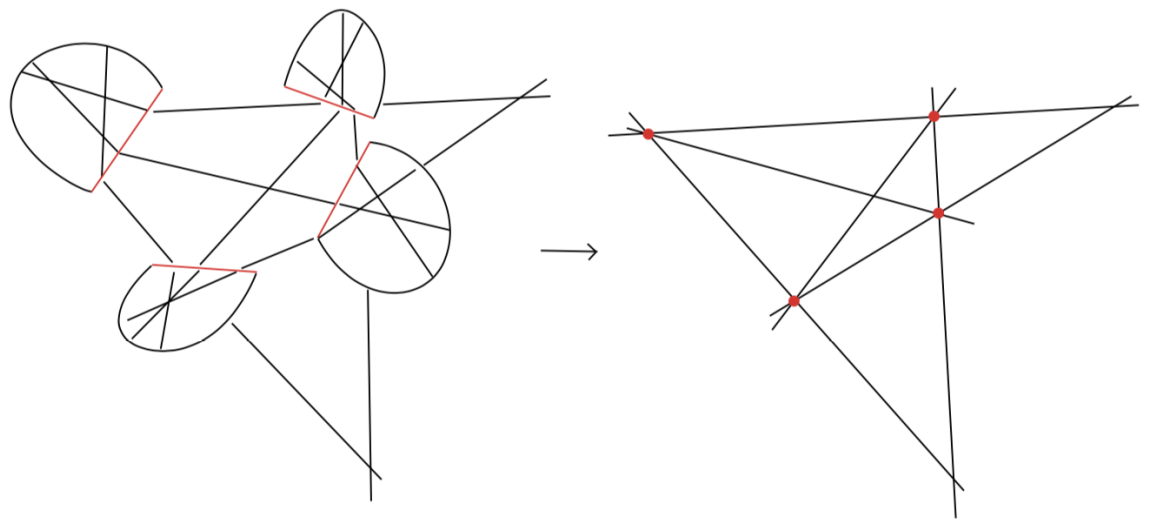

Figure 23. (From [Ale15, Figure 5.11] with permission of the author.) 
TABLE 13. Non-planar degenerations parametrized by $\overline{\mathbf{X}}_{\mathrm{GP}}(3,6)$.

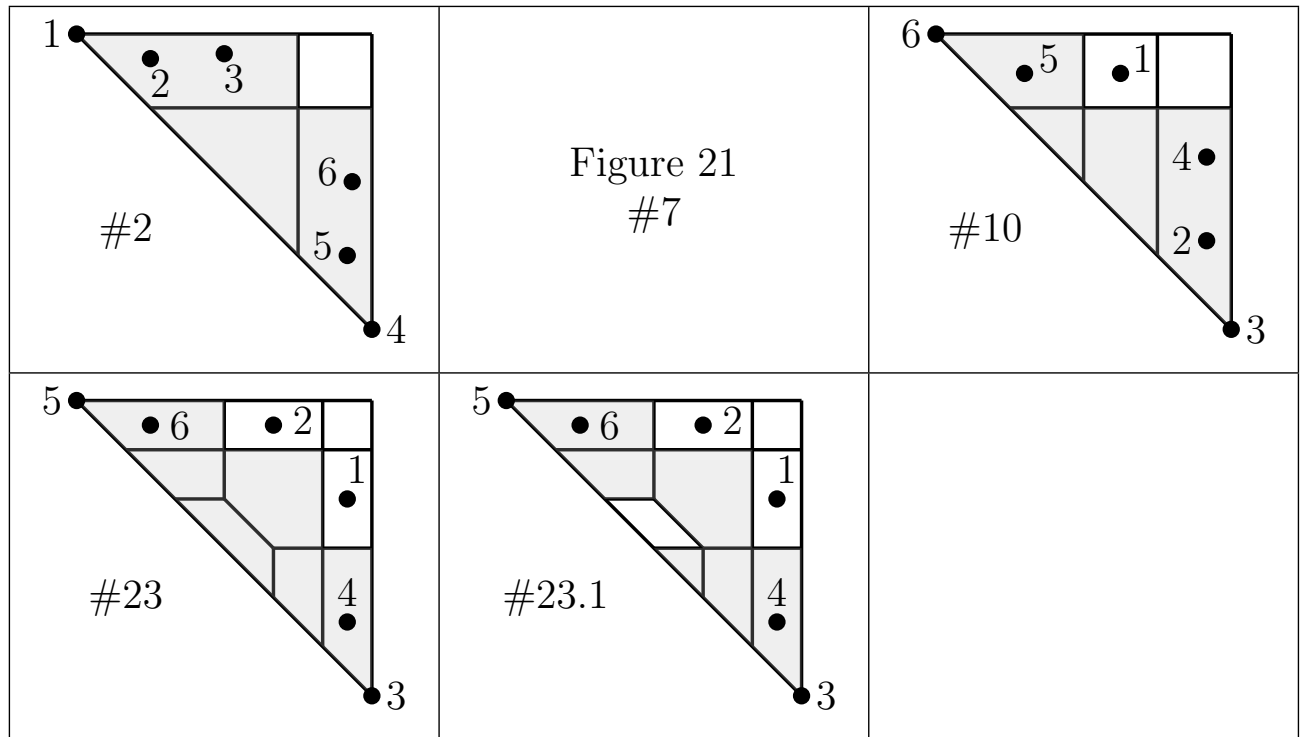

TABLE 14. Planar degenerations parametrized by $\overline{\mathbf{X}}_{\mathrm{GP}}(3,6)$.

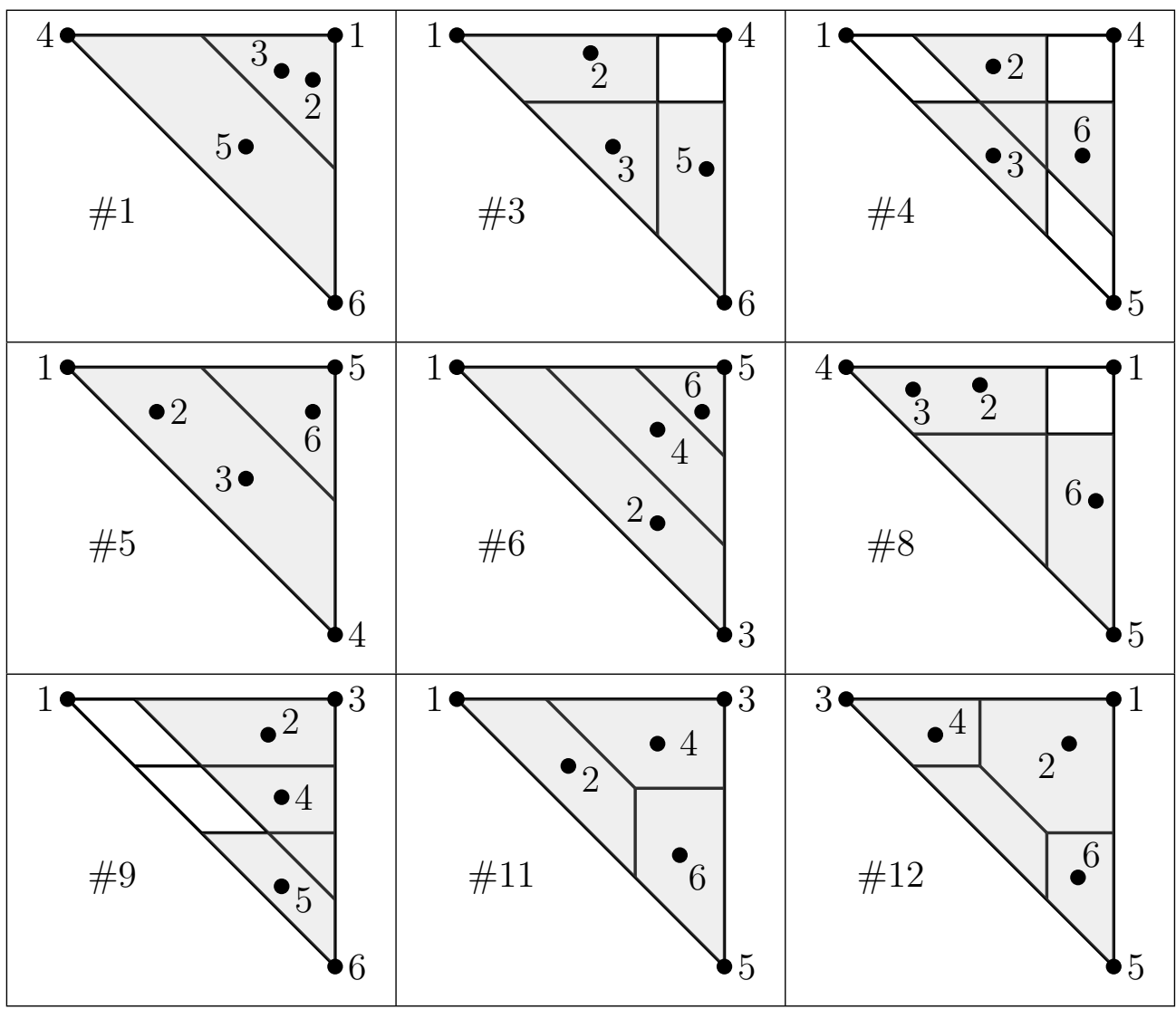


TABLE 15. Planar degenerations parametrized by $\overline{\mathbf{X}}_{\mathrm{GP}}(3,6)$, continued.

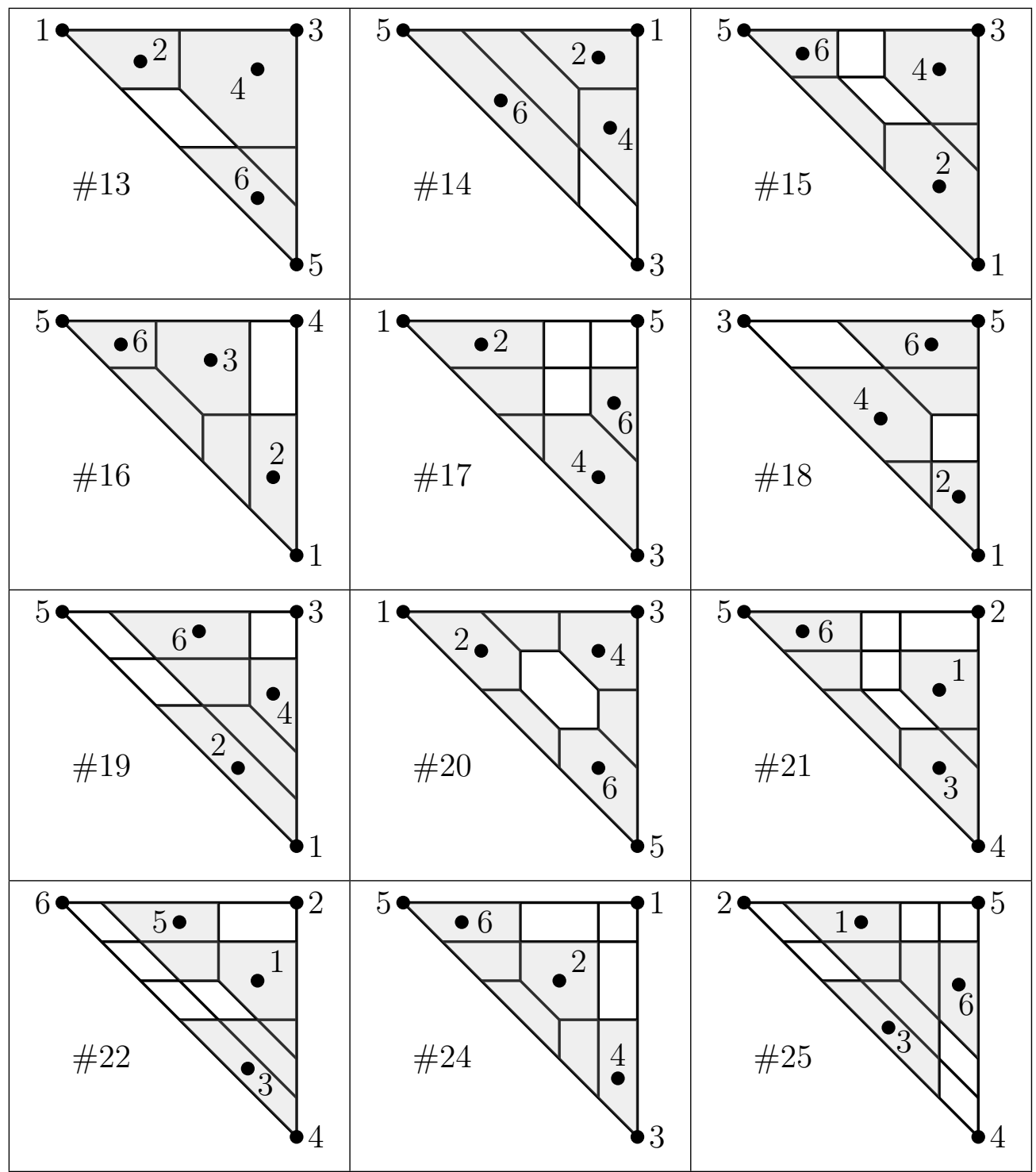


TABLE 16. Further planar degenerations that come from the stretches in Table 6 .

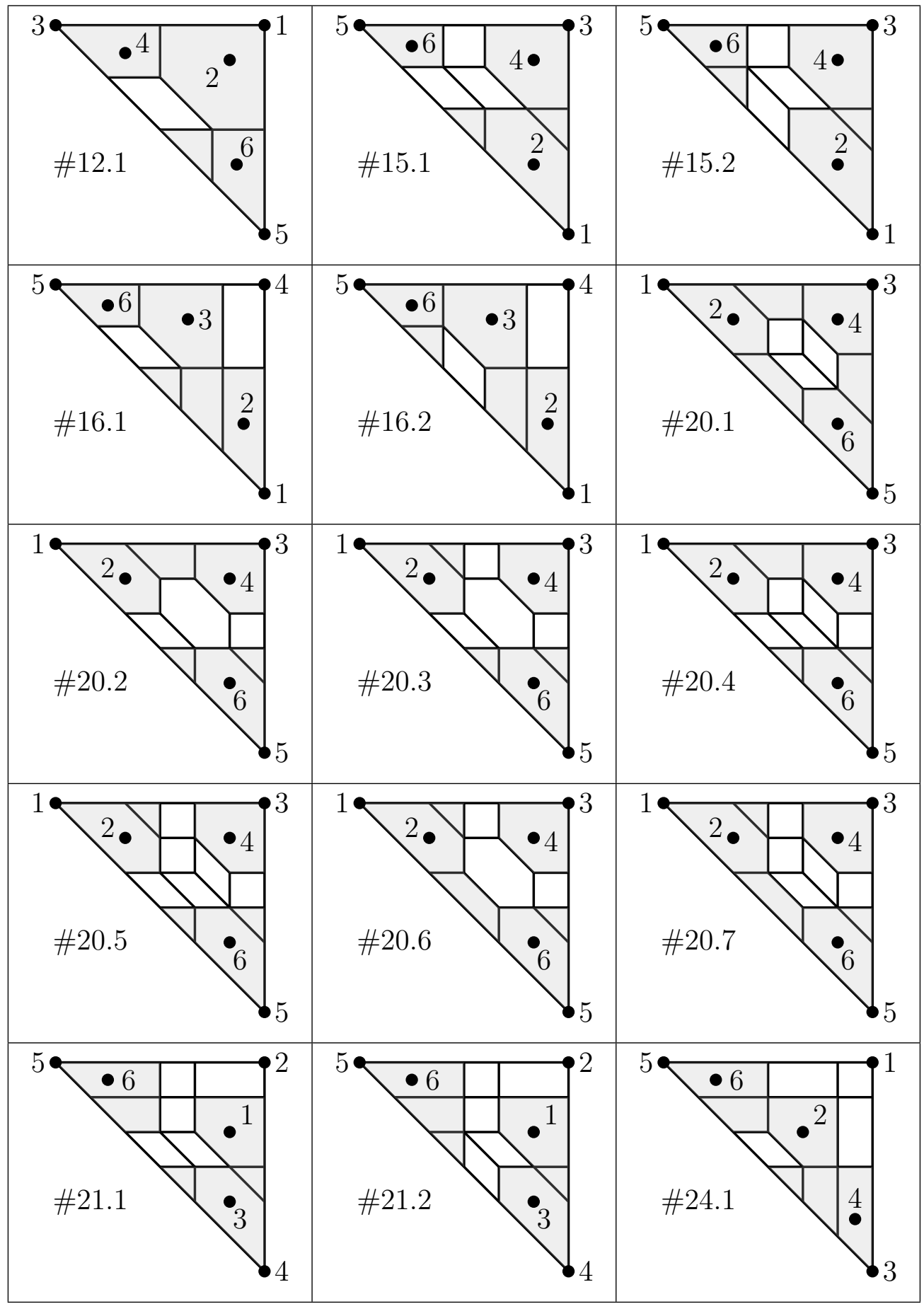



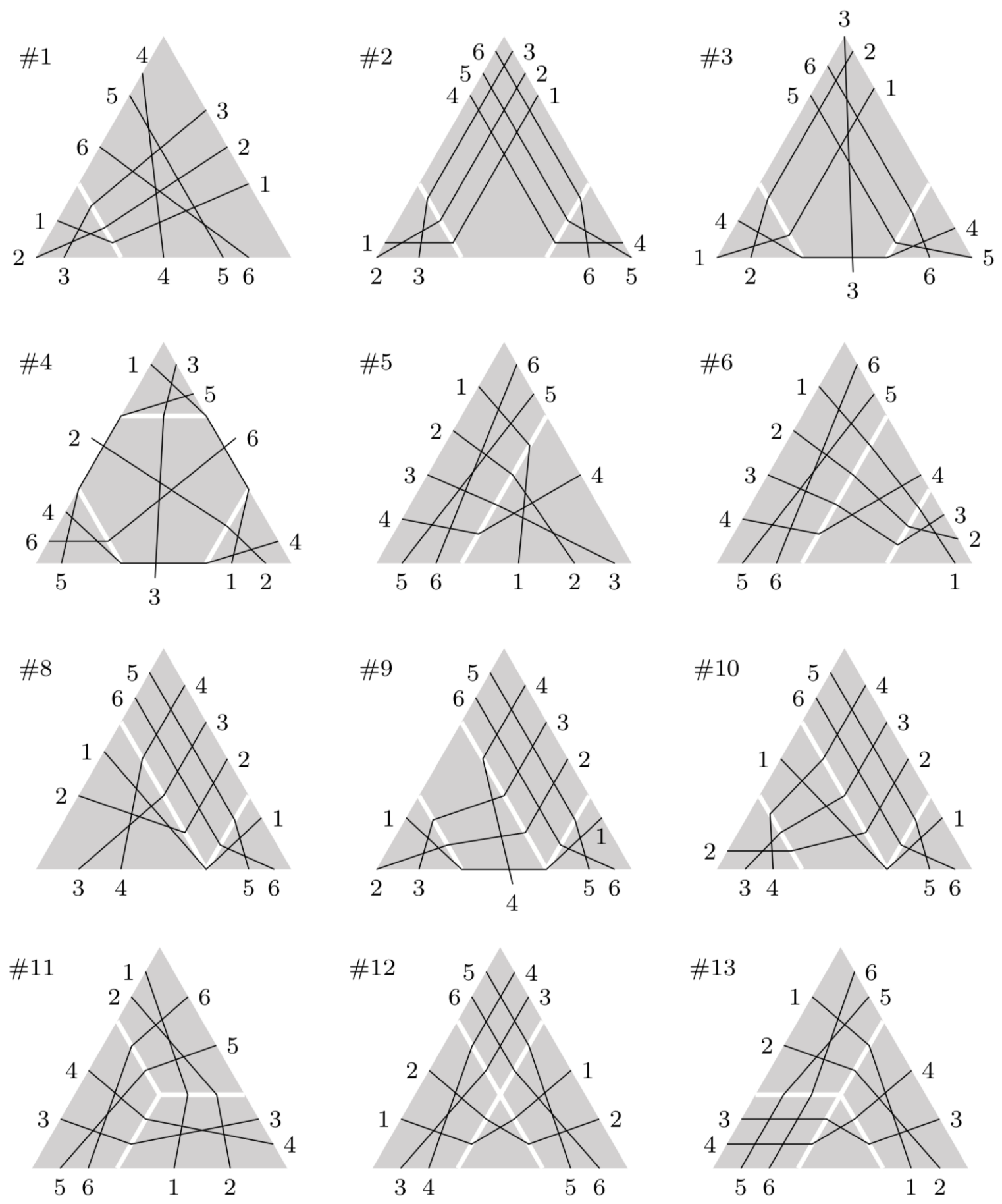

FiguRE 24. (From [Ale15, Figure 5.12] with permission of the author.) 

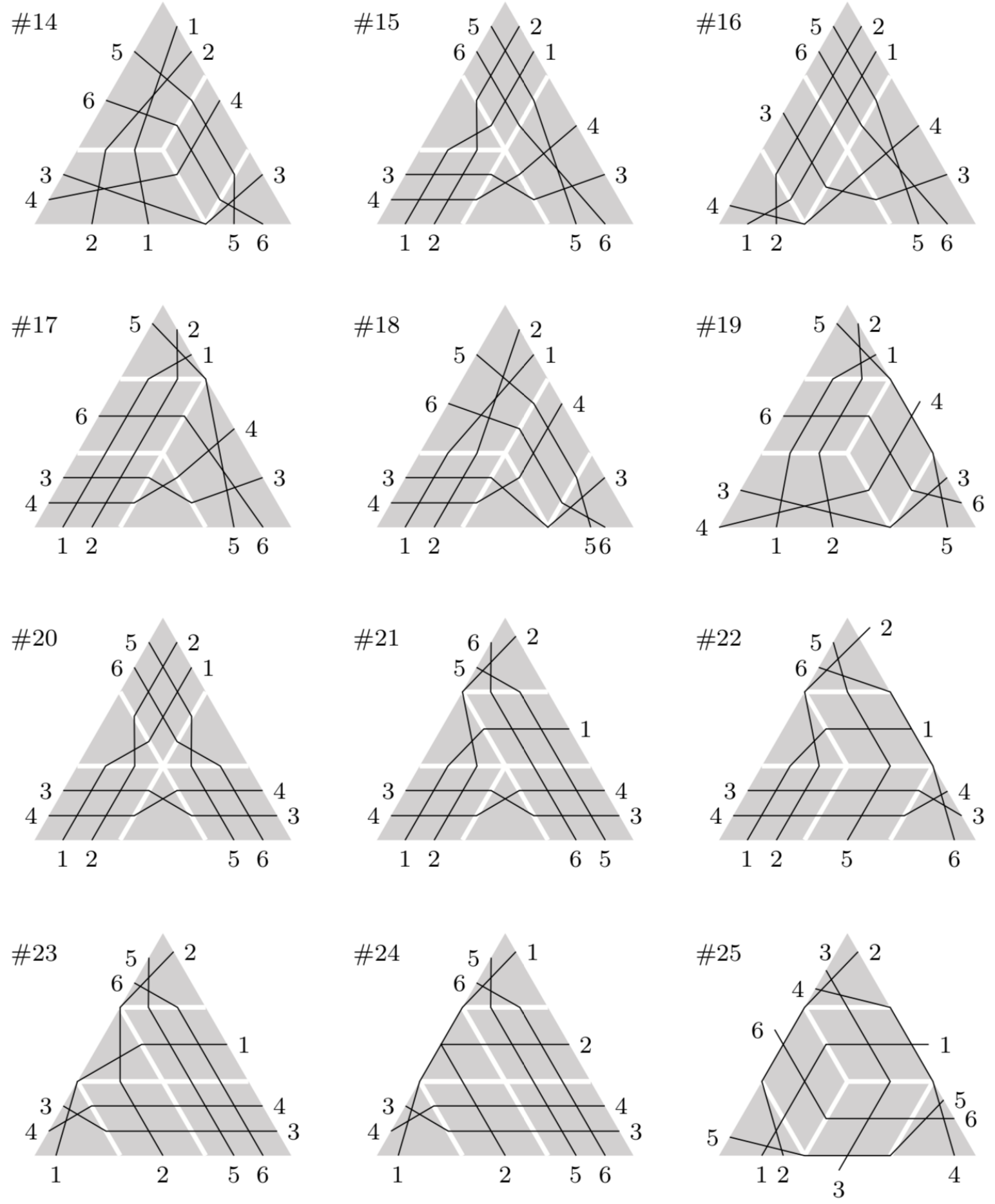

FiguRE 25. (From [Ale15, Figure 5.13] with permission of the author.) 


\section{REFERENCES}

[Ale08] Valery Alexeev. Weighted grassmannians and stable hyperplane arrangements. (2008): preprint arXiv:0806.0881.

[Ale15] Valery Alexeev. Moduli of weighted hyperplane arrangements. Edited by Gilberto Bini, Martí Lahoz, Emanuele Macrì and Paolo Stellari. Advanced Courses in Mathematics. CRM Barcelona. Birkhäuser/Springer, Basel, 2015.

[CHSW11] Dustin Cartwright, Mathias Häbich, Bernd Sturmfels, and Annette Werner. Mustafin varieties. Selecta Math. (N.S.) 17 (2011), no. 4, 757-793.

[CS10] Dustin Cartwright and Bernd Sturmfels. The Hilbert scheme of the diagonal in a product of projective spaces. Int. Math. Res. Not. IMRN 2010, no. 9, 1741-1771.

[CT20] Ana-Maria Castravet and Jenia Tevelev. Derived category of moduli of pointed curves. I. Algebr. Geom. 7 (2020), no. 6, 722-757.

[Fal01] Gerd Faltings. Toroidal resolutions for some matrix singularities. Moduli of abelian varieties (Texel Island, 1999), 157-184, Progr. Math., 195, Birkhäuser, Basel, 2001.

[FS05] Eva Maria Feichtner and Bernd Sturmfels. Matroid polytopes, nested sets and Bergman fans. Port. Math. (N.S.) 62 (2005), no. 4, 437-468.

[GKZ08] Izrail' M. Gel'fand, Mikhail M. Kapranov, and Andrei V. Zelevinsky. Discriminants, resultants and multidimensional determinants. Reprint of the 1994 edition. Modern Birkhäuser Classics. Birkhäuser Boston, Inc., Boston, MA, 2008.

[GM82] Izrail' M. Gel'fand and Robert D. MacPherson. Geometry in Grassmannians and a generalization of the dilogarithm. Adv. Math. 44 (1982), no. 3, 279-312.

[GP91] Lothar Gerritzen and Meinolf Piwek. Degeneration of point configurations in the projective plane. Indag. Math. (N.S.) 2 (1991), no. 1, 39-56.

[GG14] Noah Giansiracusa and William Danny Gillam. On Kapranov's description of $\overline{\mathrm{M}}_{0, n}$ as a Chow quotient. Turkish J. Math. 38 (2014), no. 4, 625-648.

[HKT06] Paul Hacking, Sean Keel, and Jenia Tevelev. Compactification of the moduli space of hyperplane arrangements. J. Algebraic Geom. 15 (2006), no. 4, 657-680.

[HKT09] Paul Hacking, Sean Keel, and Jenia Tevelev. Stable pair, tropical, and log canonical compact moduli of del Pezzo surfaces. Invent. Math. 178 (2009), no. 1, 173-227.

[HS04] Mark Haiman and Bernd Sturmfels. Multigraded Hilbert schemes. J. Algebraic Geom. 13 (2004), no. $4,725-769$.

[Has03] Brendan Hassett. Moduli spaces of weighted pointed stable curves. Adv. Math. 173 (2003), no. 2, 316-352.

[Kap93a] Mikhail M. Kapranov. Chow quotients of Grassmannians. I. I. M. Gel'fand Seminar, 29-110, Adv. Soviet Math., 16, Part 2, Amer. Math. Soc., Providence, RI, 1993.

[Kap93b] Mikhail M. Kapranov. Veronese curves and Grothendieck-Knudsen moduli space $\overline{\mathrm{M}}_{0, n}$. J. Algebraic Geom. 2 (1993), no. 2, 239-262.

[KSZ91] Mikhail M. Kapranov, Bernd Sturmfels, and Andrei V. Zelevinsky. Quotients of toric varieties. Math. Ann. 290 (1991), no. 4, 643-655.

[KT06] Sean Keel and Jenia Tevelev. Geometry of Chow quotients of Grassmannians. Duke Math. J. 134 (2006), no. 2, 259-311.

[Laf03] Laurent Lafforgue. Chirurgie des grassmanniennes. CRM Monograph Series, 19. American Mathematical Society, Providence, RI, 2003.

[LM00] Andrei S. Losev and Yurii Ivanovich Manin. New moduli spaces of pointed curves and pencils of flat connections. Michigan Math. J. 48 (2000), 443-472.

[Lux08] Mark A. Luxton. The log canonical compactification of the moduli space of six lines in $\mathbb{P}^{2} . \mathrm{PhD}$ dissertation, University of Texas at Austin (2008).

[Mol21] Samouil Molcho. Universal stacky semistable reduction. Israel J. Math. (2021). https://doi. org/10.1007/s11856-021-2118-0 
[Mus78] G. A. Mustafin. Non-archimedean uniformization. Mat. Sb. (N.S.) 105(147) (1978), no. 2, 207$237,287$.

[Oxl11] James Oxley. Matroid theory. Second edition. Oxford Graduate Texts in Mathematics, 21. Oxford University Press, Oxford, 2011.

[Ray72] Michel Raynaud. Flat modules in algebraic geometry. Compos. Math. 24 (1972), 11-31.

[SS04] David Speyer and Bernd Sturmfels. Tropical Grassmannian. Adv. Geom. 4 (2004), no. 3, 389-411.

[Tev07] Jenia Tevelev. Compactifications of subvarieties of tori. Amer. J. Math. 129 (2007), no. 4, 10871104.

[Wer01] Annette Werner. Compactification of the Bruhat-Tits building of PGL by lattices of smaller rank. Doc. Math. 6 (2001), 315-341.

Department of Mathematics, KTH Royal Institute of Technology, SE-100 44 Stockholm, SWEDEN

Email address: lucsch@math.kth.se

Department of Mathematics and Statistics, University of Massachusetts, Amherst, Ma 01003. Laboratory of Algebraic Geometry and its Applications, HSE, Moscow, Russia.

Email address: tevelev@math.umass.edu 